\title{
Dynamic neural representations in motor learning: Technical and empirical contributions
}

Citation for published version (APA):

Reithler, J. (2007). Dynamic neural representations in motor learning: Technical and empirical contributions. [Doctoral Thesis, Maastricht University]. Datawyse / Universitaire Pers Maastricht. https://doi.org/10.26481/dis.20071024jr

Document status and date:

Published: 01/01/2007

DOI:

10.26481/dis.20071024jr

Document Version:

Publisher's PDF, also known as Version of record

\section{Please check the document version of this publication:}

- A submitted manuscript is the version of the article upon submission and before peer-review. There can be important differences between the submitted version and the official published version of record.

People interested in the research are advised to contact the author for the final version of the publication, or visit the DOI to the publisher's website.

- The final author version and the galley proof are versions of the publication after peer review.

- The final published version features the final layout of the paper including the volume, issue and page numbers.

Link to publication

\footnotetext{
General rights rights.

- You may freely distribute the URL identifying the publication in the public portal. please follow below link for the End User Agreement:

www.umlib.nl/taverne-license

Take down policy

If you believe that this document breaches copyright please contact us at:

repository@maastrichtuniversity.nl

providing details and we will investigate your claim.
}

Copyright and moral rights for the publications made accessible in the public portal are retained by the authors and/or other copyright owners and it is a condition of accessing publications that users recognise and abide by the legal requirements associated with these

- Users may download and print one copy of any publication from the public portal for the purpose of private study or research.

- You may not further distribute the material or use it for any profit-making activity or commercial gain

If the publication is distributed under the terms of Article $25 \mathrm{fa}$ of the Dutch Copyright Act, indicated by the "Taverne" license above, 


\section{Dynamic neural representations in motor learning: \\ Technical and empirical contributions}

Joel Reithler 
Printed by Datawyse / Universitaire Pers Maastricht

Cover design M. Reithler / J. Reithler

Copyright $\quad$ (C) J. Reithler, Maastricht 2007

ISBN $\quad 978-90-5278-669-8$ 


\title{
Dynamic neural representations in motor learning: Technical and empirical contributions
}

\author{
Proefschrift \\ ter verkrijging van de graad van doctor aan de Universiteit Maastricht, \\ op gezag van de Rector Magnificus, prof. mr. G.P.M.F. Mols, \\ volgens het besluit van het College van Decanen, \\ in het openbaar te verdedigen \\ op woensdag 24 oktober 2007 om 14.00 uur \\ door \\ Joel Reithler
}

Geboren op 2 mei 1977 te Heerlen

$\bigcup_{\mathrm{M}}^{\mathrm{M}}$ 


\section{Promotor:}

Prof. dr. R. Goebel

\section{Copromotor:}

Dr. H.I. van Mier

\section{Beoordelingscommissie:}

Prof. dr. H. Hospers (voorzitter)

Prof. dr. F. di Salle

Prof. dr. G. Fink (Forschungszentrum Jülich, Germany)

Dr. E. Formisano

Dr. I. Toni (FC Donders Centre Nijmegen) 
To my parents 


\section{Table of contents}

Chapter 1 General introduction:

Exploring the cortical motor system p. 1

Chapter 2 Resistance-based high resolution recording of predefined 2-dimensional pen trajectories in an fMRI setting

Chapter 3 Dynamic neural representations of continuous motor sequences:

Effects of intra- and inter-session learning p. 39

Chapter 4 Nonvisual motor learning influences abstract action observation p. 69

$\begin{array}{lll}\text { Chapter } 5 \text { General discussion } & \text { p. } 93\end{array}$

$\begin{array}{ll}\text { Summary p. } 109 & \end{array}$

$\begin{array}{ll}\text { Samenvatting } & \text { p. } 113\end{array}$

$\begin{array}{ll}\text { Acknowledgements } & \text { p. } 117\end{array}$

$\begin{array}{ll}\text { Curriculum vitae } & \text { p. } 119\end{array}$ 
Chapter 1

General introduction:

Exploring the cortical motor system 
After hundreds of years during which mental functions were initially attributed to the heart and later to the ventricular cavities of the brain, the first publication on motor control in which the cerebral cortex was assigned a prominent role appeared in the second half of the $19^{\text {th }}$ century and was written by Gustav Fritsch and Edouard Hitzig (see Bennett \& Hacker, 2002). In their seminal work, they documented the occurrence of contralateral muscular contractions when specific superficially located parts of a dog's brain were electrically stimulated. About thirty years later (in 1902), Grunbaum and Sherrington unequivocally established the concept of a spatially circumscribed and somatotopically organized 'motor cortex' in primates, described in more detail for the human brain in 1950 by Penfield and Rasmussen. Although more recent studies on the somatotopic organization of the primary motor cortex have led to a re-evaluation of these earlier findings (Sanes \& Donoghue, 2000; Schieber, 2001), the work of these pioneers is of great importance because it constitutes one of the earliest demonstrations of a fundamental organizational principle of the brain, namely the principle of functional specialization. This defining feature reflects the idea that circumscribed brain regions or populations of neurons fulfill a particular functional role, and is still a central tenet of today's modern neuroscience (Friston, 2002).

Similar to the impact of these classic studies, the progress that was achieved on a technical level by the end of the $20^{\text {th }}$ century heralded a new era of research on the neural bases of motor control and mental functions in general. The development of micro-electrodes resulted in a rich literature based on neurophysiological studies in cats and monkeys, and the advent of sophisticated neuroimaging techniques such as Positron Emission Tomography (PET) and functional Magnetic Resonance Imaging (fMRI) provided the means to study the functional anatomy of the human brain in vivo. Because these techniques made it possible to link cognitive processes to neural events, they ultimately led to the synergy between cognitive psychology and neuroscience which we nowadays refer to as the field of cognitive neuroscience (Posner \& DiGirolamo, 2000).

The work which is presented in the current thesis represents a modest (but hopefully significant) fraction of this ongoing endeavor to unravel the inner workings of the human brain through the use of modern imaging techniques. Probably the most consistent finding which has been reported in the neuroimaging literature over the years has been the observation that almost any cognitive task is associated with increases in activation throughout a distributed network of brain areas, instead of being mediated by a single specialized region which works in complete isolation (Posner, 2003). So, although a given brain region might fulfill a particular function (as indicated by the classic studies cited earlier), several regions have to work together to meet the current task requirements and conjointly underlie the emergence of purposeful behavior. 
This principle of functional integration is therefore complementary to the organizational feature of functional specialization mentioned above, and has proven to be equally important (Friston, 2002).

Widespread networks of interacting brain regions have also been reported in the domains of motor planning and execution. Interestingly though, the identified networks appear to be dynamic in the sense that the involvement of the implicated brain regions can change with practice (van Mier, 2000). However, only few imaging studies have investigated the whole time course of learning (Doyon, Penhune, \& Ungerleider, 2003), and the present knowledge is largely based on sequence learning tasks requiring discrete finger tapping movements (Sanes, 2003). Conversely, the fMRI experiments which will be described in the following chapters focused on the learning of continuous movement sequences and spanned a time window of several days during which learning was monitored both on the behavioral and the neural level. An additional study was performed to gain further insights into how the stored information on the learned movement patterns can be accessed in a different context once practice has ended.

Because a large number of areas has generally been thought to be involved in the production and acquisition of motor routines, a short overview of the anatomically and/or functionally defined brain regions which constitute the studied central motor system will be given in the following section. In this way, the currently advocated parcellation scheme of cortical motor areas can be introduced and can function as a background for the chapters to follow. Since a substantial part of the work described in this thesis was concerned with changes in neural representations due to learning, this introductory chapter additionally contains a short section on the concept of neural plasticity. Furthermore, a concise description of the most prominent characteristics of fMRI will be presented to emphasize its unique capabilities but also point to the inherent technical challenges which needed to be faced in order to successfully conduct the reported experiments. Finally, a more detailed outline of the thesis' content will be presented.

\section{The neural correlates of motor control and learning}

The way in which the production and acquisition of skillful movements is intertwined with our daily activities can hardly be overstated. Our ability to translate intentions into actions is crucial in order to interact with the world that surrounds us, and basically all forms of communication depend on the production of meaningful actions in one way or another. The various movements we adaptively generate and the stored motor routines we apply are 
so embedded in our daily lives that we often only become aware of them in situations where we fail to execute them properly. In contrast to its evident ecological importance, research on motor control has only received little attention in psychology (for an essay regarding this issue, see Rosenbaum, 2005). Similarly, although motor paradigms have been used in fMRI since its inception, the relative number of neuroimaging studies devoted to the investigation of brain mechanisms in motor control has been rather limited in comparison to other domains (Illes, Kirschen, \& Gabrieli, 2003). One of the reasons why IMRI research in the motor domain has been lagging behind compared to studies on perception, emotion, or memory is probably related to the aforementioned technical challenges that accompany the implementation of motor tasks in an fMRI environment (Culham \& Valyear, 2006), a point which also had to be extensively addressed in the context of the present research. Nevertheless, considerable progress has been made in the last decades due to the application of neuroimaging (Mattay \& Weinberger, 1999; Culham \& Valyear, 2006), as well as microanatomical mapping and neurophysiological techniques (Rizzolatti \& Luppino, 2001). Next, a brief introduction on the central motor system and its features will be provided which reflects these fairly recent advances. Because of their prominent role in the ensuing chapters and for the sake of brevity, we will focus on the implicated cortical areas, although it should be noted that subcortical structures such as the basal ganglia and the cerebellum are undoubtedly involved in the production of voluntary movements.

\subsection{Organization of the cortical motor system}

As mentioned earlier, the insight that the cortical sheet of the brain should be the main locus under investigation when trying to elucidate the bases of a variety of behavioral phenomena can be seen as a relatively recent development from a historical point of view. Studies on the functional neuroanatomy of the motor system initially focused on the primary motor cortex (M1) because its excitation led to muscle responses which were easily observable. A striking outcome of these experiments was that varying the site of stimulation resulted in muscle contractions in different parts of the body. This form of somatotopic representation was later confirmed in numerous neuroimaging studies (e.g., Grafton et al., 1991; Lotze et al., 2000), although more recent evidence shows that whereas the different limbs can be dissociated, the within-limb somatotopy might be neither spatially discrete nor sequentially ordered (Schieber, 2001). Because the primary motor cortex is the brain's greatest source of output to the spinal cord, it is often regarded as representing the lowest level of the central motor hierarchy. This, however, does not necessarily imply that it merely functions as a simplistic relay station (Sanes \& Donoghue, 2000). Quite to the 
contrary, the information which is embedded in M1's activity patterns during the production of voluntary movements turns out to be fairly complex. One of the most salient features of M1 neurons is that they display directional selectivity, meaning that a particular neuron preferentially discharges when a movement is made in a certain direction. By pooling the information which is conveyed by a whole population of neurons, a so-called 'population vector' can be calculated which provides an estimate of the neurally coded direction that closely matches the actual movement direction (Georgopoulos, Schwartz, \& Kettner, 1986). In fact, this decoding can be performed with such a high precision that it enables otherwise paralyzed patients to move a cursor just by their thoughts via intracranial recordings (Hochberg et al., 2006). However, the activity patterns of individual M1 neurons additionally code other types of information (such as movement velocity, applied force and muscular activity). Consequently, the exact nature of M1 representations is still part of an ongoing debate (Scott, 2003).

Complementary to advances in the field of cytoarchitectonics and the results obtained in neurophysiological studies in the monkey, modern neuroimaging research has contributed to the delineation of additional regions (besides M1), which constitute the higher levels of the motor hierarchy (e.g., Fink et al., 1997). Most prominent among them are the 'premotor' areas (a term introduced by Fulton, 1935) on the lateral surface of the hemispheres. Initially, the famous cytoarchitectonic studies by Brodmann (1909) suggested that the premotor cortex formed a single homogeneous cortical region (Brodmann Area (BA) 6), right in front of the primary motor cortex (BA 4). However, converging evidence from several disciplines has shown that the lateral premotor cortex consists of at least two distinct regions (Geyer et al., 2000), both of which can be subdivided into two different sectors (Rizzolatti, Fogassi, \& Gallese, 2002). In the macaque, two distinct regions (F2 and F7) have been identified based on cytoarchitectural and histochemical criteria, which together form what has been referred to as the 'dorsal' premotor cortex (PMd). In the same fashion, monkey areas F4 and F5 conjointly constitute the 'ventral' premotor cortex (PMv). A similar parcellation scheme has been proposed for the human premotor cortex (Rizzolatti, Luppino, \& Matelli, 1998), where the dorsal part of the precentral gyrus and the caudal part of the frontal gyrus correspond to areas F2 and F7, and the ventral part of the precentral gyrus together with the more anteriorly located BA 44 form the human homologues of areas F4 and F5. To illustrate the relative position of each of these regions, the described parcellation scheme is depicted in Figure 1 (on the left). However, it should be noted that the precise location and the exact delineation of these regions is still under debate (Picard $\&$ Strick, 2001). With respect to their functional roles, several observations have been made. Most importantly, these areas show anticipatory responses before any movement is executed (in contrast to M1) and have therefore been 
implicated in the selection and preparation of upcoming movements (e.g., Toni, Thoenissen, \& Zilles, 2001; Cisek \& Kalaska, 2005). However, recent studies have indicated that the premotor cortex might also be involved in more cognitive aspects of behavior which are not directly coupled to any overt motor responses, such as action recognition (Rizzolatti, Fogassi, \& Gallese, 2002) or the extraction of sequential information (Schubotz \& von Cramon, 2003). In addition, attempts have been made to further characterize the specific role of the aforementioned subregions in the production of voluntary movements. For example, based on data from a visually guided reaching task in which the instruction on where to move and the instruction on which arm to use were temporally separated, Hoshi \& Tanji (2004a) suggested that the PMv mainly represents information related to the cue and target locations, whereas this information is combined with the instructions on which effectors to use in the PMd. In accordance with this hypothesis, the PMd was recently shown to use a 'relative position' code that specifies the difference between the position of the eyes, the hand, and the target to be reached (Pesaran, Nelson, \& Andersen, 2006). Another dissociation which has been proposed is related to the differences between the rostrally and caudally located subregions within PMd and PMv. Premotor neurons with stronger sensory properties are more frequently found in the rostral subregions, and compared to the caudal zones these subregions are also anatomically more tightly connected to the prefrontal than to the primary motor cortex (Schubotz \& von Cramon, 2003).

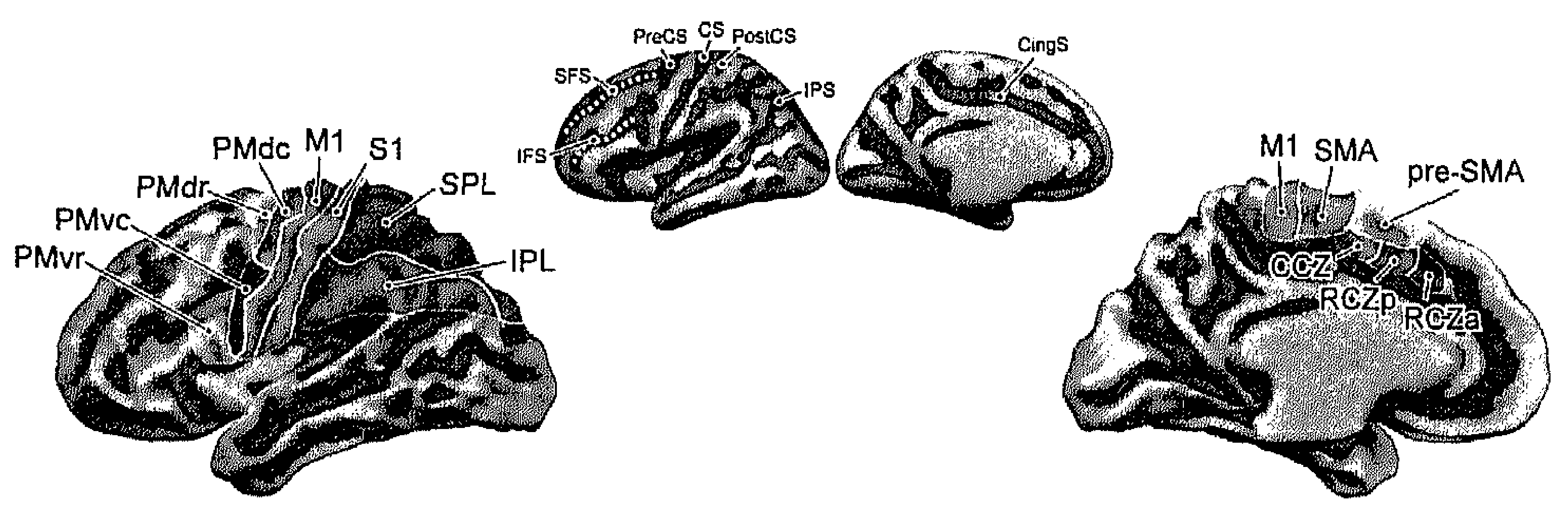

Figure 1. Schematic overview of the cortical areas involved in motor control and learning, projected onto a mesh reconstruction of the left hemisphere of the human brain. The folded representations illustrate the relative positions of the microanatomically and functionally defined regions mentioned in the text. On the left, the lateral view is depicted, whereas the right part of the figure shows the medial view. The smaller inflated representations in the middle are used to indicate the most relevant macroanatomical landmarks. The concave sulci are shown in dark grey; the convex gyri are shown in light grey. Used abbreviations: M1, primary motor cortex; S1, primary somatosensory cortex; PMd, dorsal premotor cortex; PMdc, caudal part of PMd; PMdr, rostral part of PMd; PMv, ventral premotor cortex; PMvc, caudal part of PMv; PMvr, rostral part of PMv; SPL, superior parietal lobule; IPL, inferior parietal lobule; CS, central sulcus; PreCS, precentral sulcus; SFS, superior frontal sulcus; IFS, inferior frontal sulcus; PostCS, postcentral sulcus; IPS, intraparietal sulcus; CingS, cingulate sulcus; SMA, supplementary motor area; pre- 
Chapter 1

SMA, pre-supplementary motor area; CCZ, caudal cingulate zone; $R C Z p$, posterior part of rostral cingulate zone; $\mathrm{RCZa}$, anterior part of rostral cingulate zone.

A comparable picture has emerged with respect to the premotor cortex on the medial surface of the brain's hemispheres. Although previous cytoarchitectonic studies showed that the mesial frontal cortex lying rostral to the primary motor cortex consisted of two separate regions (defined as areas $6 \mathrm{a} \alpha$ and $6 \mathrm{a} \beta$ by Vogt \& Vogt, 1919), this distinction was considered as being functionally insignificant based on the findings from stimulation studies performed in the 1950s (Woolsey et al., 1952). Accordingly, the mesial part of the premotor cortex was believed to consist of a single region which was termed the 'supplementary' motor area (SMA). However, there now is a widely held consensus that the medial wall contains at least two distinct areas in both monkeys and humans: the 'pre-SMA' and the 'SMA proper' (Tanji, 1996). These more general labels are suggested to correspond to cytoarchitectonic areas F6 and F3 in monkeys, and areas $6 \mathrm{a} \alpha$ and $6 \mathrm{a} \beta$ in humans, respectively ${ }^{1}$. Moreover, this distinction is supported by imaging studies in humans which showed differences between the pre-SMA and SMA proper with respect to their connectivity patterns (e.g., Johansen-Berg et al., 2004) and their functional properties (e.g., Sakai et al., 1999). Activation in the pre-SMA is associated with the establishment and retrieval of modality- and effector-independent visuo-motor associations and the visually instructed preparation of movements, whereas the SMA displays activations which are more strictly related to movement execution per se (Picard \& Strick, 2001; Hoshi \& Tanji, 2004b). But the heterogeneity of the medial cortex does not stop there. Again based on work in the monkey, three additional motor areas appear to exist along the cingulate sulcus, which are collectively called the 'cingulate motor areas' (Picard \& Strick, 1996; 2001). In humans, these cingulate motor areas include the 'rostral cingulate zone' (RCZ), which is subdivided in an anterior and a posterior part, and the 'caudal cingulate zone' or $\mathrm{CCZ}$ (respectively corresponding to the rostral (CMAr), ventral (CMAv) and dorsal (CMAd) cingulate motor areas in the monkey). Whereas the $\mathrm{CCZ}$ is predominantly involved in movement execution, the RCZ has been implicated in both conflict monitoring and the selection of actions (Picard \& Strick, 2001). The approximate locations of the medial motor areas are shown in Figure 1 (on the right).

The final building blocks of the cortical motor system can be found in the posterior parietal cortex (PPC; Fogassi \& Luppino, 2005). The anterior border of the PPC is formed by the somatosensory cortex on the postcentral gyrus, and its posterior border coincides with the transition into the occipital lobe (Culham

\footnotetext{
${ }^{1}$ Actually, in humans an even greater anatomical fractionation has been suggested by Vorobiev et al. (1998), who showed that the 'SMA proper' can be subdivided in a caudal and a rostral sector.
} 
\& Kanwisher, 2001). The PPC is divided into the superior parietal lobule (SPL) and the inferior parietal lobule (IPL) by the intraparietal sulcus (IPS). Because the PPC is anatomically situated between areas which are involved in the processing of somatosensory information on the one hand, and visual information on the other, it was traditionally seen as a general 'association' area involved in the integration of information from the different sensory modalities. However, numerous studies have meanwhile provided evidence for the view that the PPC comprises a "mosaic of specialized areas" (Culham \& Valyear, 2006 , p. 205), which all have a particular function in the sensory control of action. Specializations have been proposed with regard to certain types of movements (e.g., reaching vs. grasping) and the reference frames (e.g., hand vs. eye-centered) in which movements with different effectors can be planned and executed (Andersen \& Buneo, 2002; Culham, Cavina-Pratesi, \& Singhal, 2006). Importantly, the identified parietal subregions are reciprocally connected to the motor areas in the frontal lobe. These connections constitute a collection of specialized circuits that function in parallel to each other (Rizzolatti \& Luppino, 2001) and seem to underlie the transformation from internally generated as well as externally guided intentions to skillfully produced actions.

\subsection{Plasticity and Learning}

As the preceding overview has shown, a widely distributed cortical network comprising a large number of distinct areas is involved in the generation of willed actions. On top of this complex arrangement, it has become increasingly clear over the last decades that cortical representations and their underlying neural architectures are not static constructs, but inherently hold the potential to dynamically adapt to new situations and altered requirements throughout life (Buonomano \& Merzenich, 1998). This neural plasticity can be observed in relation to learning (Kelly \& Garavan, 2005) as well as during the recovery from injury (Ward, 2005), and was suggested to represent "evolution's invention to enable the nervous system to escape the restrictions of its own genome" (Pascual-Leone et al., 2005, p. 377). One of the first and most striking examples of cortical reorganization was described by Merzenich and colleagues (1983), who showed that the ablation of the nerve which signals sensory information from the digits to the brain resulted in a dramatic change in the layout of the somatotopic map in the primate somatosensory cortex. Instead of leaving a patch of cortex completely unresponsive, the vacant space was progressively occupied by neighboring representations of other parts of the hand whose efferent connections were left intact. Cortical plasticity is not limited to cases in which the brain is deprived of its normal input, but can also be induced by behavioral training. Interestingly, additional experiments focusing on the primate somatosensory cortex showed that the cortical representations of the 
Chapter 1

body parts involved in training did not only expand over the course of learning, but their expansion was accompanied by a reduction in receptive field size of the implicated neurons (Jenkins et al., 1990). Because smaller receptive fields point to higher spatial resolution capabilities, these findings indicated that the observed changes also had direct functional implications. In humans, similar observations were made. For example, the somatosensory representation of the index finger used for reading Braille manuscripts was found to be significantly larger in Braille readers compared to both that of the index finger of the nondominant hand as well as that of the matching index finger in non-Braille reading control subjects (Pascual-Leone \& Torres, 1993). Later, evidence for experience-specific extensions of cortical representations was also reported for the primary motor cortex, both in monkeys (e.g., Nudo et al., 1996) and in humans (e.g., following extensive training of a finger-to-thumb opposition sequence; Karni et al., 1995).

However, further neuroimaging studies on neural activation changes in motor learning in which the whole brain (i.e., not only M1) was covered, have generally led to a more complicated pattern of results. Next to increases and decreases in regional activations (where expansions might be observed as increases in activation strength due to an insufficient spatial resolution or applied spatial smoothing; Poldrack, 2000), complete reorganizations within or extensions beyond the network of initially involved regions have been reported (van Mier, 2000). These discrepancies can probably be best explained by differences in the type of task used (e.g., sequence vs. adaptation learning), the specific movements which were required (e.g., discrete button presses vs. continuous tracking), the way in which learning took place (e.g., massed vs. distributed practice or implicit vs. explicit learning instructions), the amount of practice involved (e.g., minutes vs. weeks), and the time points at which imaging data were acquired (e.g., a single vs. multiple scanning sessions) ${ }^{2}$. Despite a plethora of contributing variables and the divergence in the obtained results, recent efforts have been directed towards the specification of models which try to reconcile the apparently mutually exclusive findings on neural changes accompanying the acquisition of a novel skill (Doyon \& Benali, 2005; Kelly \& Garavan, 2005; Ashe et al., 2006). Although still constituting an open issue, resolving these incompatibilities is important to our understanding of the very nature of cortical processing per se, and will help in refining the methods which can be applied in the field of neurorehabilitation (Robertson \& Murre, 1999; Krakauer, 2006).

\footnotetext{
${ }^{2}$ In addition, the already fuzzy picture which has emerged is further complicated by findings related to socalled offline learning effects (spontaneous improvements which can be observed after practice has ended; e.g., Cohen et al., 2005) and the role of sleep in the consolidation of what has been previously learned (Walker \& Stickgold, 2006).
} 


\section{Characteristics of functional Magnetic Resonance Imaging}

The experiments which are presented in the subsequent chapters were all performed using fMRI. In order to substantiate the choice to use this particular neuroimaging method, its main characteristics will be presented here together with some of its inherent drawbacks.

Neuroimaging techniques which are based on the principle of magnetic resonance have revolutionized the way in which research on human brain functions can be conducted and have rapidly advanced from the moment the first MR images were published by Paul Lauterbur in 1973. Most importantly, fMRI enables the measurement of human brain activity in vivo, basically meaning that it is possible to witness how neural events unfold while subjects are actively engaged in a given task. Even though Electroencephalography (EEG) and Magnetoencephalography (MEG) provide more detailed information in the temporal domain, fMRI is superior to all other commonly used neuroimaging techniques with respect to the spatial detail which can be achieved. For example, in the studies which will be described in the following chapters, about 86,000 volume elements (voxels) were measured every 1.25 seconds and conjointly formed an imaging volume which covered the subjects' entire brain. The fact that data from all parts of the brain can be simultaneously collected also shows that fMRI nicely complements neurophysiological recordings using micro-electrodes in non-human primates, which are mostly restricted to a single cortical recording site. Furthermore, fMRI is a noninvasive method because its use does not require the injection of any contrast agents (in contrast to PET). This property is especially important in the context of studies related to learning phenomena, because it means that the same subject can be repeatedly scanned within a short period of time without imposing any health risks.

It should be noted that $\mathrm{AMRI}$ only provides an indirect measure of neural activity. As a particular population of neurons starts to discharge more vigorously while the subject is performing a certain task, more oxygenated blood is supplied to this activated brain region in order to meet the increased metabolic requirements. The measured fMRI signal reflects this vascular response and consequently constitutes a delayed as well as a spatially and temporally smoothed counterpart of the underlying neural activity. Therefore, the upper limits regarding the temporal and spatial resolution which can be achieved will ultimately be dictated by biology, not technology (Bandettini, Birn, \& Donahue, 2000). Even though the fMRI signal only grants an indirect way to monitor the brain's activity via the hemodynamic response, it has been shown to be directly coupled to the underlying neuronal activity based on 


\section{Chapter 1}

simultaneously acquired neurophysiological and fMRI data in monkeys (Logothetis, 2003).

One of the downsides of fMRI is that the measurements are performed within a strong static magnetic field (ranging from $1.5 \mathrm{~T}$ to $9 \mathrm{~T}$ for different scanners, which is about 30 to 180 thousand times stronger than the earth's magnetic field). This poses severe constraints on any additional equipment one would want to use because all of its components have to be fabricated using nonmagnetic materials and have to function properly in such an extraordinary environment. Likewise, one has to ensure that the measurement of the relatively small evoked responses which are detected by fMRI (increases in magnitude in the range of $0.5-3 \%$ with respect to a resting baseline) is not negatively affected by the use of any complementary recording equipment through electromagnetic radiation. Furthermore, there are additional drawbacks which are specifically related to $\mathrm{fMRI}$ research on motor control. First, the confined space of the MR scanner's bore generally does not leave much room to freely execute a wide range of upper limb movements and therefore limits the types of movements which can be studied. Secondly, extensive movements are almost inevitably accompanied by changes in the position of the subject's head, which can have detrimental effects on the measured MR signal and can lead to wrong inferences at the analysis stage because the head motion is correlated to the experimental protocol. Finally, performing the movement per se can have a negative influence on the quality of the imaging data, because it introduces transient local inhomogeneities in the magnetic field which cannot be easily detected or compensated. Nevertheless, fMRI enables us to catch a unique glimpse of what happens within the 'black box' the human brain has constituted for centuries, and provides us with a wealth of information which cannot be obtained by any other method to date.

\section{Outline of the thesis}

As mentioned above, the research which is described in the current thesis mainly focused on the neural basis of motor sequence learning, the way in which brain activation patterns change as learning progresses, and the possibilities of accessing information on the stored motor routines once practice has ended.

In contrast to the vast majority of fMRI studies in the motor domain, the sequences which had to be learned did not consist of discrete button presses in response to visually or auditorily presented stimuli, but formed continuous movement trajectories which had to be traced without any form of visual or auditory feedback or guidance. The trajectory which formed the sequence to be 
learned was determined by the segments of a predefined maze design (van Mier et al., 1998). Subjects could only make use of the proprioceptive information they could extract while actually performing the pen tracing movements through the maze's groove and the additional somatosensory cues they could derive along the way.

However, in order to simultaneously monitor the behavioral and neural manifestations of learning, a setup needed to be developed which allowed the recording of the performed pen tracing movements in an fMRI setting. The technical issues which needed to be considered and the methods which were applied to accomplish a detailed tracking are described in Chapter 2. This chapter gives an overview of the setup's components (including the custombuilt hardware and the in-house written data acquisition, calibration and analysis software), and describes the principles of the applied recording method. Furthermore, the ways in which the setup's validation and MR-compatibility were established are reported.

The study described in Chapter 3 was designed to gain further insights into the neural correlates of the production and acquisition of continuous motor sequences. Most importantly, the aim was to capture the dynamic nature of this process by showing how the neural representations of the acquired movement sequences might change over the course of learning. The behavioral and neural changes due to practice were monitored across two different time scales (from a few minutes to several days) by including two separate scanning sessions and an extended practice condition in which one of the sequences was trained during five consecutive days.

The main research question of the study reported in Chapter 4 was whether the brain regions which were involved in learning to perform a given motor sequence, would also be recruited if information on the executed sequence needed to be accessed in a different context. In this experiment, subjects were confronted with a visually presented trajectory and were asked to identify the trajectories which matched the previously performed but unseen motor sequence. The results are discussed in the light of an internal simulation process which is supposed to underlie the recognition and understanding of observed actions performed by others.

Finally, the findings of the presented studies will be summarized in Chapter 5, which also includes a discussion on the implications of their outcome and the way future research on these topics could be envisioned. 


\section{Chapter 1}

\section{References}

Andersen, R.A., \& Buneo, C.A. (2002). Intentional maps in posterior parietal cortex. Annual Review of Neuroscience, 25, 189-220.

Ashe, J., Lungu, O.V., Basford, A.T., \& Lu, X. (2006). Cortical control of motor sequences. Current Opinion in Neurobiology, 16, 213-221.

Bandettini, P.A., Birn, R.M., \& Donahue, K.M. (2000). Functional MRI: Background, methodology, limits, and implementation. In J.T. Cacioppo, L.G. Tassinary, \& G.G. Berntson (Eds.), Handbook of Psychophysiology (pp. 978-1014), Cambridge: University Press.

Bennett, M.R., \& Hacker, P.M.S. (2002). The motor system in neuroscience: A history and analysis of conceptual developments. Progress in Neurobiology, 67, 1-52.

Brodmann, K. (1909). Vergleichende Lokalisationslehre der Großhirnrinde. Barth: Leipzig.

Buonomano, D.V., \& Merzenich, M.M. (1998). Cortical plasticity: From synapses to maps. Annual Review of Neuroscience, 21, 149-186.

Cisek, P., \& Kalaska, J.F. (2005). Neural correlates of reaching decisions in dorsal premotor cortex: specification of multiple direction choices and final selection of action. Neuron, 45, 801-814.

Cohen, D.A., Pascual-Leone, A., Press, D.Z., \& Robertson, E.M. (2005). Off-line learning of motor skill memory: A double dissociation of goal and movement. Proceedings of the National Academy of Sciences USA, 102, 18237-18241.

Culham, J.C., \& Kanwisher, N.G. (2001). Neuroimaging of cognitive functions in human parietal cortex. Current Opinion in Neurobiology, 11, 157-163.

Culham, J.C., Cavina-Pratesi, C., \& Singhal, A. (2006). The role of parietal cortex in visuomotor control: What have we learned from neuroimaging? Neuropsychologia, 44, 2668-2684.

Culham, J.C., \& Valyear, K.F. (2006). Human parietal cortex in action. Current Opinion in Neurobiology, 16, 205-212.

Doyon, J., Penhune, V., \& Ungerleider, L.G. (2003). Distinct contribution of the cortico-striatal and cortico-cerebellar systems to motor skill learning. Neuropsychologia, 41, 252-262.

Doyon, J., \& Benali, H. (2005). Reorganization and plasticity in the adult brain during learning of motor skills. Current Opinion in Neurobiology, 15, 161-167. 
Fink, G.R., Frackowiak, R.S.J., Pietrzyk, U., \& Passingham, R.E. (1997). Multiple nonprimary motor areas in the human cortex. Journal of Neurophysiology, 77, 21642174.

Fogassi, L., \& Luppino, G. (2005). Motor functions of the parietal lobe. Current Opinion in Neurobiology, 15, 626-631.

Friston, K. (2002). Beyond phrenology: What can neuroimaging tell us about distributed circuitry? Annual Review of Neuroscience, 25, 221-250.

Fulton, J.F. (1935). A note on the definition of the "motor" and "premotor" areas. Brain, 58, 311-316.

Georgopoulos, A.P., Schwartz, A.B., \& Kettner, R.E. (1986). Neuronal population coding of movement direction. Science, 233, 1416-1419.

Geyer, S., Matelli, M., Luppino, G., \& Zilles, K. (2000). Functional neuroanatomy of the primate isocortical motor system. Anatomy and Embryology, 202, 443-474.

Grafton, S.T., Woods, R.P., Mazziotta, J.C., \& Phelps, M.E. (1991). Somatotopic mapping of the primary motor cortex in humans: Activation studies with cerebral blood flow and positron emission tomography. Journal of Neurophysiology, 66, 735-743.

Grunbaum, A.S.F., \& Sherrington, C.S. (1902). Observations on the physiology of the cerebral cortex of some of the higher apes (preliminary communication). Proceedings of the Royal Society of London, 69, 206-209.

Hochberg, L.R., Serruya, M.D., Friehs, G.M., Mukand, J.A., Saleh, M., Caplan, A.H., Branner, A., Chen, D., Penn, R.D., \& Donoghue, J.P. (2006). Neuronal ensemble control of prosthetic devices by a human with tetraplegia. Nature, 442, 164-171.

Hoshi, E., \& Tanji, J. (2004a). Functional specialization in dorsal and ventral premotor areas. Progress in Brain Research, 143, 507-511.

Hoshi, E., \& Tanji, J. (2004b). Differential roles of neuronal activity in the supplementary and presupplementary motor areas: From information retrieval to motor planning and execution. Journal of Neurophysiology, 92, 3482-3499.

Illes, J., Kirschen, M.P., \& Gabrieli, J.D.E. (2003). From neuroimaging to neuroethics. Nature Neuroscience, 6, 205.

Jenkins, W.M., Merzenich, M.M., Ochs, M.T., Allard, T., \& Guic-Robles, E. (1990). Functional reorganization of primary somatosensory cortex in adult owl monkeys after behaviorally controlled tactile stimulation. Journal of Neurophysiology, 63, 82-104. 


\section{Chapter I}

Johansen-Berg, H., Behrens, T.E.J., Robson, M.D., Drobnjak, I., Rushworth, M.F.S., Brady, J.M., Smith, S.M., Higham, D.J., \& Matthews, P.M. (2004). Changes in connectivity profiles define functionally distinct regions in human medial frontal cortex. Proceedings of the National Academy of Sciences USA, 101, 13335-13340.

Karni, A., Meyer, G., Jezzard, P., Adams, M.M., Turner, R., \& Ungerleider, L.G. (1995). Functional MRI evidence for adult motor cortex plasticity during motor skill learning. Nature, 377, 155-158.

Kelly, A.M.C., \& Garavan, H. (2005). Human functional neuroimaging of brain changes associated with practice. Cerebral Cortex, 15, 1089-1102.

Krakauer, J.W. (2006). Motor learning: Its relevance to stroke recovery and neurorehabilitation. Current Opinion in Neurology, 19, 84-90.

Lauterbur, P.C. (1973). Image formation by induced local interactions: Examples employing nuclear magnetic resonance. Nature, 242, 190-191.

Logothetis, N.K. (2003). The underpinnings of the BOLD functional magnetic resonance imaging signal. The Journal of Neuroscience, 23, 3963-3971.

Lotze, M., Erb, M., Flor, H., Huelsmann, E., Godde, B., \& Grodd, W. (2000). fMRI evaluation of somatotopic representation in human primary cortex. NeuroImage, 11, 473-481.

Mattay, V.S., \& Weinberger, D.R. (1999). Organization of the human motor system as studied by functional magnetic resonance imaging. European Journal of Radiology, 30, 105-114.

Merzenich, M.M., Kaas, J.H., Wall, J., Nelson, R.J., Sur, M., \& Felleman, D. (1983). Topographic reorganization of somatosensory cortical areas $3 \mathrm{~b}$ and 1 in adult monkeys following restricted deafferentation. Neuroscience, 8, 33-55.

Nudo, R.J., Milliken, G.W., Jenkins, W.M., \& Merzenich, M.M. (1996). Use-dependent alterations of movement representations in primary motor cortex of adult squirrel monkeys. The Journal of Neuroscience, 16, 785-807.

Pascual-Leone, A., \& Torres, F. (1993). Plasticity of the sensorimotor cortex representation of the reading finger in Braille readers. Brain, 116, 39-52.

Pascual-Leone, A., Amedi, A., Fregni, F., \& Merabet, L.B. (2005). The plastic human brain. Annual Review of Neuroscience, 28, 377-401.

Penfield, W., \& Rasmussen, T. (1950). The cerebral cortex of man: A clinical study of localization of function. New York: Hafner. 
Pesaran, B., Nelson, M.J., \& Andersen, R.A. (2006). Dorsal premotor neurons encode the relative position of the hand, eye, and goal during reach planning. Neuron, 51, 125134.

Picard, N., \& Strick, P.L. (1996). Motor areas of the medial wall: A review of their location and functional activation. Cerebral Cortex, 6, 342-353.

Picard, N., \& Strick, P.L. (2001). Imaging the premotor areas. Current Opinion in Neurobiology, 11, 663-672.

Poldrack, R.A. (2000). Imaging brain plasticity: conceptual and methodological issues a theoretical review. Neurolmage, 12, 1-13.

Posner, M.I., \& DiGirolamo, G.J. (2000). Cognitive Neuroscience: Origins and promise. Psychological Bulletin, 126, 873-889.

Posner, M.I. (2003). Imaging a science of mind. Trends in Cognitive Sciences, 7, 450453.

Rizzolatti, G., Luppino, G., \& Matelli, M. (1998). The organization of the cortical motor system: New concepts. Electroencephalography and Clinical Neurophysiology, 106, 283-296.

Rizzolatti, G., \& Luppino, G. (2001). The cortical motor system. Neuron, 31, 889-901.

Rizzolatti, G., Fogassi, L., \& Gallese, V. (2002). Motor and cognitive functions of the ventral premotor cortex. Current Opinion in Neurobiology, 12, 149-154.

Robertson, I.H., \& Murre, J.M.J. (1999). Rehabilitation of brain damage: Brain plasticity and principles of guided recovery. Psychological Bulletin, 125, 544-575.

Rosenbaum, D.A. (2005). The Cinderella of psychology: The neglect of motor control in the science of mental life and behavior. American Psychologist, 60, 308-317.

Sakai, K., Hikosaka, O., Miyauchi, S., Sasaki, Y., Fujimaki, N., \& Pütz, B. (1999). Presupplementary motor area activation during sequence learning reflects visuo-motor association. Journal of Neuroscience, 19, 1-6.

Sanes, J.N., \& Donoghue, J.P. (2000). Plasticity and primary motor cortex. Annual Review of Neuroscience, 23, 393-415.

Sanes, J.N. (2003). Neocortical mechanisms in motor learning. Current Opinion in Neurobiology, 13, 225-231.

Schieber, M.H. (2001). Constraints on somatotopic organization in the primary motor cortex. Journal of Neurophysiology, 86, 2125-2143. 
Chapter 1

Schubotz, R.I., \& von Cramon, D.Y. (2003). Functional-anatomical concepts of human premotor cortex: evidence from fMRI and PET studies. Neurolmage, 20, 120-131.

Scott, S.H. (2003). The role of primary motor cortex in goal-directed movements: insights from neurophysiological studies on non-human primates. Current Opinion in Neurobiology, 13, 671-677.

Tanji, J. (1996). New concepts of the supplementary motor area. Current Opinion in Neurobiology, 6, 782-787.

Toni, I., Thoenissen, D., \& Zilles, K. (2001). Movement preparation and motor intention. Neurolmage, 14, 110-117.

van Mier, H., Tempel, L.W., Perlmutter, J.S., Raichle, M.E., \& Petersen, S.E. (1998). Changes in brain activity during motor learning measured with PET: effects of hand of performance and practice. Journal of Neurophysiology, 80, 2177-2199.

van Mier, H. (2000). Human Learning. In A.W. Toga, \& J.C. Mazziotta (Eds.), Brain Mapping: The Systems (pp. 605-617), San Diego: Academic Press.

Vogt, O., \& Vogt, C. (1919). Ergebnisse unserer Hirnforschung. Journal für Psychologie und Neurologie, 25, 277-462.

Vorobiev, V., Govoni, P., Rizzolatti, G., Matelli, M., \& Luppino, G. (1998). Parcellation of human mesial area 6: Cytoarchitectonic evidence for three separate areas. European Journal of Neuroscience, 10, 2199-2203.

Walker, M.P., \& Stickgold, R. (2006). Sleep, memory, and plasticity. Annual Review of Psychology, 57, 139-166.

Ward, N.S. (2005). Neural plasticity and recovery of function. Progress in Brain Research, 150, 527-535.

Woolsey, C.N., Settlage, P.H., Meyer, D.R., Sencer, W., Pinto, H.T., \& Travis, A.M. (1952). Patterns of localization in precentral and "supplementary" motor areas and their relation to the concept of a premotor area. Research publications - Association for Research in Nervous and Mental Disease, 30, 238-264. 


\section{Chapter 2}

\section{Resistance-based high resolution recording of predefined 2-dimensional pen trajectories in an fMRI setting}

Based on: Reithler, J., Reithler, H., van den Boogert, E., Goebel, R., \& van Mier, H. (2006). Resistance-based high resolution recording of predefined 2dimensional pen trajectories in an fMRI setting. Journal of Neuroscience Methods, 152, 10-17. 


\begin{abstract}
The recent advent of functional Magnetic Resonance Imaging (fMRI) as a readily accessible neuroimaging method has led to exciting new insights into the functioning of the human motor system. However, technical complications related to the fMRI scanner environment often limit the ability to measure the desired behavioral data reflecting the subjects' movements. In order to perform kinematic registrations of predefined complex two-dimensional movement patterns while scanning, a new MR-compatible setup has been developed. The method presented here allows the recording of detailed pen tracing data during concurrent functional image acquisition. Essentially, temporally highly resolved resistance measurements are used to keep track of the covered distance across time, as applied here to the tracing of various mazes. In this way, the current setup adds the close monitoring of continuous tracing movements to the spectrum of behavioral data which can be successfully obtained in an fMRI setting.
\end{abstract}




\section{Introduction}

As has been commonly acknowledged, functional Magnetic Resonance Imaging (fMRI) has become a powerful and widely used tool to study the functional organization of the brain (e.g. Menon, 2001; Savoy, 2001). When focusing on our understanding of the human motor system, its application has resulted in substantial progress in numerous areas of research (Mattay \& Weinberger, 1999; Rowe \& Frackowiak, 1999), despite the fact that more perceptionoriented studies still dominate the neuroscience literature (Illes et al., 2003). Partially due to limitations imposed by the Magnetic Resonance (MR) scanner environment, research in the motor domain typically employs button-press responses as the behavioral counterpart for the imaging data being obtained. However, this strong emphasis on discrete finger movements does not do justice to the rich repertoire of actions that humans are capable of performing (Sanes, 2003).

Recognizing these limitations, several efforts to record additional data on movement kinematics while scanning have been reported in the literature. Examples of more sophisticated kinematic registration techniques range from angular joint motion recordings (Debaere et al., 2004) or handgrip force measurements (Liu et al., 2000), to the use of video cameras to provide visual feedback (Leube et al., 2003) and input devices like fiber optic joysticks (Miall et al., 2001) and pressure-sensitive air-filled cushions (Jantzen et al., 2004). However, in order to study the learning of complex two-dimensional movement sequences as performed in more natural continuous pen movements, different requirements need to be met which go beyond the capabilities of the aforementioned techniques. Foremost, these requirements pertain to the registration of fast and detailed kinematic measurements of movements along different trajectories. Measurements along predefined 2D-patterns were previously reported by van Mier \& Petersen (2002), using a light-emitting pen and a conventional video camera placed outside the MR scanner room. To allow a more detailed tracking, the new setup introduced here was developed to improve the sampling rate and the spatial resolution in comparison to this earlier attempt, while being compact, having built-in calibration capabilities and being fully MR-compatible.

The need to develop a new setup was evident after confirming that the commercial graphic digitizing tablets we used in previous behavioral experiments fail to work in an MR environment. Although initially a compact and MR-compatible CCD-based optical measurement was considered, the resistance-based recording as presented here was preferred because of its much higher sampling rate at a lower cost. The presented setup enables the recording of continuous pen trajectories related to complex, mechanically predefined, two-dimensional patterns in an fMRI setting. During development, several 
technical challenges needed to be faced, including the handling of unavoidable pressure-related variations in the contact resistance at the tip of the tracing pen. Additionally, the device which enables the tracking of the subject's tracing movements should neither be affected by the scanner's strong static magnetic field, nor by the gradient-switching occurring during Echo Planar Imaging (EPI) sequences. Likewise, the quality of the AMRI data should not be compromised by the operations of the new measurement device. The present paper illustrates the approach that was chosen to overcome these stringent limitations and discusses the validation data showing its feasibility in the context of a maze learning task. In this task, subjects learn to perform new movement sequences by continuously tracing along a path which is defined by a delimiting groove and which constitutes a maze consisting of contiguous straight segments. While keeping their eyes closed during tracing, subjects should solely rely on somatosensory and proprioceptive feedback to learn the novel continuous sequence (van Mier et al., 1998; van Mier et al., 2004). By combining the recording of detailed pen tracing data and the relatively fast image acquisition of AMRI, the relation between behavioral and neural manifestations of learning can be closely monitored over the entire course of skill acquisition.

\section{Materials and Methods}

\subsection{Resistance-based Position Measurement}

The setup's rationale takes advantage of the fact that each trajectory to be traced is basically determined in advance. By predefining the path's general twodimensional geometry, any position along the path can consequently be represented by a one-dimensional variable which indicates the distance covered with respect to its starting point. In our setup, keeping track of the covered distance is achieved by a resistance-based measurement, where the resistance between any current pen position and the path's starting point is proportional to the distance between them.

The mazes which are used in the present application consist of successive straight-line segments which form a closed loop (Figure 1a). The trajectory that should be followed is determined by a groove - the wide dark line in Figure 1a which constitutes the interconnected segments of the maze. Subjects are instructed to learn the novel movement sequence by tracing the path which is dictated by the maze's geometry as fast as possible, solely based on somatosensory information they can extract from the predefined groove and proprioceptive feedback they get while performing these new sequential movements. 
a) maze, maze holder and pen

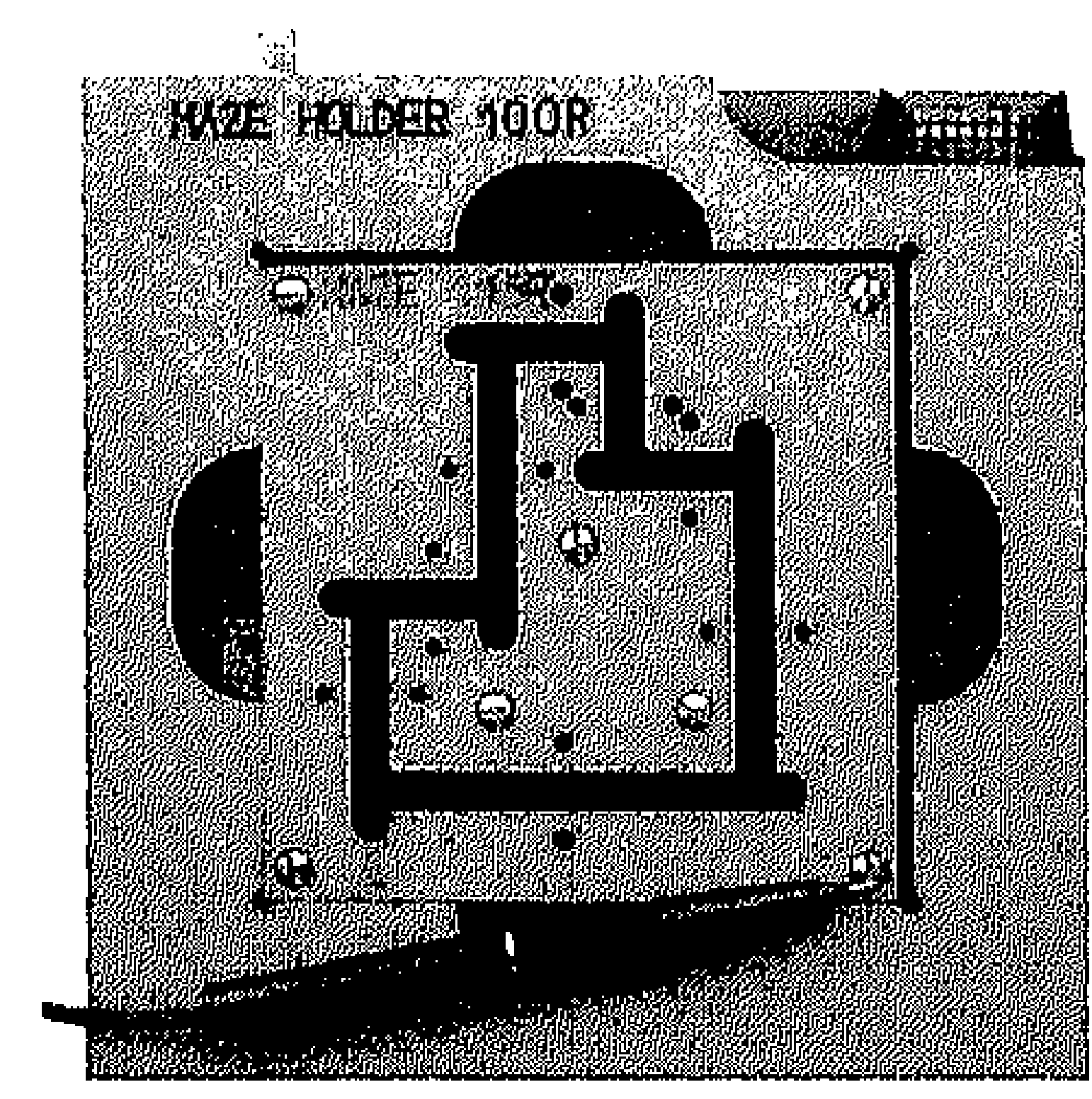

b) schematic setup overview

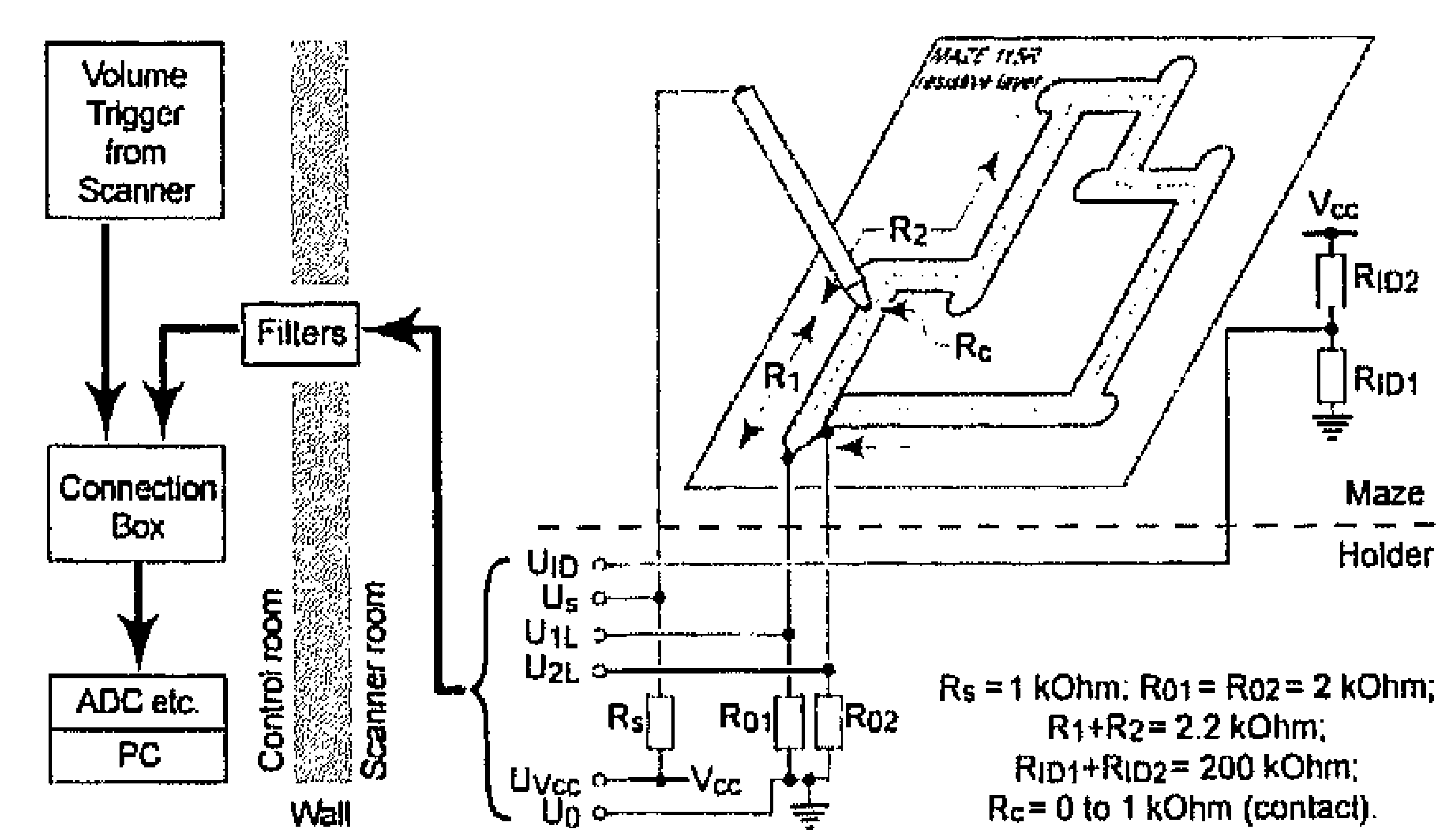

Figure 1. a) A maze which is plugged into the maze holder, together with the adapted pen. Several mazes of $10 \times 10 \mathrm{~cm}^{2}$ are available and readily interchangeable. The actual maze path is a $6 \mathrm{~mm}$ wide guiding groove, which is $3 \mathrm{~mm}$ deep and has a resistive layer at the bottom which is clearly visible here as a dark line. b) A schematic overview of the setup. On the right side of the scanner room wall, the electrical maze circuit is depicted. Note that the maze path acts as a resistor $\left(R_{1}+R_{2}\right)$, and that the variable contact resistance $\left(R_{c}\right)$ is in series to the protective resistor $\mathrm{R}_{\mathrm{s}}$. The components below the horizontal dashed line are located inside the maze holder, including some filtering capacitors not shown here. The connection box and the A/D-converter can be found outside of the scanner room (on the left; see text for details).

Used nomenclature for measured signals: $U_{v c c}$, monitoring of supply voltage $V_{c c} ; U_{I L}$, voltage measured at starting point; $U_{2 L}$, voltage measured at end point; $U_{s}$, voltage measured at pen, as additional constraint; $U_{\mathrm{DD}}$, voltage for online maze identification.

The recording of the subject's pen tracing movements is essentially accomplished in the following way: at the bottom of the groove, a resistive layer forms the surface which the conductive pen tip contacts. The maze path is milled into this resistive layer, a cut is made near the origin to electrically separate the starting- and end-points, and electrodes are connected to both ends (Figure $1 \mathrm{~b}$ ). The total resistance of the formed path has a known and constant value $\left(\mathrm{R}_{\mathrm{t}} \approx 2.2 \mathrm{kOhm}\right)$. As the pen, which simply constitutes a galvanic contact and is connected to a $5 \mathrm{~V}$ power supply via a current-limiting resistor $\left(\mathrm{R}_{\mathrm{s}}\right)$, is moved along the resistive layer, the voltages measured at the electrodes placed at the starting- and end-point of the maze $\left(U_{1 L}\right.$ and $U_{2 L}$ in Figure 1b) decrease/increase accordingly. These voltages allow the reconstruction of the resistances $R_{1}$ and $R_{2}$ between the pen's current position and the start and end of the trajectory, respectively. Noting that:

$$
\mathrm{R}_{\mathrm{t}}=\mathrm{R}_{1}+\mathrm{R}_{2}=\text { constant }
$$

the measured voltages $U_{1 L}$ and $U_{2 L}$ permit to calculate the quantity $R_{1}$ by: 


$$
\mathrm{R}_{1}=\frac{\mathrm{U}_{2 \mathrm{~L}}-\mathrm{U}_{1 \mathrm{~L}}+\mathrm{U}_{2 \mathrm{~L}} \cdot \frac{\mathrm{R}_{\mathrm{t}}}{\mathrm{R}_{02}}}{\frac{\mathrm{U}_{1 \mathrm{~L}}}{\mathrm{R}_{01}}+\frac{\mathrm{U}_{2 \mathrm{~L}}}{\mathrm{R}_{02}}}
$$

where $R_{01}$ and $R_{02}$ are known resistances in series to the movement-dependent resistances $R_{1}$ and $R_{2}$.

Importantly, note that in eq. 2 the contact resistance $R_{c}$ does not appear. This means that by taking into account the relative contribution of both resistances $R_{1}$ and $R_{2}$, adverse effects on the reconstructed $R_{1}$ from unavoidable fluctuations in $R_{c}$ between the pen and the maze's surface (e.g. varying according to the exerted pressure, or to the presence of dirt, or irregularities of the surface) are avoided or at least minimized. Conversely, the values above can also be used to reconstruct $R_{c}$ :

$$
R_{c}=\frac{U_{v c c}-\left(\frac{U_{1 L}}{R_{01}}+\frac{U_{2 L}}{R_{02}}\right) \cdot R_{s}-\left(R_{1}+R_{01}\right) \cdot \frac{U_{1 L}}{R_{01}}}{\frac{U_{1 L}}{R_{01}}+\frac{U_{2 L}}{R_{02}}}
$$

and this actual measurement of the instantaneous contact resistance permits to detect detrimental contact resistance peaks $\left(\mathrm{U}_{\mathrm{vcc}}\right.$ is the monitored supply voltage). Typical values of $R_{c}$ are below $0.1 \mathrm{kOhm}$ (see insert Figure 2), i.e. small in comparison with $R_{s}, R_{01}$, and $R_{02}$ (Figure $1 b$ ). While loosing contact, $R_{c}$ becomes anomalously large. By requiring a low value for $R_{c}$, points included for further analysis are efficiently validated. In this way, the setup fulfills the necessary requirement that every contact loss is correctly recognized as such and hence does not lead to any misinterpretations. Of course, when the pen is lifted from the resistive layer, no position is measurable and the suitability of the validated data available is exclusively determined by the requirements of the data analysis.

Furthermore, the voltage recorded at the point between the pen's protective resistor and the pen's tip, $U_{s}$, provides an additional constraint, as visible from the relation

$$
\frac{U_{v c c}-U_{s}}{R_{s}}=\frac{U_{1 L}}{R_{01}}+\frac{U_{2 L}}{R_{02}}
$$

which yields a continuous monitoring of the measurement accuracy. At a current of about $1.7 \mathrm{~mA}$, the two values measured agree to $(1 \pm 0.22) \%$. 
Based on a calibration procedure carried out previously (see Results), the reconstructed resistances are finally transformed into a measure of the covered distance by:

$$
\mathrm{L}_{1}=\frac{\mathrm{L}_{\mathrm{t}}}{\mathrm{R}_{\mathrm{t}}} \cdot \mathrm{R}_{1}+\mathrm{L}_{0}
$$

where $\mathrm{L}_{0}$ is a constant offset whose effective value is related to the shape of the ends of the resistive strip. An example of the resulting $L_{1}$ curves that can be obtained is shown in Figure 2.

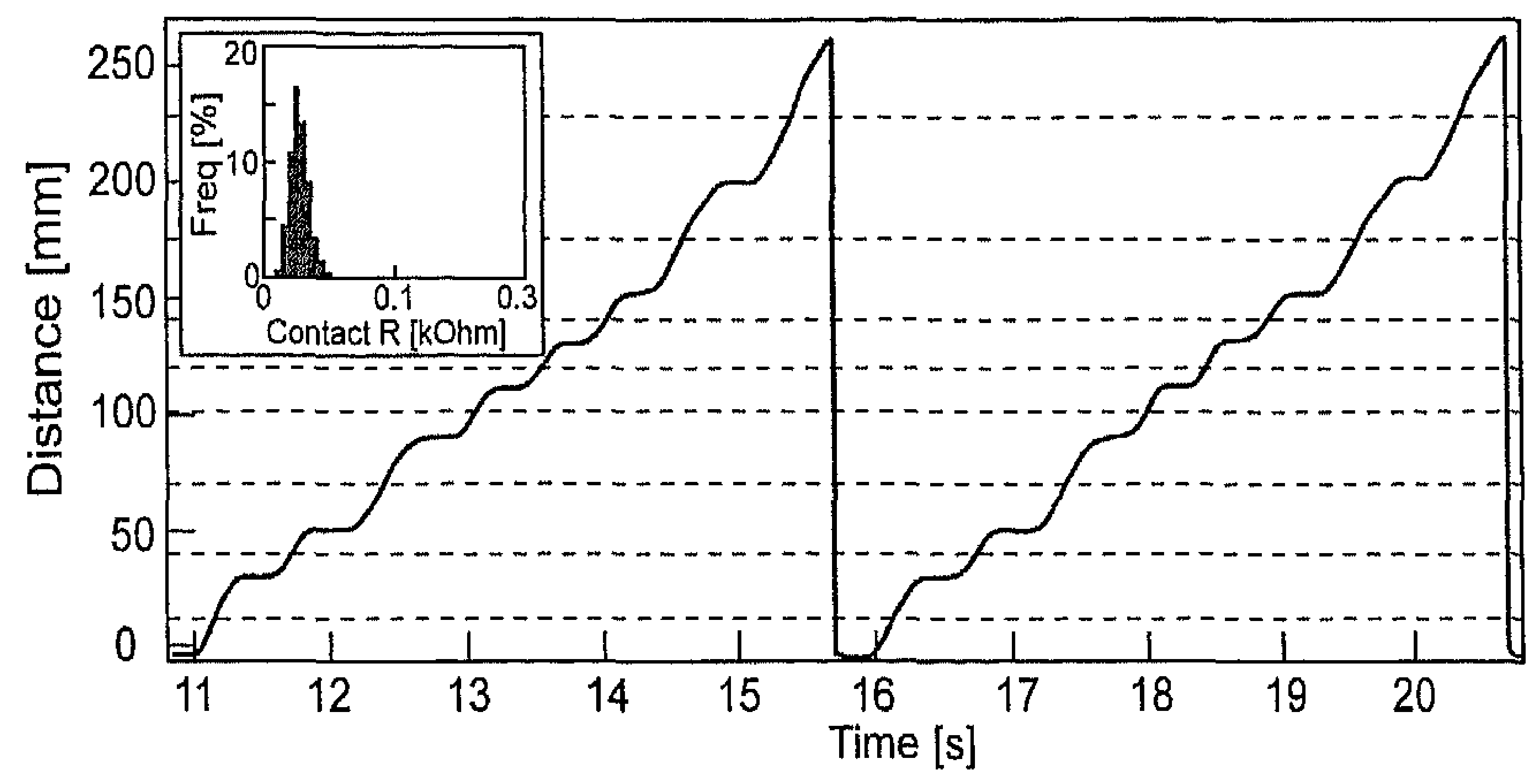

Figure 2. The reconstructed pen position is shown as a function of time for two consecutive loops through the maze (preprocessed data from a run during EPI image acquisition). The dashed lines indicate the location of the eight calibration positions, which also constitute the boundaries of the functional segments during data analysis. Furthermore, note the short plateaus which are related to the slowing down in the corner regions. The insert on the left shows the distribution of the contact resistance values for a run in which six loops were performed.

The basic components of the system needed to realize the resistance-based recording, and further information about the acquisition process itself are discussed in detail in the next sections.

\subsection{Setup Components}

\subsubsection{Mazes}

A series of five mazes was designed. Irrespective of their individual geometries, all mazes are composed of eight straight segments that form a $260 \mathrm{~mm}$ long path. An example is shown in Figure 1. At each intersection, a so-called 'dead- 


\section{Chapter 2}

end' is included to enforce a choice with regard to the direction in which to proceed. Moreover, the end of the maze path coincides with its starting point, constituting a closed loop which can be traced continuously (van Mier et al., 1993).

Each maze consists of four separate layers: The plastic maze layer on top contains a $6 \mathrm{~mm}$ wide, precision-milled groove that defines the trajectory to be traced. Furthermore, a pair of holes is present half way on each segment, to position a small tool permitting calibration of the data by measuring at predefined reference positions. The second layer consists of a $0.12 \mathrm{~mm}$ thin, resistive carbon-loaded plastic foil (Makrofol KL3-1009, Bayer, Leverkusen, Germany), machined along the outline of the maze path. Prior to machining, the resistive foil has been glued onto the third layer, which is a rigid Printed Circuit Board (PCB) that also holds the pins which establish the contacts of both ends of the resistive foil to the maze holder (see next section). Furthermore, the PCB holds two resistors which serve to identify the maze online, as well as their respective contact pins and a contact pin to the shielding ground of the PCB. Finally, a thick plastic base plate at the bottom houses all contact pins and has four 'key' holes which help to unambiguously orient the maze when inserting it into the maze holder, without endangering any of its contacts.

\subsubsection{Maze Holder}

The mazes can be plugged into the maze holder (Figure 1a), where the remaining leads, resistors, capacitors and connectors that complete the setup's intrinsic wiring are located. The maze holder also consists of four assembled layers, of which the PCB layer holds the female contacts for the maze's signal pins, together with further protective resistors and filtering capacitors. Additionally, it includes connectors for the pen and for the readout and voltage supply cable. By design, the maze holder offers the flexibility which is necessary to efficiently switch between mazes within a few seconds.

\subsubsection{Pen}

The tracing is performed with a conductive pen, which is an integral part of the setup's electrical circuit (Figure 1). It was adapted to provide the necessary connection to the maze holder, without sacrificing the natural feel of a pen. The pen's tip is a $2 \mathrm{~mm}$ diameter conductive sphere which can be smoothly moved along the maze path.

\subsubsection{Cabling, $R F$ Filter and Connection Box}

A $9 \mathrm{~m}$ (inside scanner room) plus $3 \mathrm{~m}$ (outside scanner room) long shielded twisted-pair cable is used for readout of the measured signals and to provide 
supply voltage to the maze. The cable shield, including its extension underneath the maze, is connected to the shielding wall of the scanner room to shield the maze signals from the MR-related ones. To further avoid the leakage of highfrequency noise into and out of the scanner room, each line separately passes a custom-built RF low-pass filter which has been mounted on the MR system's Faraday cage (pass band: DC - $30 \mathrm{kHz}$ ).

A purpose-built Connection Box finally serves to route the signals from the maze, the MR scanner and the interface board output to the multi-channel $A / D$ converter section of the interface board (Figure 1b).

\subsubsection{A/D-Converter and Computer}

Data acquisition is performed with a multi-channel, 16-bit A/D-converter sampling at $200 \mathrm{kHz}$ (model NI PCI-6014; National Instruments, Austin, USA). A range of $0-5 \mathrm{~V}$ is covered across all channels, resulting in a resolution of 0.1 $\mathrm{mV}$. The A/D-converter interface board is installed in a PC (Optiplex GX260, Dell), with $512 \mathrm{MB}$ RAM and a $2.4 \mathrm{GHz}$ processor, running data acquisition and display software developed with LabVIEW v7.0 (National Instruments, Austin, USA) under Windows XP.

\subsection{Data Acquisition and Processing}

Simultaneous to the measurement of $U_{1 L}$ and $U_{2 L}$ (at the respective ends of the maze path) and $U_{s}$ (at the point between the pen's protective resistor and the pen's tip), the $5 \mathrm{~V}$ input to the pen $\left(\mathrm{U}_{\mathrm{vcc}}\right)$ and the voltage of the identification resistors of the currently traced maze $\left(\mathrm{U}_{\mathrm{ID}}\right)$ are monitored.

For offline synchronization between tracing data and MR images, the scanner's pulses which mark the start of each multi-slice MR volume acquisition are fed into the sixth analog channel of the $A / D$ converter. Since phases of tracing activity and rest are alternated during the fMRI measurements, a high or a low tone which is issued via the computer's audio output tells the subject when to start or stop tracing, respectively. In order to time-lock the alternation between these phases to the MR volume acquisition, the scanner's volume triggers are fed into a counter on the interface board which switches its output state after a predefined number of pulses. This output state transition is used to generate the instructional tone for the subject, and the counter's output level is passively added to the input of the sixth analog $A / D$ channel mentioned earlier for offline synchronization.

For all six analog channels mentioned above, acquisition takes place in differential mode, implementing an averaging procedure across 10 successive samples (out of sets of fifty) per logged data point. The 10 samples used for 1 data point correspond to a measuring time of $0.3 \mathrm{~ms}$ and are acquired every 1.5 
ms. Typically, the obtained standard deviation across the averaged 10 samples is well under $1 \mathrm{mV}$, although in a small fraction of disturbed data points much larger values can be observed. Together with the averages, the standard deviations are also logged, as they are indicative of the data quality at this local level. A cross-check at the filtering phase has confirmed that data points discarded during this stage also initially had a large standard deviation in the raw samples. At an effective event logging rate of $\sim 666 \mathrm{~Hz}$ per channel, the raw data are written to an ASCII file for subsequent processing.

Data preprocessing involves the following steps: exclusion of samples with too large $(\geq 4 \mathrm{kOhm})$ contact resistance values (constituting a small fraction of the entire distribution, see insert Figure 2), exclusion of samples where $U_{1 L}+U_{2 L}<$ $2 \mathrm{~V}$ indicating (partial) contact losses, noise peak detection by determination of signal smoothness, and FFT-based low-pass filtering. Whenever a sample is discarded during preprocessing, linear interpolation is used to replace the affected data point, based on the fact that the high sampling rate ensures that neighboring samples are clustered closely enough in space to render linear interpolation a valid method. Furthermore, the reconstruction of the pen's position $\left(L_{1}\right)$ across time is obtained from the $R_{1}$ values. The value of $R_{1}$ (eq. 2) is reconstructed from the values of $U_{1 L}$ and $U_{2 L}$, measured at each time point, and the constant values $\mathrm{R}_{01}, \mathrm{R}_{02}$, and $\mathrm{R}_{\mathrm{t}}$. Similarly, the setup's redundant measurement feature permits to obtain two further nearly independent reconstructions of $R_{1}$, based on the values of $U_{s}$ and $U_{v c c}$, together with either $\mathrm{U}_{1 \mathrm{~L}}$ or $\mathrm{U}_{2 \mathrm{~L}}$. The values of the position obtained by these additional reconstructions agree with the value from eq. 2 to better than $0.31 \pm 0.16 \mathrm{~mm}$ at every point, which is indicative for the overall measurement accuracy. At points disturbed by a partial contact loss, the difference can increase to up to $1.5 \mathrm{~mm}$ because the two further reconstructions are more affected than the differential method based on eq. 2, which is used per default. To complete the preprocessing procedure, these reconstructions are thus compared with the main reconstruction to determine the overall data consistency and measurement accuracy; an average difference below $0.6 \mathrm{~mm}$ is taken as a criterion for goodness.

During subsequent data processing, relevant behavioral parameters are extracted separately for each traced segment. The main performance variables extracted from the measured local velocities are the mean velocity achieved per segment, the number of identified stops, and the level of isochrony across different segment lengths. One option for the segment-wise report of these variables is based on a segmentation scheme in which the border of a functional segment is located in the middle of the maze's straight-line segments. This location is advantageous compared to the more natural straight-line segments' ends because the exact time of passage through a corner boundary is less well defined, both due to the subject's possible errant movements in this region and 
to a larger uncertainty in the position reconstruction. Conversely, the extraction of relevant parameters can also be performed for the straight-line segments by defining their boundaries to lie near the known corner regions, and hence distinguishing the achieved velocity along a straight segment from the decision time at each corner. A further calibration tool (similar to the one shown in Figure 3) serves to obtain an accurate measurement of the necessary reference points at these boundary locations. In addition to the change in direction and the dead-end at each corner - which already impede simply sliding along the borders without learning the maze shape -, the use of the borders as a fast guidance along the straight lines can further be penalized by having waveshaped borders. To perform both the preprocessing and the extraction of relevant behavioral parameters on a segment-by-segment basis, we developed a program and a Graphical User Interface with Matlab (v6.5 R13; The Mathworks, Natick, USA).

\subsection{Validation Process}

The validation of the described setup took place in three successive steps. Initially, the question whether the reconstructed resistances could accurately reflect the covered distance along the maze path was addressed in a series of calibration measurements. Subsequently, the impact of the potential mutual interference between the maze setup and the MR scanner needed to be assessed. Therefore, the calibrations were repeated in an MR environment, together with the recording of actual loop tracing data. Data collected outside of the scanner room were used as a baseline reference to which the other measurements could be compared. Finally, the quality of the functional MR images needed to be ensured by showing that the maze setup would not interfere with the scanner's functioning. On-site tests of the setup in an MR environment were mainly performed on a 1.5 Tesla whole-body system and later extended to a 3T scanner setting (Magnetom Sonata and Trio, Siemens Medical Systems, Erlangen, Germany). Whenever functional images were acquired, a standard EPI sequence was used (typical settings: $\mathrm{TR}=2000 \mathrm{~ms} ; \mathrm{TE}=40 \mathrm{~ms} ; 90^{\circ}$ flip angle; 25 slices with $3.5 \times 3.5 \times 4.5 \mathrm{~mm}^{3}$ voxel size and a $224 \times 224 \mathrm{~mm}^{2}$ Field of View (FoV)).

\section{Results}

\subsection{Calibration Measurements}

As first calibration runs outside of the scanner, data were recorded from eight predefined positions along the maze path (Figure 3a), making use of a tool 


\section{Chapter 2}

which was designed to accurately trace these positions perpendicularly to the general tracing direction (Figure $3 \mathrm{~b}$ ). On the one hand, the resulting data are a direct measure of the spatial resolution achieved. On the other hand, the linear relation between the measured resistance and the covered distance can be shown and quantified.

While holding the pen at a given fixed position without moving it, the observed fluctuations for the measured resistance were within a range of $\sim 0.0005 \mathrm{kOhm}$ (i.e. about $0.02 \%$ of the total maze path resistance of $2.2 \mathrm{kOhm}$ ). When tracing movements from the inner to the outer maze border and vice versa were performed at a given distance from the origin, the variations increased to a range of up to $\sim 0.0015 \mathrm{kOhm}$, i.e. $\sim 0.07 \%$ of the total resistance and about 0.2 $\mathrm{mm}$ in reconstructed position, reflecting the uncertainty due to the maze path's width (insert Figure 3a). These geometrically induced variations are treated as fluctuations in the real tracing measurements, given that no information is available on where the pen was actually passing with respect to the maze path's borders. The range of $0.0015 \mathrm{kOhm}$ is then treated as an error of \pm 0.00075 $\mathrm{kOhm}$, i.e. as a standard deviation of $0.1 \mathrm{~mm}$. Despite these increased fluctuations related to the maze geometry, the observed findings still clearly point to a sub-millimeter resolution for the ultimately calculated distances.

a)

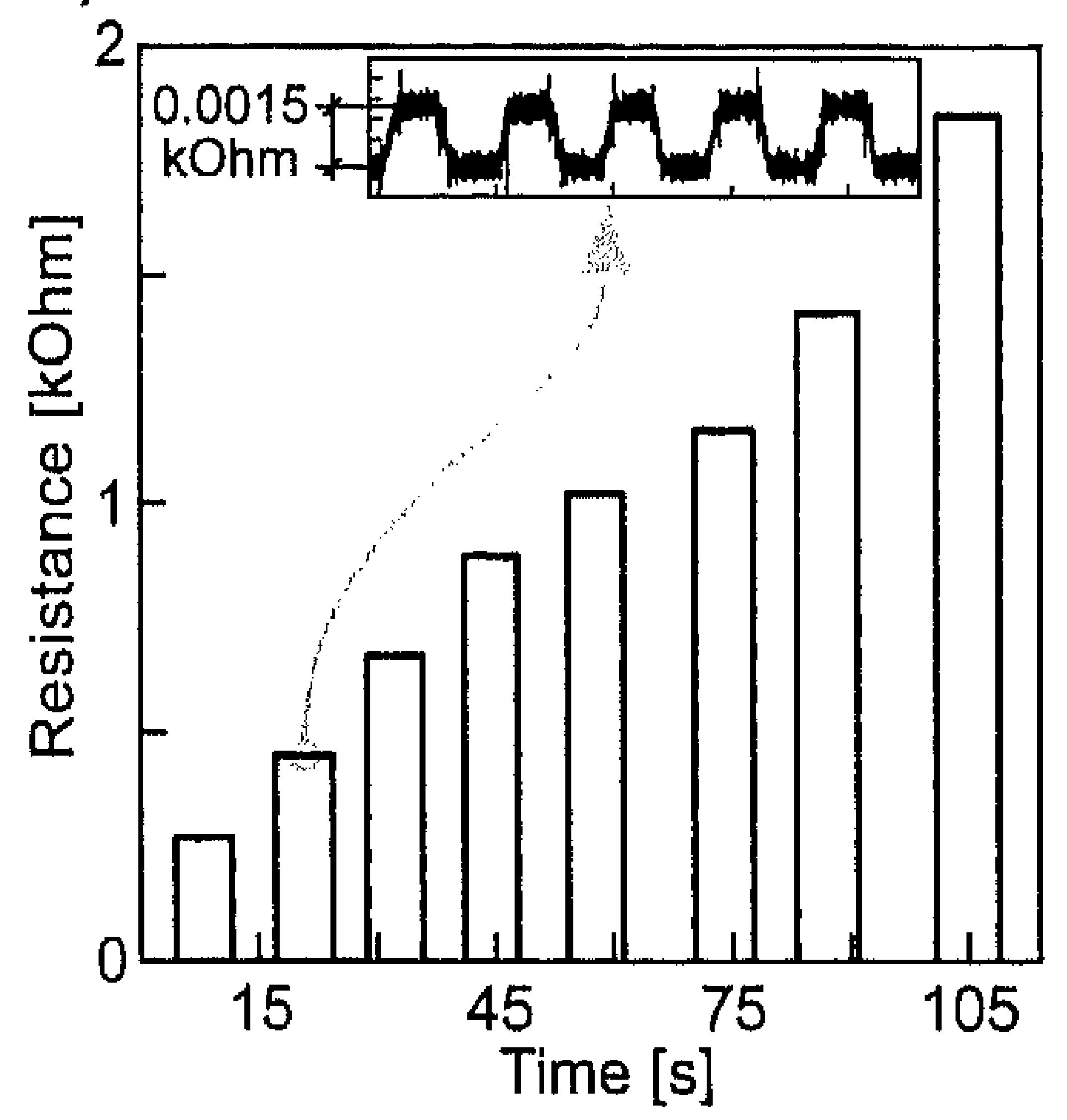

b)

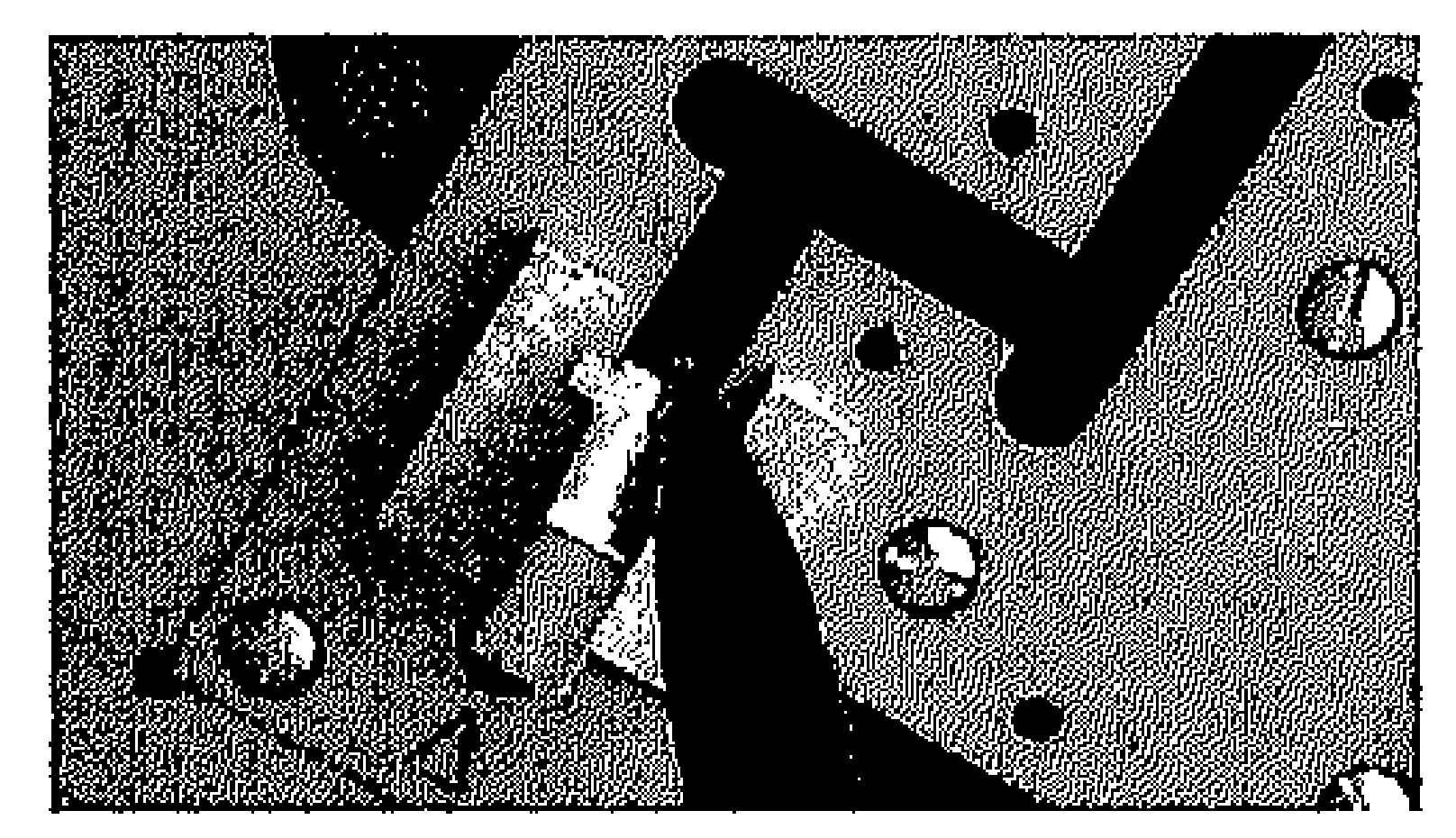

c)

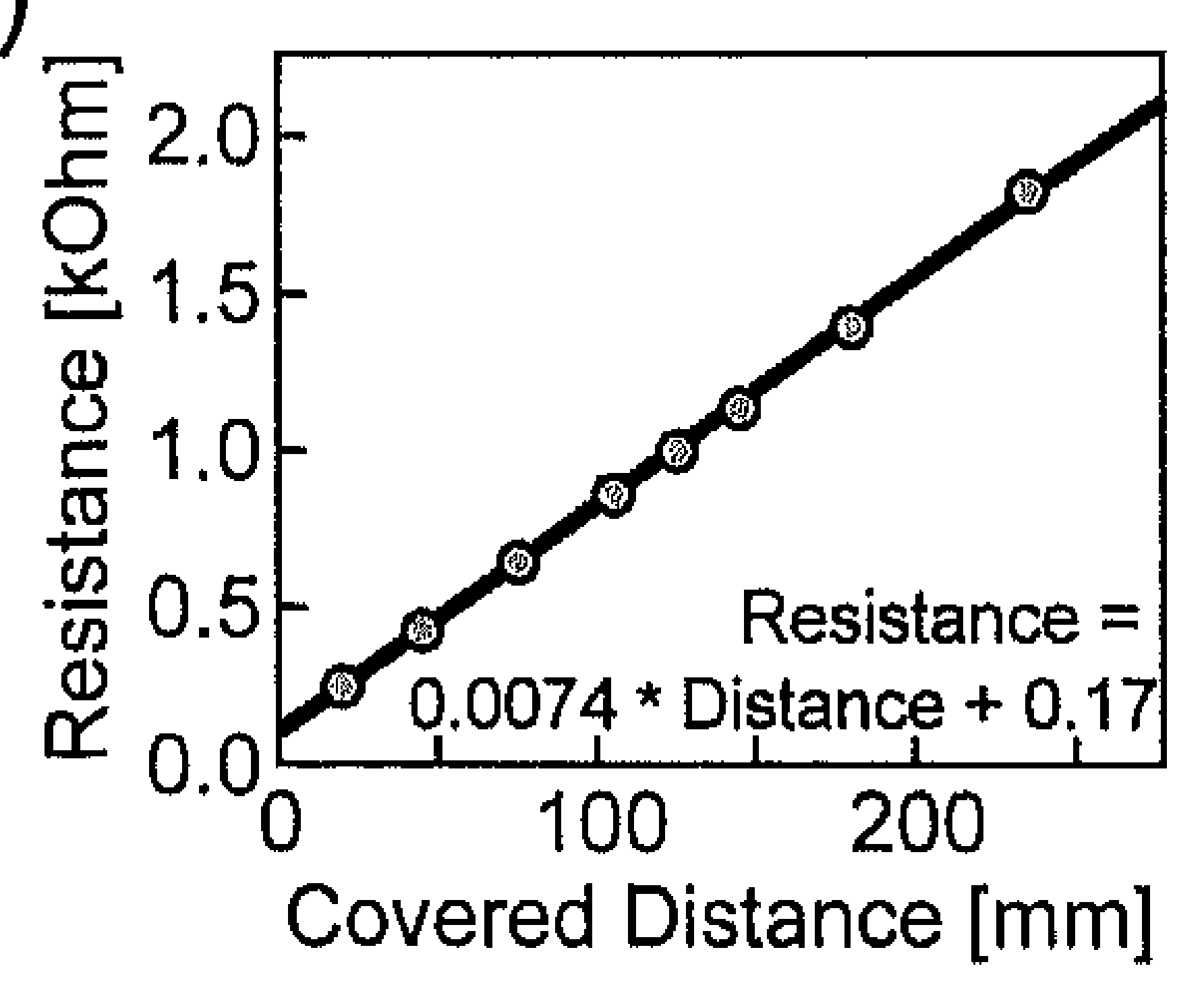

Figure 3. Calibration data. a) Resistances for eight predefined positions recorded outside of the MR scanner room. The insert indicates that the variations observed are mostly due to the maze path's width (showing five repetitions of going back and forth between the maze's inner and outer border at the second calibration position). Note that the reconstructed value of the maze resistance can fluctuate largely and even drop below zero whenever the pen is lifted; such points are characterized by large $R_{c}$ and low $U_{1 L}+U_{2 L}$ values and are safely removed by the filtering procedure. b) A photograph illustrating the use of the calibration tool. The small holes visible half 
way on each segment ensure precise positioning of the calibration tool, which serves to move the pen across the path's width at predefined positions. c) The linear relation between covered distance and measured resistance for the eight calibration positions, as obtained from measurements during EPI image acquisition.

The reproducibility was obtained by repeating the calibration measurements, where the measured resistances were found to agree within $0.0005 \mathrm{kOhm}$ on average. The absolute precision at the calibration points is obtained by folding the reproducibility with the error from fluctuations, leading to $0.0009 \mathrm{kOhm}$ or $0.11 \mathrm{~mm}$.

Another important result is that the calibration data show a strong linear interrelation between the measured resistance and the covered distance with respect to the maze's starting point. Imposing an exact linear relation between the two quantities leads to residuals of about $1.2 \mathrm{~mm}$ rms. In other words, the pen's location can be reliably extracted from the measured signals along the entire path. Besides, the calibration procedure would assure the correctness of the performed measurements even in the light of slight local deviations from this generally applying strict linear interrelation, because the reference points for calibration are located at the points which are mainly used for extraction of the performance parameters during later runs.

\subsection{Effect of MR Environment on Tracing Data}

To verify the setup's proper functioning in an MR environment, the calibration runs were repeated inside the scanner room. The measurements were performed at the scanner-bore's opening, about an arm-length away from the magnet's isocenter. When compared to the measurements outside of the scanner room, the observed fluctuations for the eight predefined calibration positions along the maze were unchanged in the static magnetic field of the scanner, but were $\sim 1.5$ times larger whenever EPI images were acquired. The precision at the calibration points is therefore worsened to about $0.2 \mathrm{~mm}$. Nevertheless, in all cases, the pen tip location could be determined with at least millimeter resolution (corresponding to about $0.008 \mathrm{kOhm}$ ) after preprocessing. In terms of spatial resolution, the setup therefore clearly meets the necessary requirements for the extraction of detailed behavioral data. In addition, the earlier identified strong interrelation between reconstructed resistance on the one hand, and predefined distance on the other, remained unchanged in the MR scanner's proximity (Figure 3c). Similar to the results obtained outside of the scanner room, residuals of about $1.2 \mathrm{~mm} \mathrm{rms}$ were observed in both the $1.5 \mathrm{~T}$ and $3 \mathrm{~T}$ scanner settings.

When considering actual tracing data, where continuous loops are repeatedly traced through a given maze, the first observation is that one can 
unambiguously identify the resulting pattern as the traced trajectory (as already illustrated in Figure 2). Yet, two recurring types of perturbation are recognizable in the raw data. First, the constantly superimposed noise that is related to measurements during EPI image acquisition is moderate, but clearly visible (Figure 4a). A far more drastic source of noise are sporadically occurring partial or full contact losses between the pen and the traced surface, which can arise in either setting (i.e., both inside and outside of the scanner room). Either caused by a confined roughness of the maze's resistive layer or merely by the subject's movements, these contact losses can lead to considerable noise peaks. However, due to the redundant measurement permitting to cope with a varying contact resistance, and the high data acquisition rate at which the maze signals are recorded, the preprocessing procedure can successfully remove these undesirable fluctuations and still yield a detailed tracking even in extreme cases (Figure $4 \mathrm{~b}$ ). In general, no more than $10 \%$ of the samples acquired during tracing are discarded.
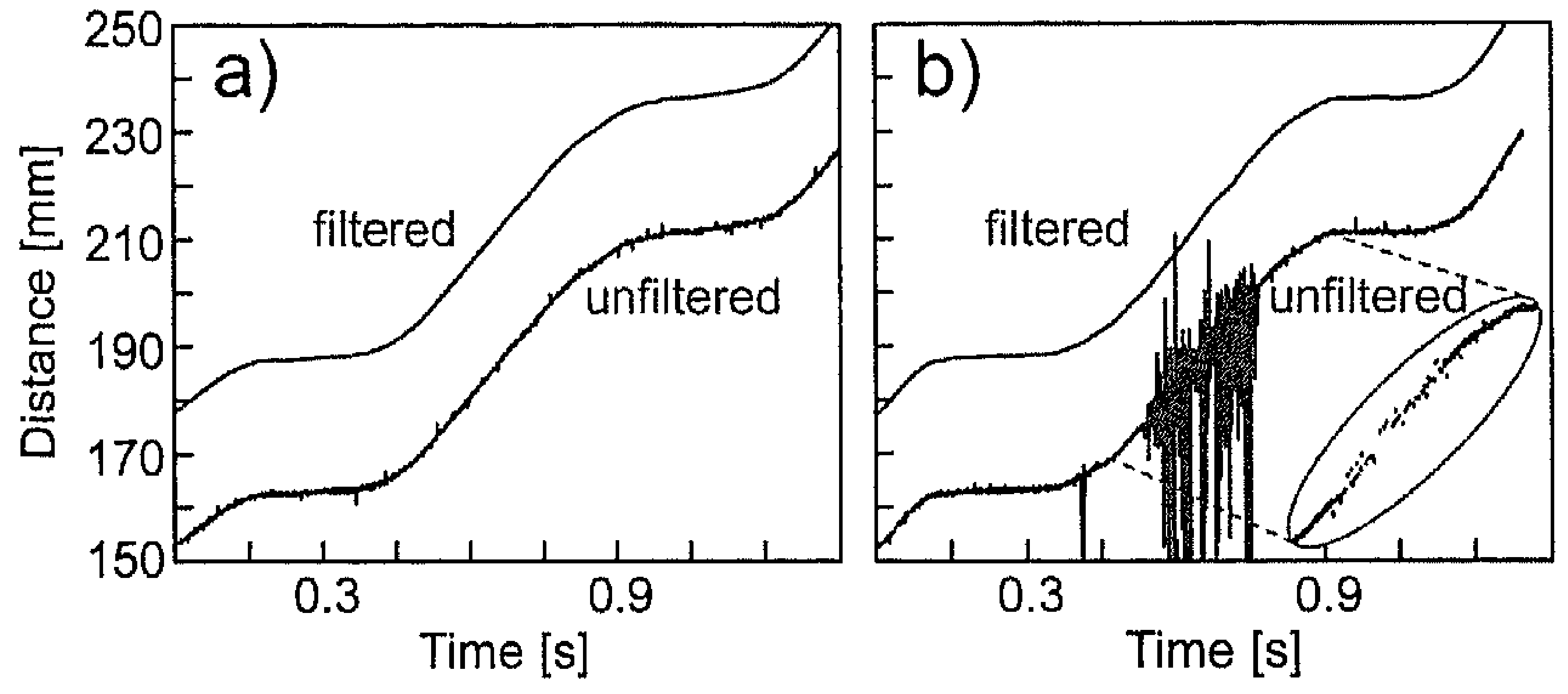

Figure 4. Enlarged part of a measurement, illustrating generally observed noise levels. a) The typical noise visible on raw data (red curve) is modest and is filtered out effectively (green curve, displaced vertically). b) Even extreme disturbances of raw data points (red curve), related to loosing contact, can be filtered out without significant distortion (green curve, displaced vertically). The insert on the right shows the raw values of the points passing the filtering procedure. Due to the redundant measurement and the high sampling rate, sufficient undisturbed data points $(\sim 33 \%)$ are still available for reconstruction in this affected region.

\subsection{Effects of Tracing Setup on MR Image Quality}

The results discussed so far have dealt with the validation of the method per se, and the proper functioning of the resistive setup in the MR scanner's proximity. Conversely, there is also a need to establish that the introduced setup does not 
adversely impact the scanner's functioning. To assess potential effects on functional image quality, several test measurements were performed.

Using a phantom, the Signal-to-Noise Ratio (SNR) was dynamically determined according to the procedure described by Weisskoff (1996). The first result concerned the 'best' SNR, which is defined as the ratio between the average of the mean signal intensity within a relatively large Region of Interest (ROI) in the middle of the image on the one hand, and the corrected average of the standard deviation of the intensity within a background noise ROI on the other hand, across all acquired functional volumes. With respect to this 'best' SNR, the measurements in the baseline condition $\left(\mathrm{SNR}_{0}=146\right)$ and the condition in which resistive measurements were performed with and without tracing $\left(\mathrm{SNR}_{0}\right.$ $=148$ ) did not differ significantly in the light of generally observed $\mathrm{SNR}_{0}$ fluctuations (a mean $\mathrm{SNR}_{0}$ of 144 (std $=2.9$ ) was found across 35 quality assurance measurements ranging from four months prior to three months after the measurement discussed here). Furthermore, calculating the relative fluctuations across scans for several ROIs with increasing linear size (Figure 5) for both conditions also indicated that the presence and use of the maze setup components did not affect the scanner's stability with regard to functional measurements. The fluctuations in the baseline run amounted to $0.61,0.12$ and 0.09 percent of the mean signal intensity for the $1 \times 1,7 \times 7$ and $15 \times 15$ pixel ROIs respectively (Figure 5a). While the maze setup was present and in use, the observed fluctuations added up to $0.59,0.15$ and 0.11 percent for the same ROIs (Figure 5b).

Additionally, a 'spike check' procedure which is part of the scanner's quality assurance protocols was executed. No spikes which might point to additional $\mathrm{RF}$-interference patterns related to the operation of the tracing setup could be identified. 
Chapter 2

a)
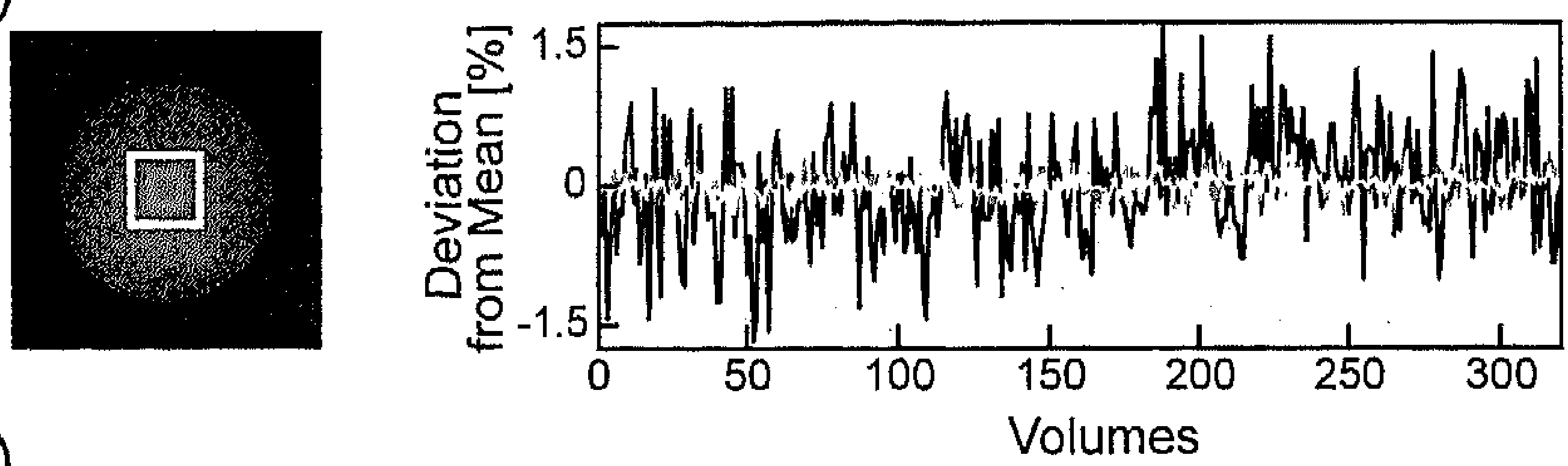

b)
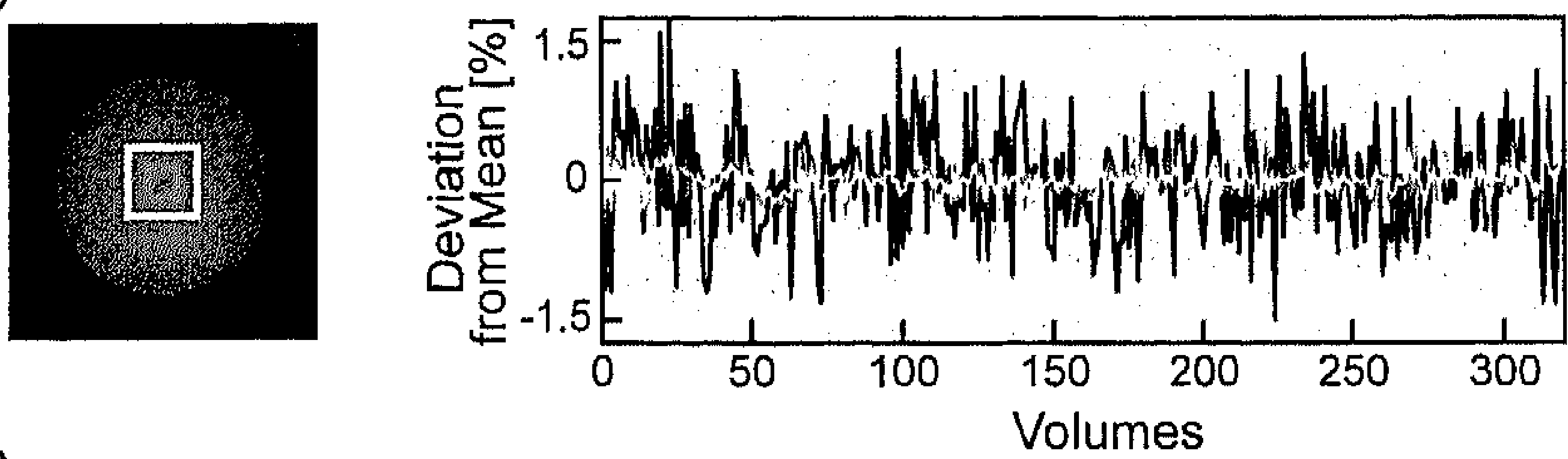

c)
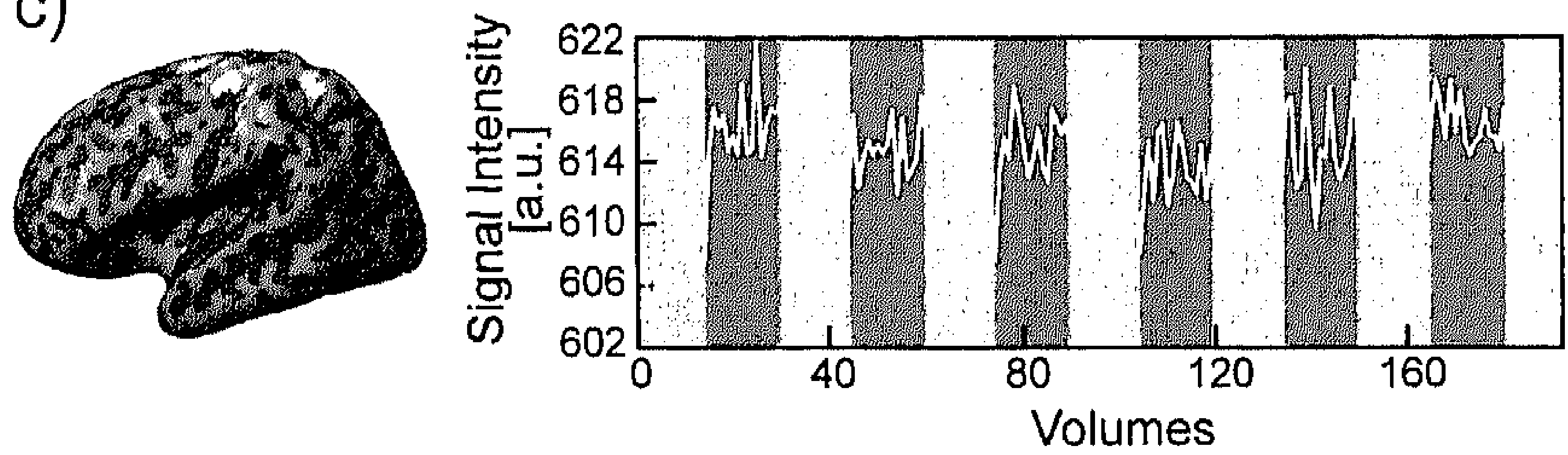

Figure 5. Phantom slice from the stability measurements a) without and b) with the maze setup present and in use. The corresponding plots on the right show the deviation from the mean signal intensity for 3 different ROIs: a $1 \times 1$, a $7 \times 7$ and a $15 \times 15$ pixel ROI (depicted in dark to light blue respectively), indicating that the deviations are similar in range in the two conditions, and that fluctuations decrease with increasing ROI size as expected.

The lower panel c) shows the left hemispheric activation during tracing, together with the extracted time course for M1 (light grey indicates rest condition). Note that no adverse effects due to the tracing setup's operation are observable in the fMRI time series.

Finally, functional images were acquired during an actual experimental run involving a single subject. The run consisted of two alternating conditions, presented in a blocked fashion: an active tracing condition and a baseline rest condition, each lasting $30 \mathrm{~s}$. A switch between conditions was indicated by one of two tones generated with the LabVIEW software. In total, 6 repetitions of active tracing were performed. The maze setup was installed as described in the previous sections. The maze holder was fixed on a custom-built MR-compatible table. Before starting the experiment, the position of the table was adjusted so that the subject could perform the required movements rather effortlessly. Tracing was performed with the dominant right hand. The obtained imaging data were preprocessed and analyzed using BrainVoyager QX (Brain 
Innovation, Maastricht, The Netherlands). The resulting motion correction parameters indicated that head motion was restricted to less than $1.5 \mathrm{~mm} / 1.5$ degrees in any of the possible translation/rotation directions. The deviations induced by phasic task-correlated motion were minimal $(<0.5 \mathrm{~mm} / 0.5$ degrees $)$ and do not confound any learning-related changes in brain activity which might be observed over time. When contrasting the active tracing with the baseline rest condition, a network of activated regions including the primary motor cortex (M1), Supplementary Motor Area (SMA), Pre-Motor Area (PMA), posterior parietal areas and the cerebellum was identified (Figure $5 \mathrm{c}$ ), in accordance with general expectations based on earlier work (van Mier et al., 1998). Close inspection of the time courses from selected ROIs furthermore indicated that the simultaneous recording of the subject's tracing movements did not have any noticeable effect on the quality of the functional images. This observation was further substantiated by successful application of the resistive tracing setup in the 3T MR scanner environment.

\section{Discussion}

Generally, the interpretation of neuroimaging data heavily relies on concurrently recorded behavioral measurements. Unfortunately, the MR scanner environment poses severe constraints on the approaches that can be used to collect behavioral data while scanning. Whereas simple discrete button-press responses are recorded in the majority of studies on motor control and learning, several complementary kinematic registration techniques have been recently reported in the literature. In order to further enlarge the diversity of available kinematic measurements, the resistive setup presented here was developed to enable the recording of continuous pen tracing data along predefined trajectories in an fMRI setting. In the present setup, reconstruction of the pen's position across time is performed with high spatial resolution of $0.2 \mathrm{~mm}$ and temporal resolution of $0.3 \mathrm{~ms}$, is independent from the varying contact resistance between the pen and the resistive surface, and is further ensured by a redundant measurement scheme and built-in calibration features. To the best of our knowledge, no alternative setup suitable for such fast and precise measurements has been reported. Furthermore, the setup can be designated 'MR compatible' in the sense that it is MR safe, it operates as designed in the MR environment, and finally does not have any negative effects on the image quality of the acquired fMRI scans. Although the present work focuses on the applicability to the measurement of more complex two-dimensional movement sequences along predefined patterns, the resistive method's general principles can also be applied to record simpler movements, e.g. when the rotation around a given axis 


\section{Chapter 2}

should be monitored (as during joint flexion/extension or during evaluation of haptic matching performance where two objects need to be aligned within a specified plane).

Beyond the currently intended application, the introduced resistive method furthermore has the potential to be adapted to support general unrestricted pen tracing in two dimensions by measuring the voltages at the four corners of a square resistive layer. In case of more modest requirements on resolution and sampling rate, and depending on space available, a CCD-based readout of traces performed with a light-carrying pen might alternatively be considered.

In summary, the presented setup helps to thoroughly investigate the relation between behavioral and neural manifestations of learning a complex novel movement sequence throughout the entire course of learning and in the context of several paradigmatic variations (e.g., intermanual transfer, passive learning etc.). By allowing the detailed and temporally highly resolved recording of tracing movements during fMRI data acquisition, the introduced method extends the range of phenomena which can be studied in the domains of motor control and learning.

\section{Acknowledgments}

The authors are grateful to J. Grooten, G. Hilgers, R. Lampe and W. Reuter of the RWTH Aachen University for their help during the design and manufacture of the mazes and the maze holder. We also thank P. Gaalman and E. van den Bergh for their assistance in the validation of the presented setup in the MR environment. Furthermore, we acknowledge assistance from H. Hamers and the faculty of Psychology's technical staff at Maastricht University. 


\section{References}

Debaere, F., Wenderoth, N., Sunaert, S., Van Hecke, P., \& Swinnen, S.P. (2004). Cerebellar and premotor function in bimanual coordination: parametric neural responses to spatiotemporal complexity and cycling frequency. NeuroImage, 21, 1416-1427.

Illes, J., Kirschen, M.P., \& Gabrieli, J.D.E. (2003). From neuroimaging to neuroethics. Nature Neuroscience, 6, 205.

Jantzen, K.J., Steinberg, F.L., \& Kelso, J.A.S. (2004). Brain networks underlying human timing behavior are influenced by prior context. Proceedings of the National Academy of Sciences USA, 101, 6815-6820.

Leube, D.T., Knoblich, G., Erb, M., Grodd, W., Bartels, M., \& Kircher, T.T.J. (2003). The neural correlates of perceiving one's own movements. NeuroImage, 20, 2084-2090.

Liu, J.Z., Dai, T.H., Elster, T.H., Sahgal, V., Brown, R.W., \& Yue, G.H. (2000). Simultaneous measurement of human joint force, surface electromyograms, and functional MRI-measured brain activation. Journal of Neuroscience Methods, 101, 4957.

Mattay, V.S., \& Weinberger, D.R. (1999). Organization of the human motor system as studied by functional magnetic resonance imaging. European Journal of Radiology, 30, 105-114.

Menon, R.S. (2001). Imaging function in the working brain with fMRI. Current Opinion in Neurobiology, 11, 630-636.

Miall, R.C., Reckess, G.Z., \& Imamizu, H. (2001). The cerebellum coordinates eye and hand tracking movements. Nature Neuroscience, 4, 638-644.

Rowe, J.B., \& Frackowiak, R.S.J. (1999). The impact of brain imaging technology on our understanding of motor function and dysfunction. Current Opinion in Neurobiology, 9, 728-734.

Sanes, J.N. (2003). Neocortical mechanisms in motor learning. Current Opinion in Neurobiology, 13, 225-231.

Savoy, R.L. (2001). History and future directions of human brain mapping and functional neuroimaging. Acta Psychologica, 107, 9-42.

van Mier, H., Hulstijn, W., \& Petersen, S.E. (1993). Changes in motor planning during the acquisition of movement patterns in a continuous task. Acta Psychologica, 82, 291312. 


\section{Chapter 2}

van Mier, H., Tempel, L.W., Perlmutter, J.S., Raichle, M.E., \& Petersen, S.E. (1998). Changes in brain activity during motor learning measured with PET: Effects of hand of performance and practice. Journal of Neurophysiology, 80, 2177-2199.

van Mier, H., \& Petersen, S.E. (2002). Role of the cerebellum in motor cognition. Annals of the New York Academy of Sciences, 978, 334-353.

van Mier, H., Perlmutter, J.S., \& Petersen, S.E. (2004). Functional changes in brain activity during acquisition and practice of movement sequences. Motor Control, 8, 500520.

Weisskoff, R.M. (1996). Simple measurement of scanner stability for functional NMR imaging of activation in the brain. Magnetic Resonance in Medicine, 36, 643-645. 


\section{Chapter 3}

\section{Dynamic neural representations of continuous motor sequences: Effects of intra- and inter-session learning}

Based on: Reithler, J., van Mier, H., \& Goebel, R. (submitted). Dynamic neural representations of continuous motor sequences: Effects of intra- and intersession learning. 
Chapter 3

\begin{abstract}
The acquisition and generation of action sequences constitute essential elements of purposeful human behavior, and are of utmost importance for the successful completion of a multitude of our daily activities. The current functional magnetic resonance imaging (FMRI) study focused on changes in the neural representation of continuous movement sequences as learning evolved. Behavioral and neural manifestations of nonvisual motor practice were studied both within the time frame of a single scanning session, as well as across several days of extended practice. Based on detailed behavioral recordings to discriminate between early and late stages of learning at the single subject level, and by applying advanced inter-subject alignment procedures, sequence-specific decreases in activation throughout a learning-related cortical network of areas were observed already after few minutes of practice. Furthermore, the basic architecture of this network remained unchanged after extensive practice, although further decreases in activation levels could be observed as learning progressed. Only the posterior part of the left putamen showed increased activation levels when an extensively trained sequence needed to be recalled. Overall, these findings imply that learning the required continuous movement patterns is not mediated by widespread reorganizations at the neural systems level, but is mainly associated with more efficient processing in a network of consistently recruited areas.
\end{abstract}




\section{Introduction}

The ability to generate intentional action sequences enables us to freely interact with the world that surrounds us and is therefore crucial with respect to many aspects of our daily lives (Tanji, 2001). Neuroimaging techniques have been successfully applied to localize brain regions involved in the production of willed actions (Culham and Valyear, 2006), and efforts have additionally been directed towards exploring the dynamics within the identified neural networks associated with learning (Poldrack, 2000). However, the reported results concerning learning-related changes have been largely inconsistent. The observed discrepancies are most likely due to a number of factors related to the amount of practice undertaken, the type of task (e.g., sequence learning vs. motor adaptation) and learning (e.g., implicit vs. explicit) involved, the chosen baseline conditions, and the time window in which imaging data have been acquired (Doyon and Ungerleider, 2002; Toni et al., 1998; Ashe et al., 2006). Furthermore, sequence learning has generally been studied in the context of Serial Reaction Time (SRT)-like paradigms (Nissen and Bullemer, 1987), in which each response is specifically coupled to a presented sensory event. This inherent 'associative' component potentially blurs what is actually being learned (Keele et al., 2003; but see Bischoff-Grethe et al., 2004). Finally, there is a strong bias in the motor sequence literature towards the use of discrete button-press tasks (Sanes, 2003; Clegg et al., 1998), which raises the question whether the observed effects also generalize to situations in which a different type of movement sequencing is required.

The present fMRI study focused on continuous movement patterns resembling the smooth succession of coherent motion elements in handwriting, and was concerned with learning at two different time scales: in the order of minutes and across several days. Subjects were instructed to learn a set of 2-dimensional continuous movement sequences by tracing along predefined maze trajectories, while keeping their eyes closed throughout the entire experiment (van Mier et al., 1993). Since no visual information on the desired trajectory was provided, subjects had to solely rely on somatosensory and proprioceptive feedback while performing the required movements. In this way, effects of visual guidance and cognitive learning strategies related to associations with discrete sensory events could be excluded, while concentrating on the motor aspects of learning the novel continuous movement sequences. Furthermore, the evolution of the behavioral and neural manifestations of learning was examined both at the within- as well as the across-sessions level, by including two separate scanning sessions and thereby providing a more complete picture of the studied learning process. The main goal was to investigate whether the chosen time windows would display different kinds of functional reorganization due to learning (see Kelly and Garavan, 2005), by contrasting individually defined early and late phases of learning based on detailed behavioral recordings. 
Chapter 3

The current findings will be discussed in the light of recent models describing the dynamic cerebral changes that are thought to occur during the different stages of skill acquisition (Doyon and Ungerleider, 2002; Doyon and Benali, 2005; Ashe et al., 2006).

\section{Materials and Methods}

\subsection{Participants}

Twelve healthy subjects $(6$ males; mean age $=25$ years, standard deviation $=$ 1.4 years) participated in the current study. All subjects were right-handed as determined by the Edinburgh Handedness Inventory (Oldfield, 1971; mean score $=94$, standard deviation $=9$ ) and gave written informed consent according to a protocol approved by the local research ethics committee.

\subsection{Experimental Paradigm}

\subsubsection{Motor Learning Task}

Subjects were asked to learn a set of new movement sequences by continuously tracing along different predefined maze trajectories. Although each maze had a unique geometry, all mazes shared certain characteristics: the groove to which the pen movements were restricted was $6 \mathrm{~mm}$ wide and $3 \mathrm{~mm}$ deep, consisted of eight straight segments that were connected by right-angled corners and formed a path with a total length of $260 \mathrm{~mm}$. At each corner, a so-called 'dead-end' was included to enforce a choice with regard to the direction in which to proceed. Additionally, the maze path always constituted a closed loop and could therefore be traced continuously during a given period of time. The different maze trajectories are depicted in Figure 1 as part of an overview on the study's design described in more detail below.

Subjects were instructed to trace the maze path as fast and as accurately as possible, while keeping their eyes closed throughout the whole experiment. Only the dominant right hand was used and the tracing movements had to be performed in a clockwise fashion. The instructions also stressed that subjects should make an effort to learn the executed movement patterns, in order to be able to reproduce the established motor routines later on without having to rely on the maze's groove to guide them.

Since becoming more proficient at executing the required movement sequences entailed an increase in motor ouput (i.e., velocity) over time, two additional control conditions were included to distinguish purely performance-related changes in brain activity from true learning-related effects (van Mier et al., 
1998). Both control conditions consisted of tracing a square trajectory, which all subjects were familiar with because it was shortly traced during the adjustment of the setup prior to scanning (see below). In the control conditions, the square trajectory should either be traced as fast as possible ('SQfast'), or at a velocity matching the slow tracing at the early stages of learning ('SQslow'), assuming that no additional sequence-specific learning would occur.

Both the subjects' head and arms were stabilized by foam padding to minimize task-related motion. The necessary tracing movements were mainly performed using the joint of the wrist. The mazes were fixated in a specifically designed holder, which was placed on a custom-built table that covered the subject's abdomen. Before starting the experiment, the position of the table was adjusted in such a way that the required movements could be performed rather effortlessly. A resistance-based MR-compatible recording method was used to register the pen tracing movements while scanning (Reithler et al., 2006).

\subsubsection{Order and Structure of Experiments}

All twelve subjects participated in a series of experiments. During the first scanning session (scheduled on Monday), they had to learn two new movement sequences by continuously tracing one of two mazes in blocks of 30 seconds. The active tracing blocks were alternated with rest periods (lasting for 20 seconds) in which the pen should be kept at a fixed starting position, to which the subject's hand was re-positioned at the end of each tracing block by the experimenter. The start and end of each tracing block were indicated by a short high $(1000 \mathrm{~Hz})$ and low $(500 \mathrm{~Hz})$ tone, respectively. The same maze was traced for twelve consecutive blocks, leading to an effective overall training duration of 6 minutes. In addition, the two square control conditions were presented in an interleaved fashion with respect to the two learning conditions (see Figure 1a). Both control conditions consisted of six $30 \mathrm{~s}$ blocks. In between the different tracing conditions, a $30 \mathrm{~s}$ 'switch' period was introduced in which the experimenter interchanged the mazes/squares according to the experimental protocol. The condition order was counterbalanced across subjects.

On each following day, subjects repeatedly practiced one of the two mazes of the first scanning session more extensively during an additional set of twelve 30 $\mathrm{s}$ blocks (Figure 1b). These additional practice sessions were performed in a mock scanner, to ensure that the subjects would perform the required movements while lying in the same position as during the real scanning sessions. The specific maze which was extensively trained was varied across subjects, so that the sequence which was repeatedly practiced by six subjects functioned as the non-overlearned reference sequence for the other six subjects and vice versa. 
During the second scanning session (scheduled on Friday), there were three mazes which had to be traced following a similar scheme as in scanning session one (Figure 1c). The mazes differed with respect to the amount of practice that had been invested in executing the corresponding movement sequence so far. With one maze, subjects were familiar because they had traced it in the first scanning session. However, no additional efforts were made to practice this particular sequence afterwards ('OLD'). Another maze was the one they had been practicing on a daily basis, in a total of 48 practice blocks across four days, and which therefore was considered as overlearned ('OVER'). The final maze involved a trajectory which was completely new to the subjects ('NEW'). The six possible orders in which the three conditions could be presented were counterbalanced across subjects.

To exclude the possibility that subjects merely traced along the mazes' borders without actively learning the new movement patterns, they were asked to reproduce the learned movement sequence by drawing the mazes by heart after each scanning- and practice-session. This 'free drawing' was performed using paper and pencil, while subjects still lay in the scanner and kept their eyes closed. 
a)

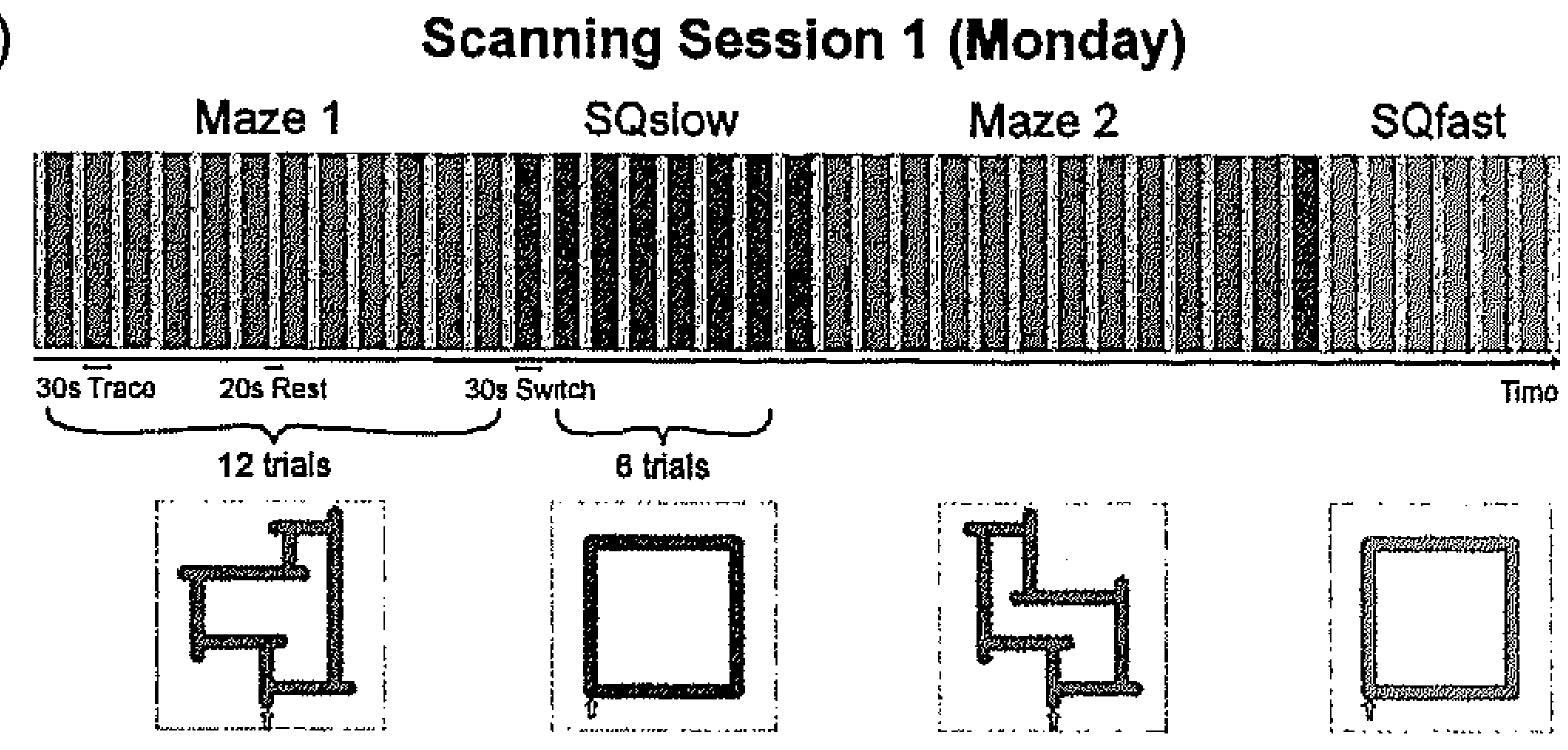

b)

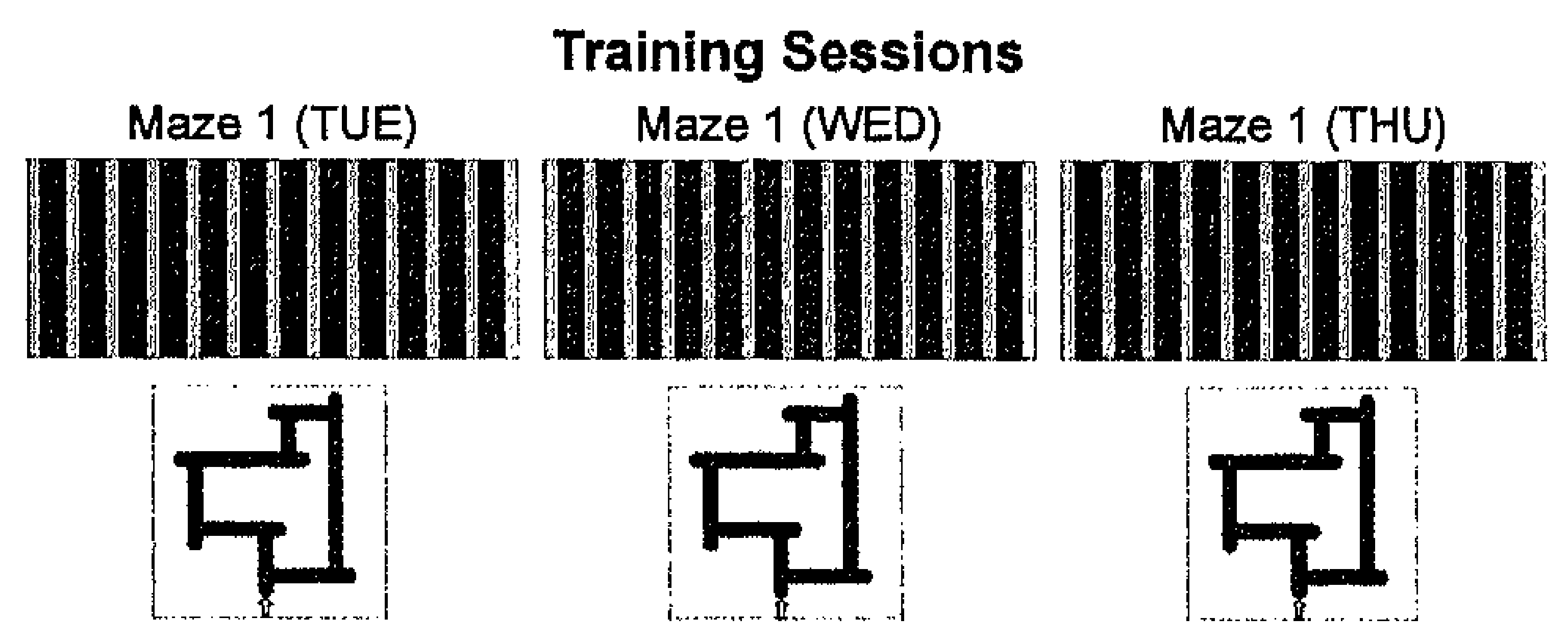

c)

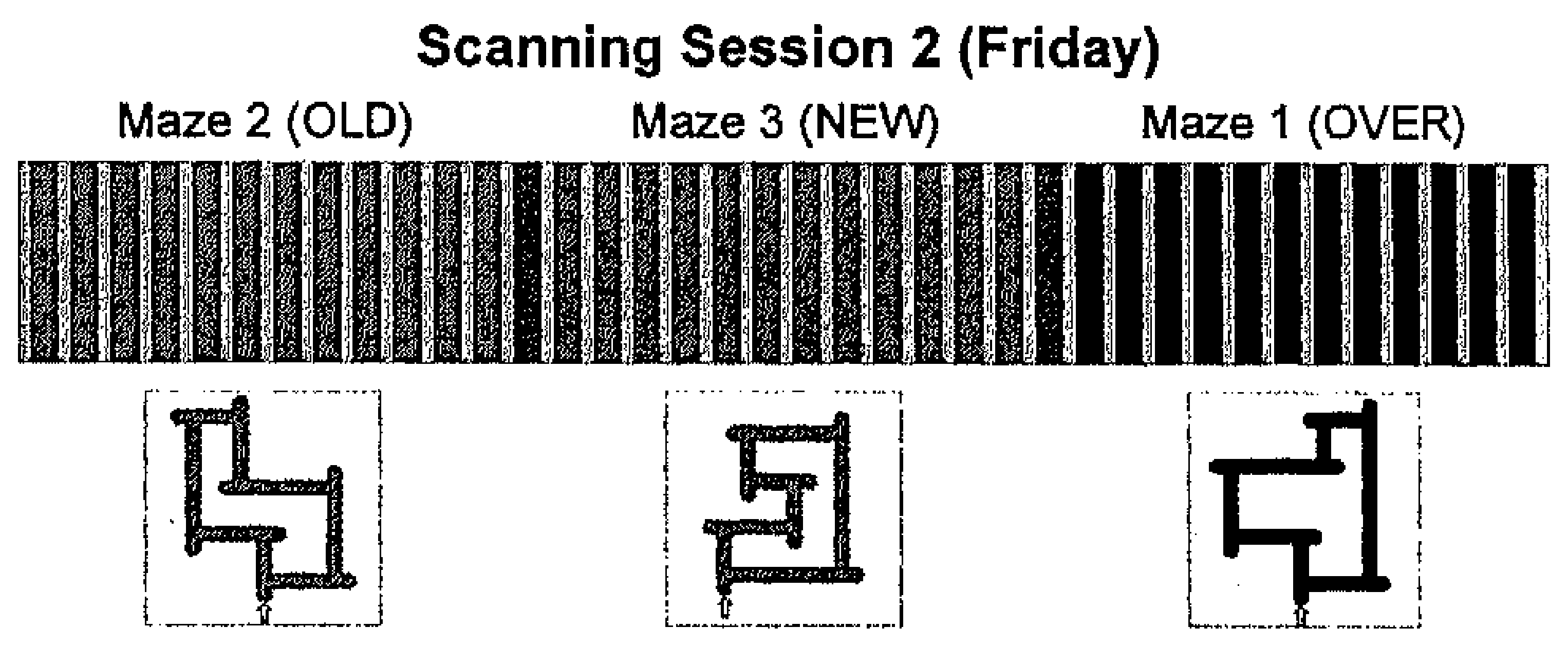

Figure 1. Overview of the experimental design. a) The maze trajectories depicted here represent the new movement sequences that subjects had to learn during the first scanning session. The square trajectories were included as control conditions (see Materials and Methods for details). Tracing was continuously performed for $30 \mathrm{~s}$ during 12 (mazes) or 6 (squares) consecutive trials, alternated with resting periods (lasting $20 \mathrm{~s}$ ). Note that the small white arrows at the bottom indicate the starting position for each trajectory. b) On the three days between both fMRI measurements, additional training sessions were scheduled during which one of the previously performed sequences was extensively practiced. c) During the second scanning session, three different mazes needed to be traced: the two mazes from session 1 (of which one was extensively trained in the meantime ('OVER'), whereas the other was not ('OLD')), and a completely novel maze ('NEW'). Note that which maze was overlearned was varied across subjects, and the order in which the different conditions were presented was counterbalanced for both sessions. Color coding of experimental conditions included in fMRI sessions: green indicates that subjects did not have any prior experience performing a given sequence; blue colors represent an overlearned status; red denotes that the corresponding sequence was performed before, but was not extensively trained. 


\subsection{Image Acquisition}

Imaging data were collected on a 3T Siemens Magnetom Allegra head-scanner (Siemens Medical Systems, Erlangen, Germany) via a regular volume coil. Both scanning sessions started with the acquisition of an anatomical 3D dataset consisting of 176 slices (MDEFT sequence (Deichmann et al., 2004); Repetition Time $(\mathrm{TR})=7.92 \mathrm{~ms}$; Echo Time $(\mathrm{TE})=2.4 \mathrm{~ms}$; Flip Angle $=15^{\circ}$, voxel dimensions $=1 \times 1 \times 1 \mathrm{~mm}^{3}$; Field of View $($ FoV $)=256 \times 224 \mathrm{~mm}^{2}$ ). Subsequently, 1576 functional volumes comprising 21 transversal slices were recorded using a gradient-echo echo-planar imaging sequence $(\mathrm{TR}=1250 \mathrm{~ms}$; $\mathrm{TE}=28 \mathrm{~ms} ;$ Flip Angle $=67^{\circ}$; voxel dimensions $=3.5 \times 3.5 \times 5 \mathrm{~mm}^{3} ;$ gap $=1$ $\mathrm{mm} ; \mathrm{FoV}=224 \times 224 \mathrm{~mm}^{2}$ ), resulting in whole-brain coverage. Functional data were collected in a single continuous run in order to minimize scanner-related changes in the overall signal level across conditions.

\subsection{Data Analysis}

The acquired behavioral data were analyzed using in-house software written in MATLAB (v6.5 R13; The Mathworks, Natick, USA). Because of the high sampling rate at which the behavioral data were collected $(\sim 666 \mathrm{~Hz}$; for details see Reithler et al., 2006), a TR-based binning could be used while extracting the most relevant performance measures: the mean velocity achieved, the percentage of samples being part of a stop, and finally the percentage of samples constituting a retrace. A stop was assigned whenever the tracing velocity was lower than $10 \mathrm{~mm} / \mathrm{s}$ during $100 \mathrm{~ms}$ or more. A retrace consisted of at least 150 consecutive samples which were traced in the wrong (i.e., counterclockwise) direction. Separate repeated measures Analyses of Variance (ANOVAs) were computed for all three performance measures to reveal how learning evolved within and across tracing conditions.

All imaging data were analyzed using BrainVoyager QX (v1.7; Brain Innovation, Maastricht, The Netherlands). The different analysis steps are described in detail below.

\subsubsection{Anatomical Data}

The acquired anatomical datasets from both MR sessions were first corrected for spatial intensity inhomogeneities by estimating a bias field based on a preliminary white matter segmentation (Vaughan et al., 2001). For each subject, the dataset from the first scanning session was subsequently brought into ACPC space. Next, the datasets from both sessions were aligned using the ACPC version of the first dataset as the target volume and were separately transformed into standard Talairach space (Talairach and Tournoux, 1988). All performed transformations were then re-applied in a single step using sinc interpolation 
(Goebel et al., 2006) and both datasets were averaged. To optimize the 2D-3D alignment, the surrounding head tissue was removed using an automatic 'brain peeling' tool.

An additional processing step involved the semi-automatic segmentation of the grey and white matter boundary (Kriegeskorte and Goebel, 2001), followed by an inflation of the cortical surface. Finally, a cortex-based inter-subject alignment was separately performed for each hemisphere based on the individual brains' curvature information and using a moving target group averaging approach (Goebel et al., 2006). After the inter-subject alignment, a folded average cortex representation was constructed for both hemispheres, on which the statistical maps on the group level will be projected.

\subsubsection{Functional Data}

Preprocessing of the individual datasets followed standard procedures optimized for the current application, and included the following steps: first, a slice scan time correction was performed using sinc interpolation to correct for the sequentially executed interleaved slice acquisition. Next, detection (trilinear interpolation) and correction (sinc interpolation) of 3D motion was performed to spatially align all functional volumes (of both sessions) to a single volume in the middle of the first scanning session using rigid-body transformations. Although the four initial volumes were excluded from further statistical analyses due to their stronger T1 saturation, the first volume was nevertheless coregistered to the middle volume in order to function as a reference with high anatomical detail during the intensity-driven fine tuning of the 2D-3D alignment later on. Finally, linear trend removal and high-pass filtering with a cut-off of 12 cycles per time course $(0.0061 \mathrm{~Hz})$ were performed to remove low frequency drifts. No spatial smoothing was applied.

The functional data were interpolated to a $3 \times 3 \times 3 \mathrm{~mm}^{3}$ voxel target resolution. In the context of the cortex-based inter-subject alignment procedure described in the previous section, sampling of the functional data was restricted to grey matter voxels based on the anatomical information from the individual cortex segmentations. The statistical analyses were carried out using a voxel-wise General Linear Model (GLM) at the single subject level, based on design matrices which included the estimated $3 \mathrm{D}$ motion parameters obtained during preprocessing as well as predictors for all relevant task conditions. An additional predictor was included to model the repositioning of the hand at the end of each tracing trial. Condition effects were modeled using a boxcar function which was convolved with a theoretical Two Gamma hemodynamic response function. The reported group analyses were conducted following a random effects model. Unless stated otherwise, statistical maps on the group level were thresholded at $t=3.15$ with a corresponding probability of $p$ (uncorr) $<0.009$. More detailed analyses were additionally performed for functionally 
Chapter 3

defined Regions-of-Interest (ROIs) by running separate random effects ROIGLMs.

\section{Results}

\subsection{Scanning Session 1 (Day 1)}

\subsubsection{Behavioral Data}

The registered pen tracing data were summarized and analyzed across subjects at the level of single trials. To identify potential effects of learning within a given condition, the first and last trial of that condition were contrasted as they constituted the two extremes with respect to the amount of preceding practice.

First, a two-way repeated measures ANOVA with Maze (2 levels) and Trial (2 levels: 1 vs. 12) as within-subject factors was performed for all three performance measures. Significant 'Maze $\mathrm{x}$ Trial' interactions were found for velocity $\left(F_{(1,11)}=5.66 ; p=0.037\right)$ and stops $\left(F_{(1,11)}=9.56 ; p=0.010\right)$, whereas only significant main effects of Maze $\left(F_{(1,11)}=10.03 ; p=0.009\right)$ and Trial $\left(F_{(1,11)}\right.$ $=11.565 ; \mathrm{p}=0.006)$ were observed for retraces. As can be seen in Figure 2 (which shows the min-max normalized performance on the group level for all 3 behavioral measures), there was a significant increase in velocity accompanied by a decrease in the percentage of stops and retraces. For the first maze, the mean velocity increased from 17 to $45 \mathrm{~mm} / \mathrm{s}\left(\mathrm{F}_{(1,11)}=34,16 ; \mathrm{p}=0.000\right)$, and the stop and retrace percentages dropped from 53 to $26 \%$ and from 13 to $3 \%$, respectively (stops: $F_{(1,11)}=29.79 ; p=0.000$; retraces: $F_{(1,11)}=7.38 ; p=0.020$ ). A similar pattern of results was obtained for the second maze, although the level of the initial performance was higher compared to the first maze: the mean velocity increased from 30 to $51 \mathrm{~mm} / \mathrm{s}$ when comparing the first to the last trial $\left(F_{(1,11)}=22.73 ; \mathrm{p}=0.001\right)$, while a decrease from 35 to $23 \%$ was found for the stop percentages $\left(\mathrm{F}_{(1,11)}=8.58 ; \mathrm{p}=0.014\right)$. There was only a trend with respect to a decrease in the retrace percentages (from 4 to $2 \% ; \mathrm{F}_{(1,11)}=4.74 ; \mathrm{p}=0.052$ ). Finally, the mean velocity achieved during the square tracing was $28 \mathrm{~mm} / \mathrm{s}$ (standard error $(\mathrm{se})=1.5$ ) for the slow, and $68 \mathrm{~mm} / \mathrm{s}(\mathrm{se}=2.4$ ) for the fast tracing condition, indicating that subjects followed the instructions for these control conditions correctly. 


\section{a)}
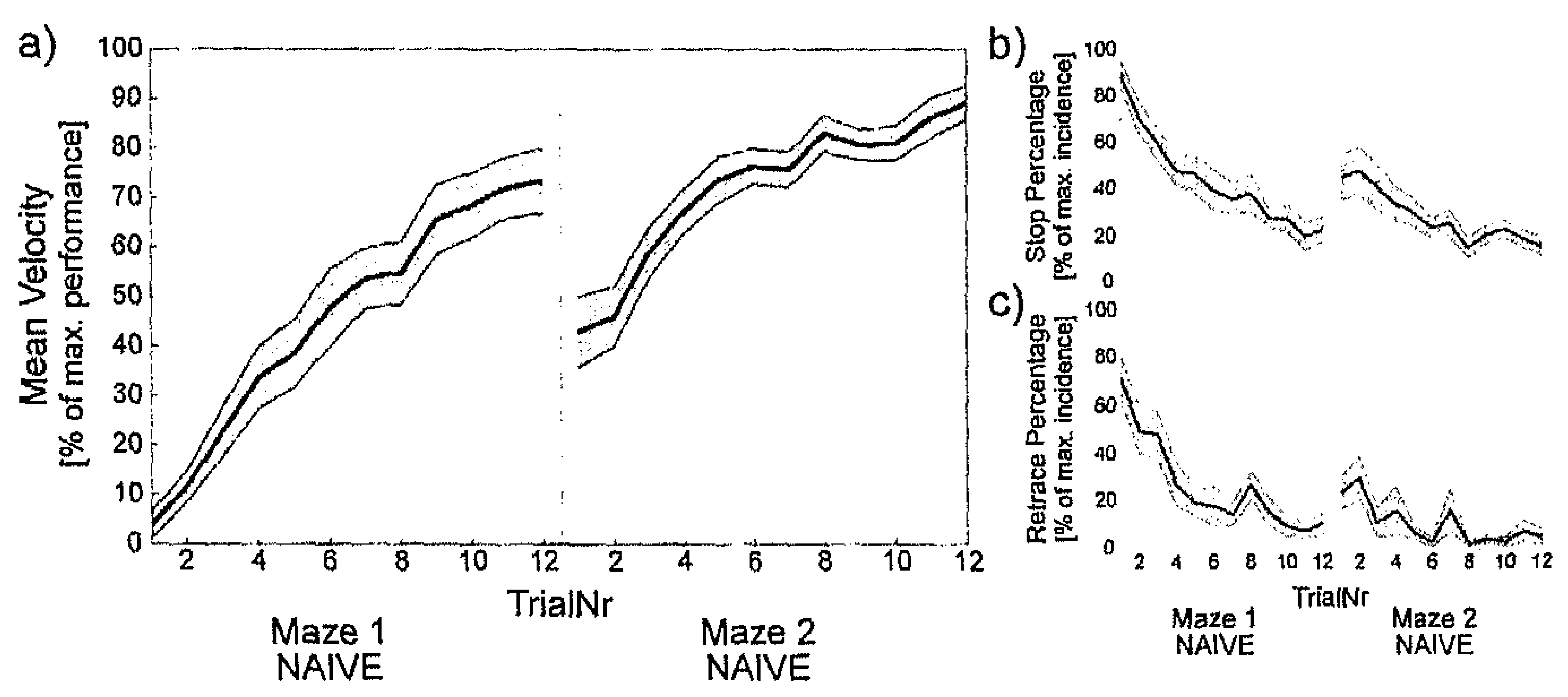

Figure 2. Summary of the behavioral data obtained in scanning session 1. Panel a) shows the increase in tracing velocity achieved across the 12 trials of both maze conditions, which is accompanied by a decrease in the stop and retrace percentages (depicted in part $b$ and $c$, respectively). The figures represent the min-max normalized performance for the 3 performance measures on the group level across all 24 trials. The shaded regions indicate the standard errors for the obtained trial means. The classification 'NAIVE' is used here to indicate that both the task itself and the traced mazes were completely new to the subjects (note that the label ' $N E W$ ' is only used in the context of the second fMRI session later on).

\subsubsection{Imaging Data}

In order to identify brain areas which were activated in all four tracing conditions of the first scanning session (i.e., irrespective of which movement sequence was performed), a contrast involving a conjunction across conditions versus baseline was calculated. As shown in Figure 3a, the main cluster of activation was located within the left central sulcus, corresponding to the hand/wrist position within the somatotopic map of the primary motor (M1) and sensory (S1) cortices (Lotze et al., 2000; Alkadhi et al., 2002). The extent of this activation cluster further encompassed both the anterior bank of the precentral gyrus and part of the postcentral sulcus. Additionally, activations were observed bilaterally in the supplementary motor area (SMA) and the inferior postcentral sulcus. At the subcortical level, only unilateral activations emerged in the left putamen and thalamus, and the ipsilateral cerebellum (not shown). These activations represent the basic network of areas associated with the execution of the required movements per se, irrespective of the specific trajectory or the level of proficiency. When performing the same contrast without including the square control conditions, the extent of the previously identified cortical network increased to encompass additional (bilaterally emerging) regions showing enhanced activation during the tracing of both mazes compared to baseline (Figure 3b). 


\section{Chapter 3}

Because the movements in the current task were not externally paced, the possibility that learning-related changes in activity were confounded by corresponding changes in movement velocity needed to be ruled out. An additional contrast between the SQfast and SQslow conditions revealed that the previously mentioned left M1/S1 and the ipsilateral cerebellar foci were the only regions to show strict velocity-dependent responses as indicated by their significantly stronger response during the fast tracing condition. There were no regions which responded more strongly to the SQslow condition.

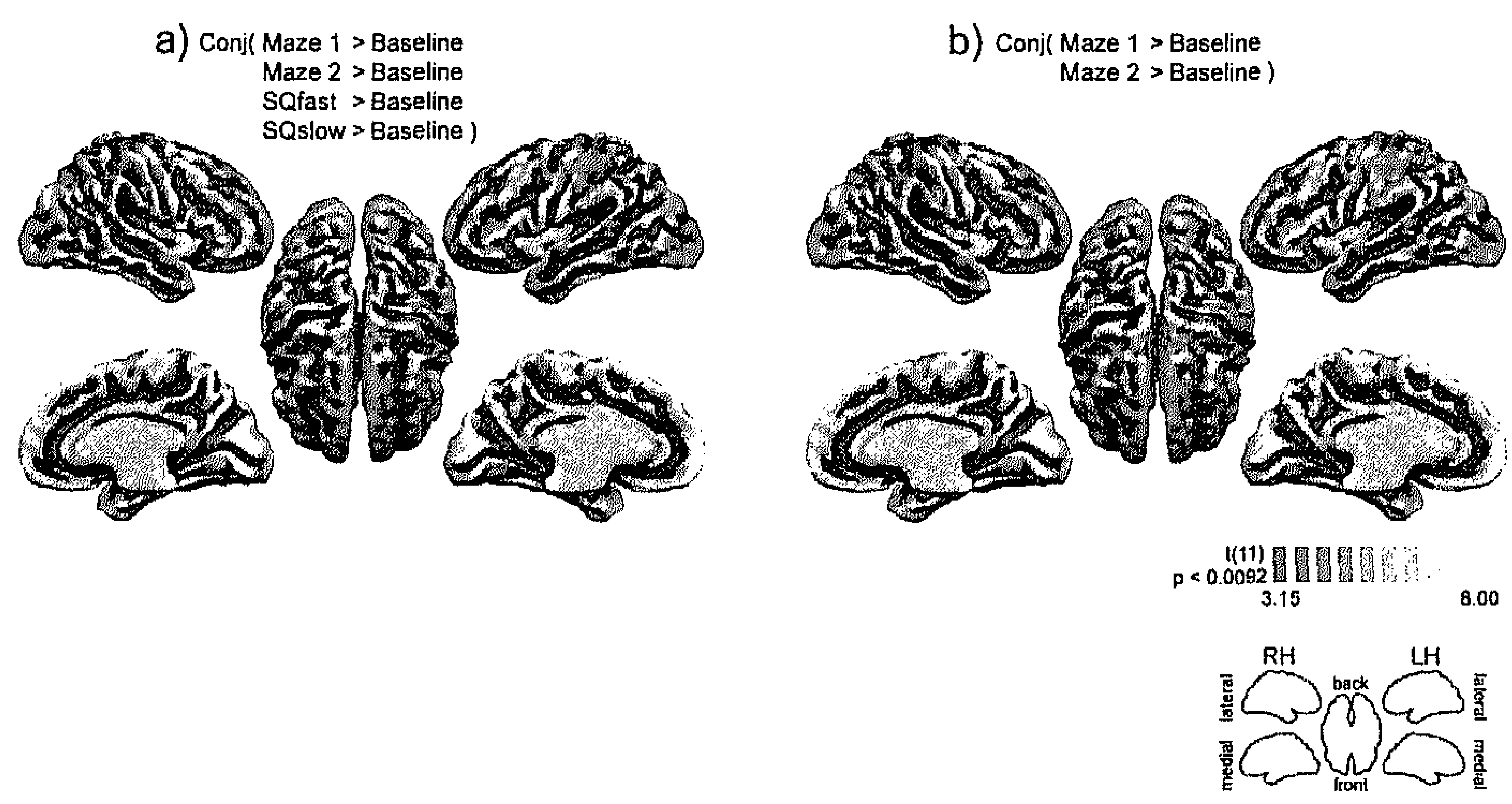

Figure 3. General tracing-related brain activations (Session 1), projected on the average group cortex mesh. a) Regions that were activated during all tracing conditions, irrespective of which trajectory was traced or the velocity at which the tracing was performed. b) Areas showing increased responses when the same contrast was performed without including the square control conditions, i.e. during the tracing of the mazes (as compared to baseline). The color scale represents the significance level of the identified activations (varying from $t=3.15$ to $t=8.00$ ). Abbreviations: $\mathrm{RH}$, right hemisphere; $\mathrm{LH}$, left hemisphere.

Based on the assumption that learning-related areas should be maximally engaged during the acquisition of the new movement sequences, a contrast between the two maze tracing and the two square conditions was computed (Figure 4a). This resulted in the identification of a 'learning' network consisting of the following regions: the bilateral superior precentral sulcus at the level of the most caudal part of the superior frontal sulcus (dorsal premotor cortex, PMd), the bilateral anterior bank of the inferior precentral gyrus (ventral premotor cortex, PMv), the bilateral inferior postcentral sulcus (InfPCS), the bilateral anterior intraparietal sulcus (aIPS), the bilateral posterior superior parietal lobule (SPL), and a region in the left medial IPS/posterior SPL (the corresponding Talairach coordinates are listed in Table 1). Besides showing 
markedly stronger responses in both learning conditions, these regions did not discriminate between the fast and the slow execution of the control sequences, suggesting their responses are not velocity-dependent (Figure $4 \mathrm{~b}$ ).

a)

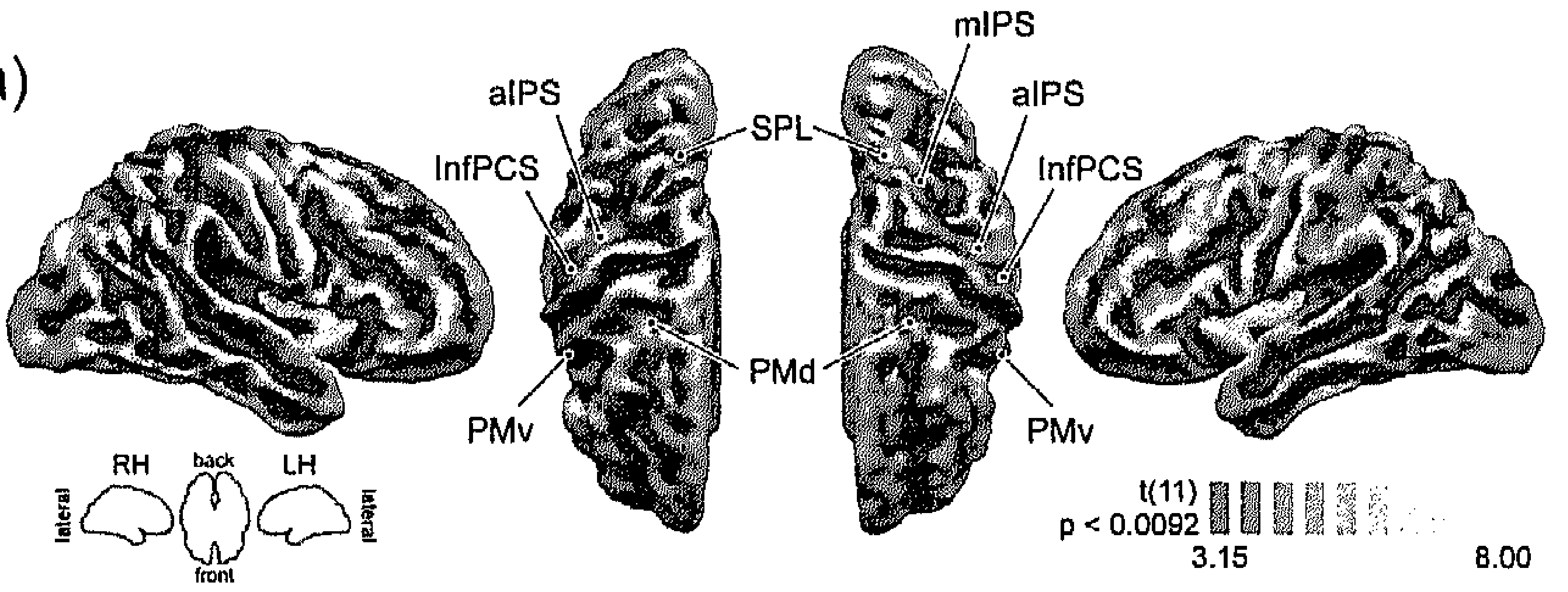

b)

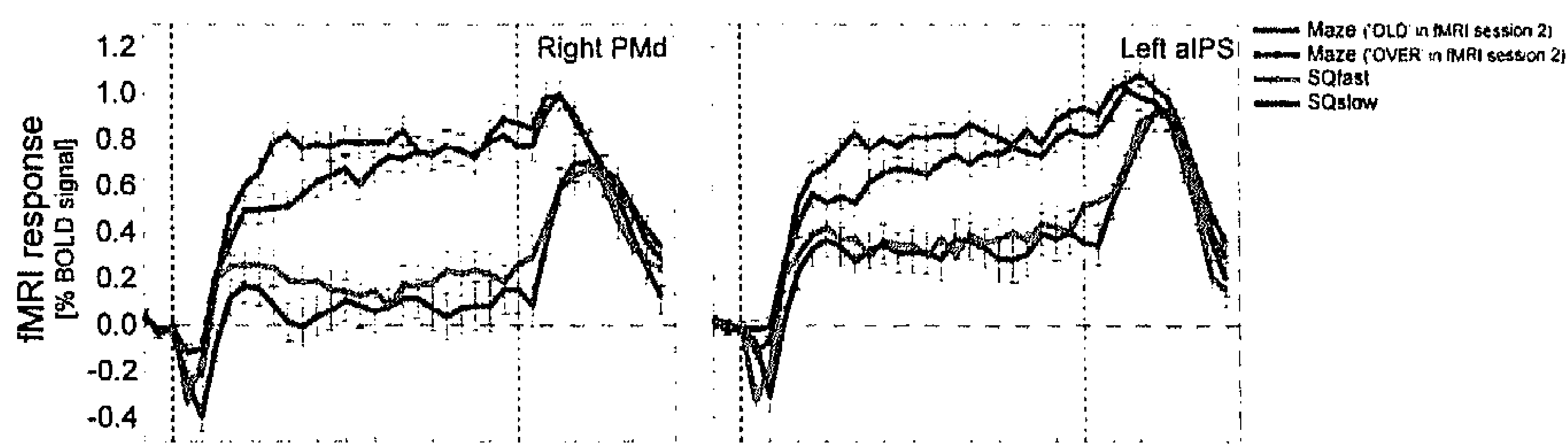

c)

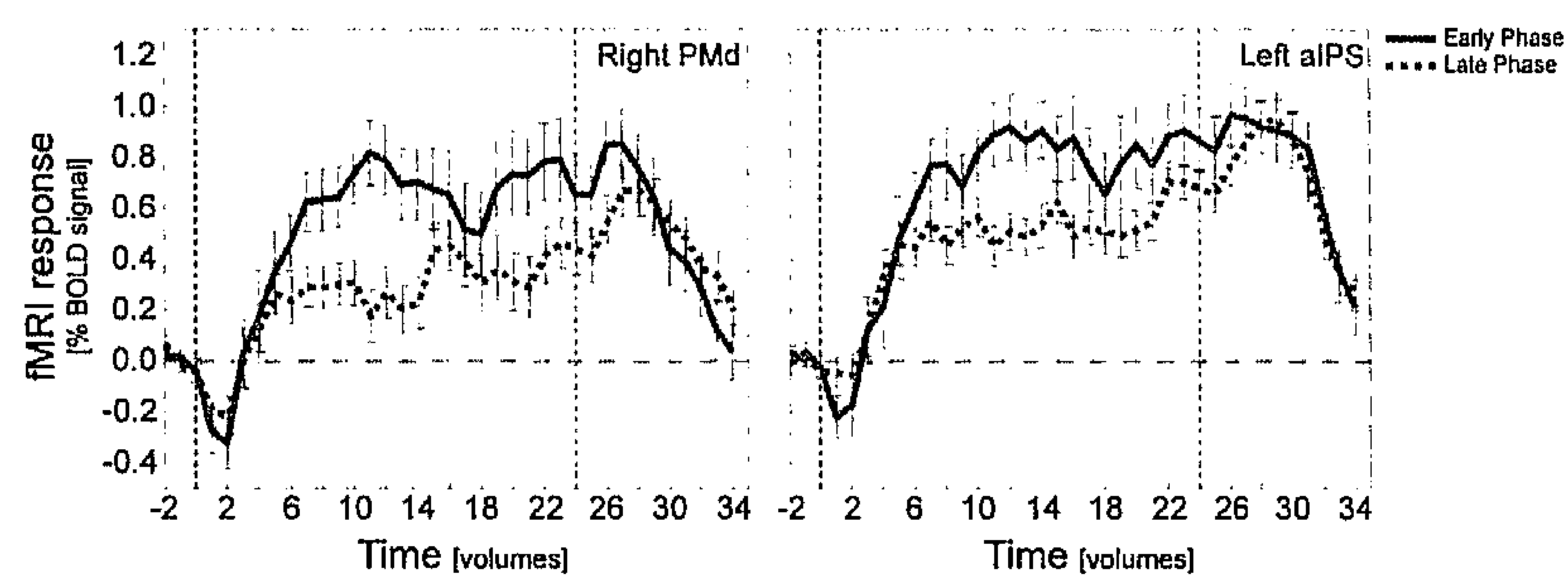

Figure 4. Learning-related brain activations (Session 1). a) The bilateral network of areas showing specific learning-related activations, viewed from the lateral sides as well as from the top. b) Event-related averages of the responses to the different tracing conditions in session 1 (averaged across subjects), extracted from the right PMd and left aIPS ROIs. Note that the response to both maze tracing conditions is stronger than the response to either of the control conditions. c) Responses during the early and late stage of maze 2 extracted from the same ROIs, reflecting the decrease in activation as found across the identified network associated with an increase in proficiency. The vertical dashed lines indicate the start and end of the active tracing period. Time is expressed in volumes, the time it took to acquire the 21 slices which covered the subjects' brains once (one volume corresponds to $1250 \mathrm{~ms}$ ). 
Abbreviations: PMd, dorsal premotor cortex; PMv, ventral premotor cortex; InfPCS, inferior postcentral sulcus; aIPS, anterior intraparietal sulcus; mIPS, medial intraparietal sulcus; SPL, superior parietal lobule; RH, right hemisphere; LH, left hemisphere.

To further characterize the involvement of the aforementioned areas throughout the course of learning, both maze tracing conditions were divided into an early and a late phase (similar to e.g. Floyer-Lea and Matthews, 2004). This categorization was based on the min-max normalized learning curve of the achieved mean velocity, extracted from the individual subject's behavioral data. Trials in which less than $25 \%$ of the maximum performance level was reached were classified as belonging to the early learning phase. The trials at the end of the maze tracing conditions in which a performance of $75-100 \%$ was achieved, were included in the late phase. Moreover, for each subject at least two trials were included in either phase to ensure a stable estimation of the individual activation levels. The data from two subjects were excluded because their behavioral learning curves did not allow a clear distinction between learning phases. When using the previously designated 'learning-related' areas as ROIs, the following pattern of results was obtained when contrasting the learning phases for both mazes (see Table 1): whereas the activation in the right PMd, $\mathrm{PMv}$, the bilateral inferior postcentral sulcus, and the left aIPS started to decrease over time while learning maze 1, even stronger decreases in activation were observed for almost all ROIs when comparing the two learning stages for the second maze. Interestingly, the only ROIs which did not show a similar activation pattern were located in the posterior SPL (bilaterally). The more modest (or even absent) changes in activation level during the acquisition of the first sequence might point to the continued involvement of the identified regions in learning on a meta-level, in other words related to the general task requirements as opposed to learning processes which are specific for the executed sequence itself. This is also reflected in the behavioral data by an apparent cross-over effect (better performance for maze 2 than maze 1 when comparing the first trials). To get an impression of what happened throughout the entire first scanning session, the late phase of the second maze (at the end of the session) was compared to the early phase of the first maze (at the beginning of the session). The only region which did not show a decrease in activation level across mazes was the left posterior SPL. Finally, a contrast between the two control conditions was re-computed at the ROI-level. The results confirmed that the activation changes associated with learning were not confounded by general increases in movement velocity (except potentially for the left InfPCS activation, see last column Table 1).

To ensure that no interesting activation changes were missed by applying a ROI-based approach, the early and late phase of both maze tracing conditions were contrasted at the whole-brain level. The resulting maps supported the 
earlier observation that the decreases in activation in the bilateral InfPCS and aIPS, as well as (predominantly right) dorsal and ventral premotor cortices, were more pronounced for the second maze. Additionally, a similar decrease in activation while learning the second sequence was observed in the bilateral SMA (Talairach coordinates [x,y,z; in mm]: -4, -7, 60 [left]; 7, -6, 58 [right]).

\begin{tabular}{|c|c|c|c|c|c|c|c|c|c|c|c|c|}
\hline \multirow{3}{*}{$\begin{array}{l}\text { Region } \\
\text { of } \\
\text { Interest }\end{array}$} & \multirow[t]{3}{*}{$\begin{array}{l}\text { Hemi- } \\
\text { sphere }\end{array}$} & \multirow{2}{*}{\multicolumn{3}{|c|}{$\begin{array}{l}\text { Talairach } \\
\text { Coordinates } \\
\text { [mm] }\end{array}$}} & \multicolumn{8}{|c|}{$\begin{array}{l}\text { Significance of Activation Changes } \\
\text { t-values and probabilities for contrasts (RFX ROI-GLM; } n=10 \text { ) }\end{array}$} \\
\hline & & & & & $\begin{array}{c}\text { Maze I } \\
\text { Early }\end{array}$ & $>\underset{\text { Late }}{\text { Maze } 1}$ & $\underset{\text { Early }}{\text { Maze } 2}$ & $>\underset{\text { Late }}{\text { Maze } 2}$ & $\begin{array}{l}\text { Maze } \\
\text { Early }\end{array}$ & $>\underset{\text { Late }}{\text { Maze } 2}$ & $\begin{array}{c}\text { Square } \\
\text { Fast }\end{array}$ & $>$ Square \\
\hline & & $\mathrm{x}$ & $y$ & $z$ & $\mathrm{t}$ & (p) & $t$ & (p) & $t$ & (p) & $\mathrm{t}$ & (p) \\
\hline PMd & $\begin{array}{l}\mathrm{LH} \\
\mathrm{RHI}\end{array}$ & $\begin{array}{r}-21 \\
25\end{array}$ & $\begin{array}{l}-9 \\
-8\end{array}$ & $\begin{array}{l}52 \\
48\end{array}$ & $\begin{array}{l}2.18 \\
3.03\end{array}$ & $\begin{array}{l}(0.057) \\
\left(0.0[4)^{*}\right.\end{array}$ & $\begin{array}{l}3.30 \\
5.39\end{array}$ & $\begin{array}{l}(0.009)^{*} \\
(0.000)^{* *}\end{array}$ & $\begin{array}{l}4.15 \\
7.11\end{array}$ & $\begin{array}{l}(0.003)^{k *} \\
(0,000)^{* *}\end{array}$ & $\begin{array}{l}1.34 \\
0.67\end{array}$ & $\begin{array}{l}(0.213) \\
(0.523)\end{array}$ \\
\hline PMv & $\begin{array}{l}\text { L.H } \\
\text { RH }\end{array}$ & $\begin{array}{r}-52 \\
52\end{array}$ & $\begin{array}{r}-1 \\
2\end{array}$ & $\begin{array}{l}34 \\
34\end{array}$ & $\begin{array}{l}1.37 \\
3.08\end{array}$ & $\begin{array}{l}(0.204) \\
(0.013)^{*}\end{array}$ & $\begin{array}{l}3.50 \\
7.73\end{array}$ & $\begin{array}{l}(0.007)^{*} \\
(0.000)^{* *}\end{array}$ & $\begin{array}{l}2.87 \\
5.46\end{array}$ & $\begin{array}{l}(0.019)^{*} \\
(0.000)^{* *}\end{array}$ & $\begin{array}{l}-0.74 \\
-1.47\end{array}$ & $\begin{array}{l}(0.476) \\
(0.177)\end{array}$ \\
\hline InfPCS & $\begin{array}{l}\mathrm{LH} \\
\text { RH }\end{array}$ & $\begin{array}{r}-54 \\
49\end{array}$ & $\begin{array}{l}-21 \\
-24\end{array}$ & $\begin{array}{l}32 \\
37\end{array}$ & $\begin{array}{l}3.19 \\
4.34\end{array}$ & $\begin{array}{l}(0.011)^{*} \\
(0.002)^{* *}\end{array}$ & $\begin{array}{l}4.20 \\
4.89\end{array}$ & $\begin{array}{l}(0.002)^{* *} \\
(0.001)^{* *}\end{array}$ & $\begin{array}{l}5.90 \\
6.01\end{array}$ & $\begin{array}{l}(0.000)^{* *} \\
(0.000)^{* *}\end{array}$ & $\begin{array}{l}2.81 \\
1.44\end{array}$ & $\begin{array}{l}(0.021)^{*} \\
(0.184)\end{array}$ \\
\hline alps & $\begin{array}{l}\mathrm{LH} \\
\mathrm{RH}\end{array}$ & $\begin{aligned}-39 \\
36\end{aligned}$ & -33 & $\begin{array}{l}42 \\
39\end{array}$ & $\begin{array}{l}2.37 \\
2.01\end{array}$ & $\begin{array}{l}(0.042)^{*} \\
(0.076)\end{array}$ & $\begin{array}{l}5.58 \\
3.43\end{array}$ & $\begin{array}{l}(0.000)^{* *} \\
(0.008)^{*}\end{array}$ & $\begin{array}{l}4.59 \\
4.90\end{array}$ & $\begin{array}{l}(0.001)^{* *} \\
(0.001)^{* *}\end{array}$ & $\begin{array}{r}1.12 \\
-0.09\end{array}$ & $\begin{array}{l}(0.294) \\
(0.932)\end{array}$ \\
\hline mIPS & $\begin{array}{l}\mathrm{LH} \\
\mathrm{RH}\end{array}$ & -26 & -53 & 51 & 0.20 & $(0.844)$ & 3.12 & $(0.012)^{*}$ & 2.42 & $(0,039)^{*}$ & -0.48 & $(0.646)$ \\
\hline SPL & $\begin{array}{l}\mathrm{LH} \\
\text { RH }\end{array}$ & $\begin{array}{r}-17 \\
13\end{array}$ & $\begin{array}{l}-66 \\
-59\end{array}$ & $\begin{array}{l}52 \\
53\end{array}$ & $\begin{array}{r}-1.00 \\
0.08\end{array}$ & $\begin{array}{r}(0.344) \\
(0.940)\end{array}$ & $\begin{array}{l}1.84 \\
2.13\end{array}$ & $\begin{array}{l}(0.098) \\
(0.062)\end{array}$ & $\begin{array}{r}-0.12 \\
2.37\end{array}$ & $\begin{array}{l}(0.904) \\
(0.042)^{*}\end{array}$ & $\begin{array}{l}-1.75 \\
-1.99\end{array}$ & $\begin{array}{l}(0.114) \\
(0.078)\end{array}$ \\
\hline
\end{tabular}

Table 1. Results of the ROI analyses on the changes in activation level depending on the stage of (intra-session) learning, for the learning-related ROIs as defined in scanning session 1 .

${ }^{*} \mathrm{p}<0.05$ (uncorrected); ${ }^{* *} \mathrm{p}<0.0045$ (Bonferroni-corrected for the number of included ROIs). Abbreviations: PMd, dorsal premotor cortex; $\mathrm{PMv}$, ventral premotor cortex; InfPCS, inferior postcentral sulcus; aIPS, anterior intraparietal sulcus; mIPS, medial intraparietal sulcus; SPL, superior parietal lobule; LH, left hemisphere; RH, right hemisphere.

\subsection{Training Sessions (Days 2-4)}

A two-way repeated measures ANOVA of the behavioral data gathered on the days in between the two scanning sessions with Session (Tuesday, Wednesday and Thursday) and Trial (trial numbers 1 and 12) as within-subject factors, revealed the following results: A significant interaction between Session and Trial emerged for the achieved velocity $\left(\mathrm{F}_{(2,22)}=3.97 ; \mathrm{p}=0.034\right)$, and significant main effects for both factors were found for the stop percentages (Session: $F_{(2,22)}=5.21 ; p=0.014 ;$ Trial: $F_{(2,22)}=12.80 ; p=0.004$ ). The retrace percentages did not show such effects. When comparing the first and last trial of each training session separately, a significant increase in velocity from 53 to 71 
$\mathrm{mm} / \mathrm{s}\left(\mathrm{F}_{(1,11)}=19.91 ; \mathrm{p}=0.001\right)$ and a decrease in the stop percentage from 21 to $16 \%\left(\mathrm{~F}_{(1,11)}=5.20 ; \mathrm{p}=0.043\right)$ were only found for the first training session (Tuesday). For the other two training sessions, no additional changes were detected for any of the behavioral measures, suggesting that a stable performance level was reached. After completing the three training sessions, the mean velocity achieved was $76 \mathrm{~mm} / \mathrm{s}(\mathrm{se}=7.4)$, while the stop and retrace percentages were $13 \%(\mathrm{se}=3.1)$ and $0.2 \%(\mathrm{se}=0.1)$, respectively.

Although not analyzed in a quantitative manner, the free drawings that were produced after each training session furthermore suggest that the representation of the executed movement sequence became more precise. Both the general layout of the maze and the relative proportions between its elements were generally reproduced more accurately as learning progressed.

\subsection{Scanning Session 2 (Day 5)}

\subsubsection{Behavioral Data}

Three different maze tracing conditions were included in the second scanning session, allowing to contrast the performance at different levels of proficiency. A two-way repeated measures ANOVA with Condition (overlearned, old and new) and Trial (1 vs. 12) as within-subjects factors revealed significant 'Condition by Trial' interactions for velocity $\left(\mathrm{F}_{(2,22)}=6.55 ; \mathrm{p}=0.006\right)$, as well as stops $\left(\mathrm{F}_{(2,22)}=16.62 ; \mathrm{p}=0.000\right)$, whereas for the retrace percentage, only a significant main effect for Trial was found $\left(F_{(1,11)}=7.244 ; p=0.021\right)$. Next, the first and last trials of the three tracing conditions were contrasted separately. Because performance on the overlearned sequence reached an asymptotic level during the previous training sessions, no additional changes were expected to occur while subjects traced the extensively trained sequence again in the second scanning session ('OVER'). This was indeed the case for both the stop $\left(\mathrm{F}_{(1,11)}=\right.$ $4.34 ; \mathrm{p}=0.061)$ and retrace $\left(\mathrm{F}_{(1,11)}=0.17 ; \mathrm{p}=0.686\right)$ percentages. However, the velocity on the first trial did significantly differ from the twelfth trial $\left(\mathrm{F}_{(1,11)}=\right.$ $6.72 ; p=0.025)$. As is visible in Figure 5, this was due to a slower performance on the first trial of the overlearned sequence compared to the previous sessions, probably related to the fact that subjects did not know in which order the conditions would be presented, and the possibility that subjects were initially more cautious compared to the situation in the dummy scanner (e.g., trying harder to prevent excessive head motion). In contrast, stronger changes in performance were expected for the sequence which was encountered in the first scanning session, but was not trained extensively afterwards ('OLD'). This expectation was confirmed for both the achieved velocity (increasing from 51 to $\left.65 \mathrm{~mm} / \mathrm{s} ; \mathrm{F}_{(1,11)}=12.26 ; \mathrm{p}=0.005\right)$ and the percentage of stops (decreasing from 39 to $\left.18 \% ; \mathrm{F}_{(1,11)}=40.89 ; \mathrm{p}=0.000\right)$. Finally, being confronted with a 
completely novel maze ('NEW') should also lead to changes in behavior across the presented repetitions. Such improvements in performance were indeed found for all behavioral measures: the velocity increased from 47 to $70 \mathrm{~mm} / \mathrm{s}$ $\left(F_{(1,11)}=61.63 ; p=0.000\right)$, while the stop and retrace percentages decreased from 32 to $16 \%\left(\mathrm{~F}_{(1,11)}=34.91 ; \mathrm{p}=0.000\right)$ and from 4 to $0 \%\left(\mathrm{~F}_{(1,11)}=5.34 ; \mathrm{p}=\right.$ 0.041 ), respectively.

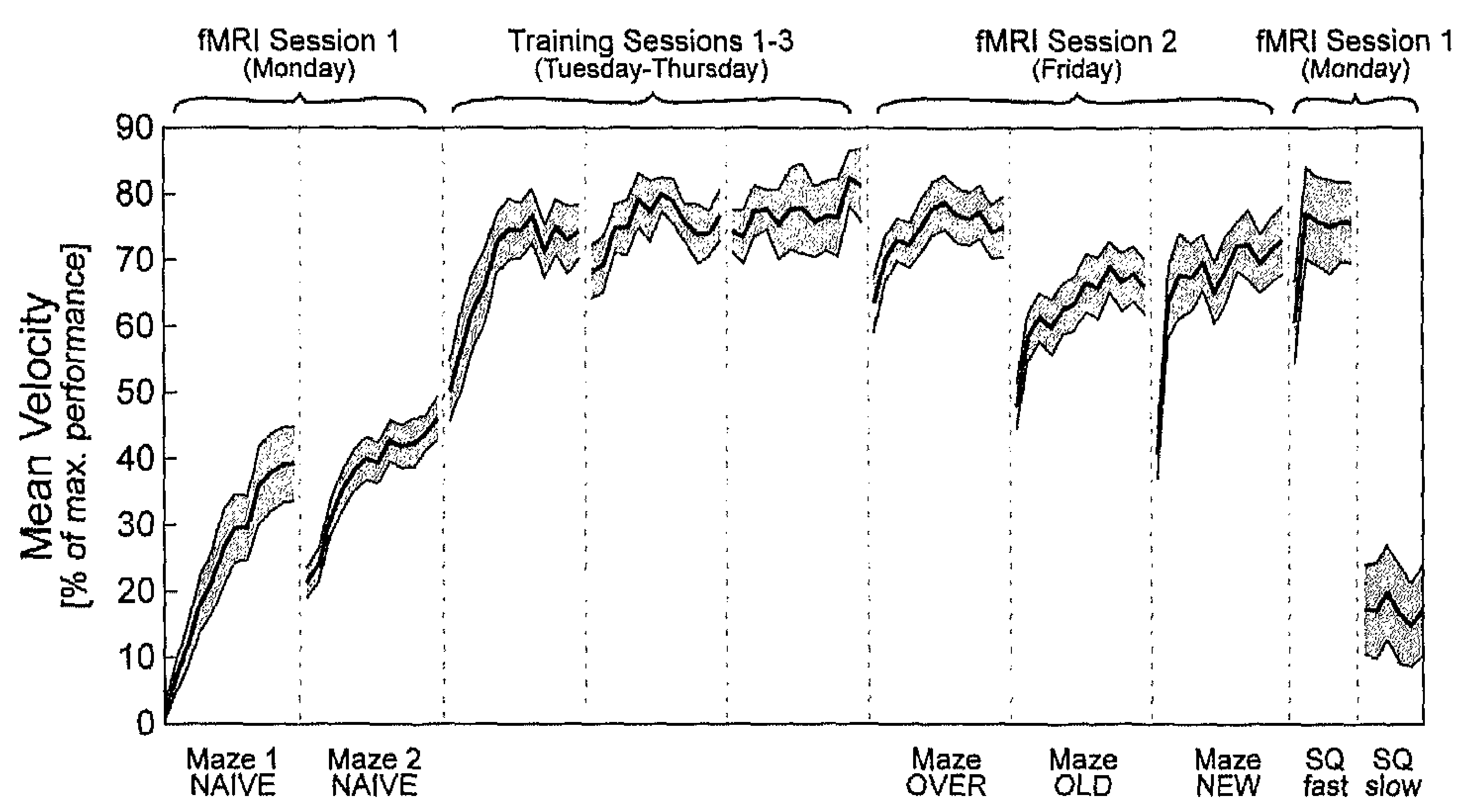

Figure 5. Behavioral results on the achieved velocity in scanning session 2 , embedded in an overview of the learning curve across all conditions. The curve reflects the min-max normalized velocity performance across subjects, individually normalized relative to the maximum performance achieved across all maze tracing trials. The shaded regions indicate the standard errors for the calculated trial means.

\subsubsection{Imaging Data}

A first observation regarding the imaging data of the second $\mathrm{AMRI}$ session was that a highly similar network of areas was active across the different tracing conditions, i.e. irrespective of the amount of preceding practice. In close correspondence to the general maze tracing network as identified in the first scanning session (Figure 3), separate contrasts for each condition against baseline resulted in remarkably overlapping activation patterns (Figure 6). This implies that the amount of practice does not dramatically affect the general constellation of the underlying neural architecture at the systems level. On the other hand, the presented contrasts nevertheless suggested that there might be some degree of differentiation related to the level of proficiency, as indicated by the apparent lack of right posterior parietal activation during the tracing of the overlearned sequence. 
a) Maze(NEW) $>$ Baseline

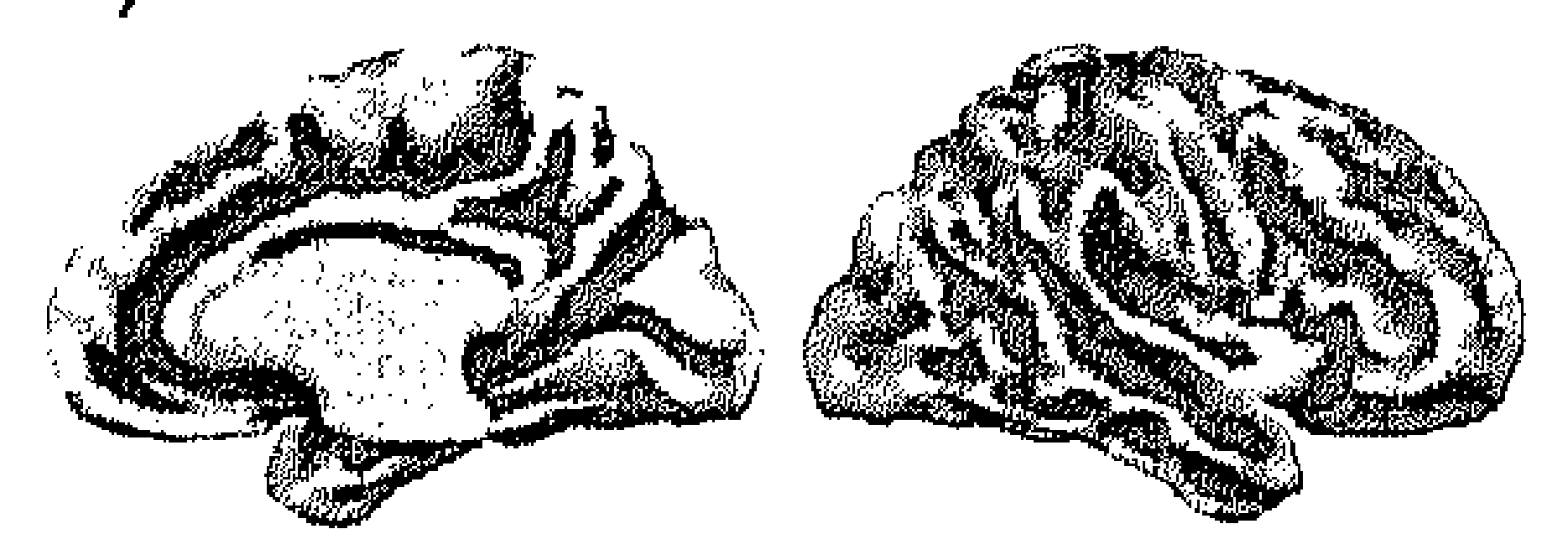

b) Maze (OLD) > Baseline

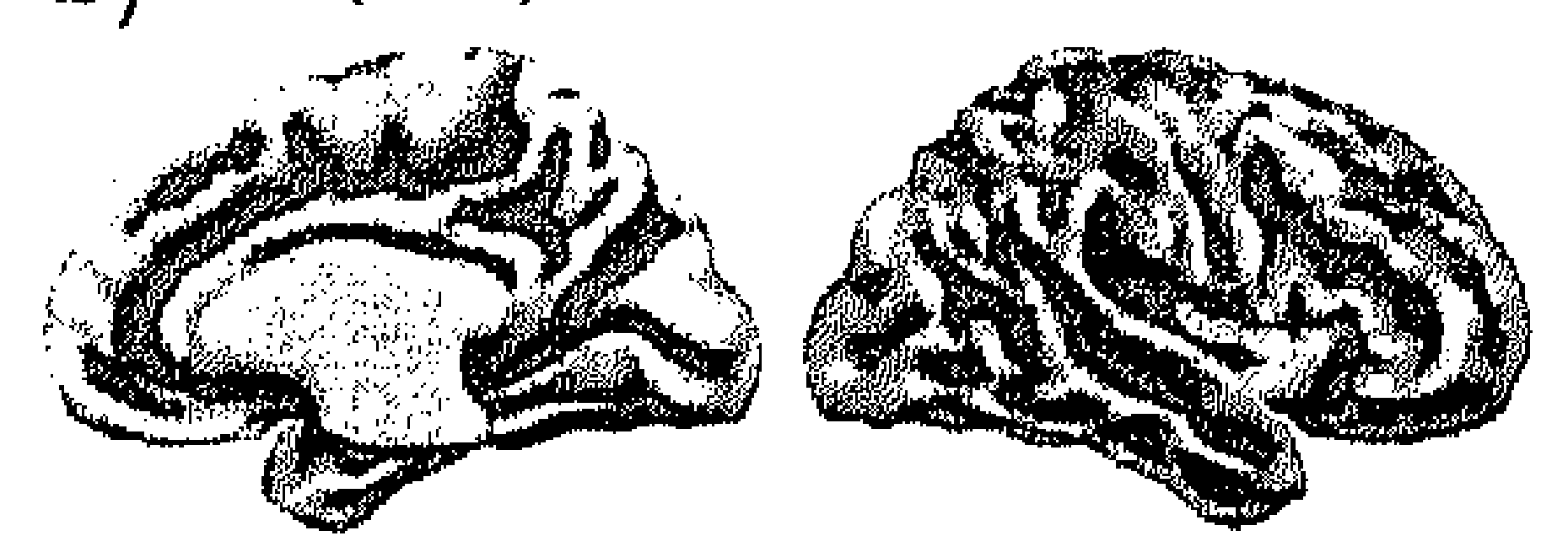

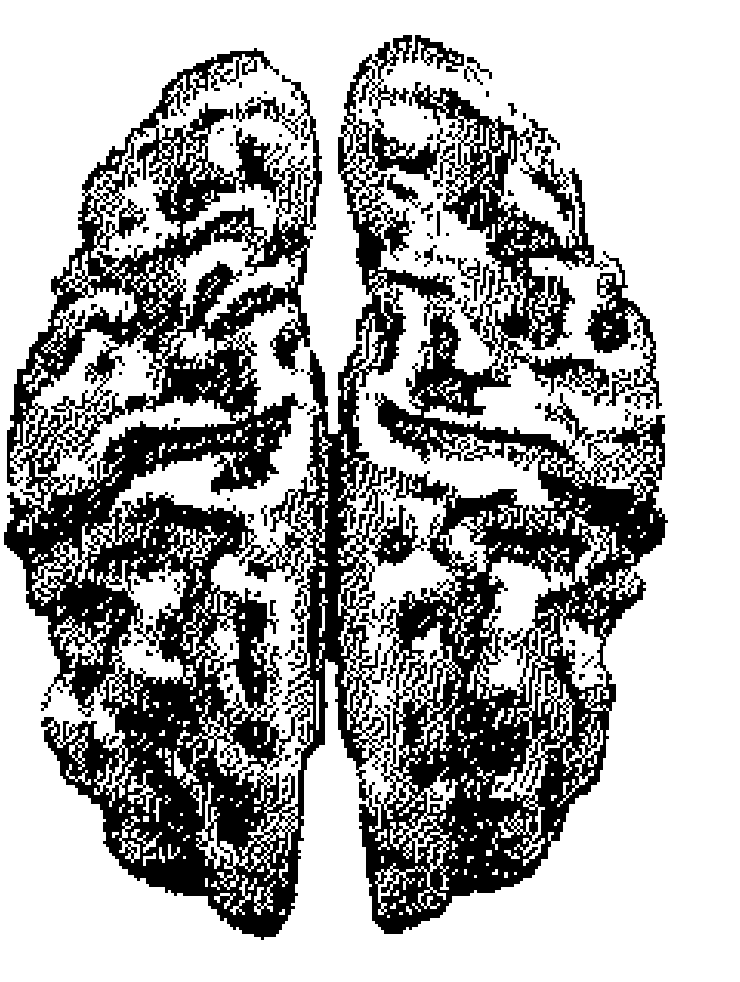
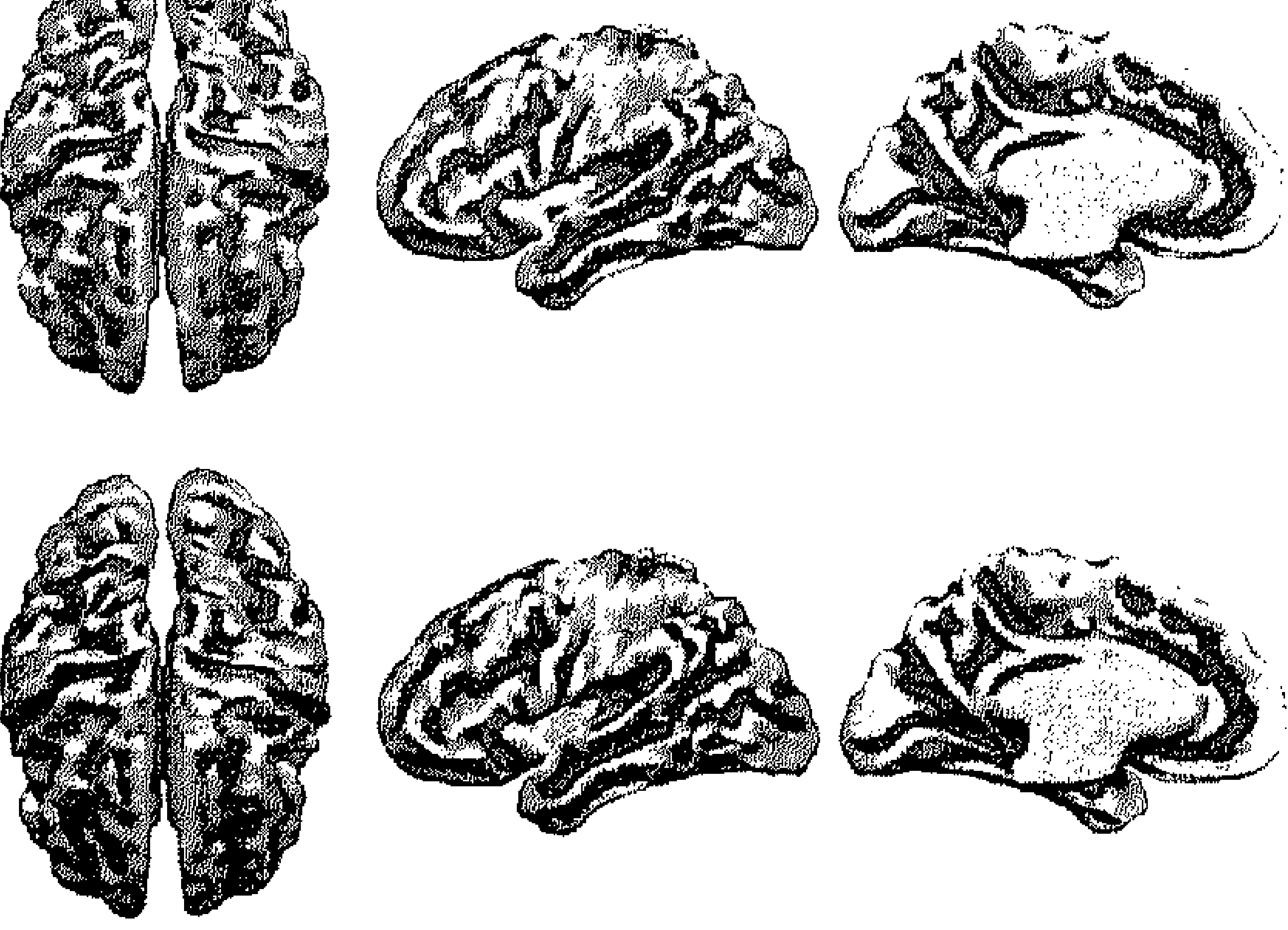

C) Maze(OVER) > Baseline
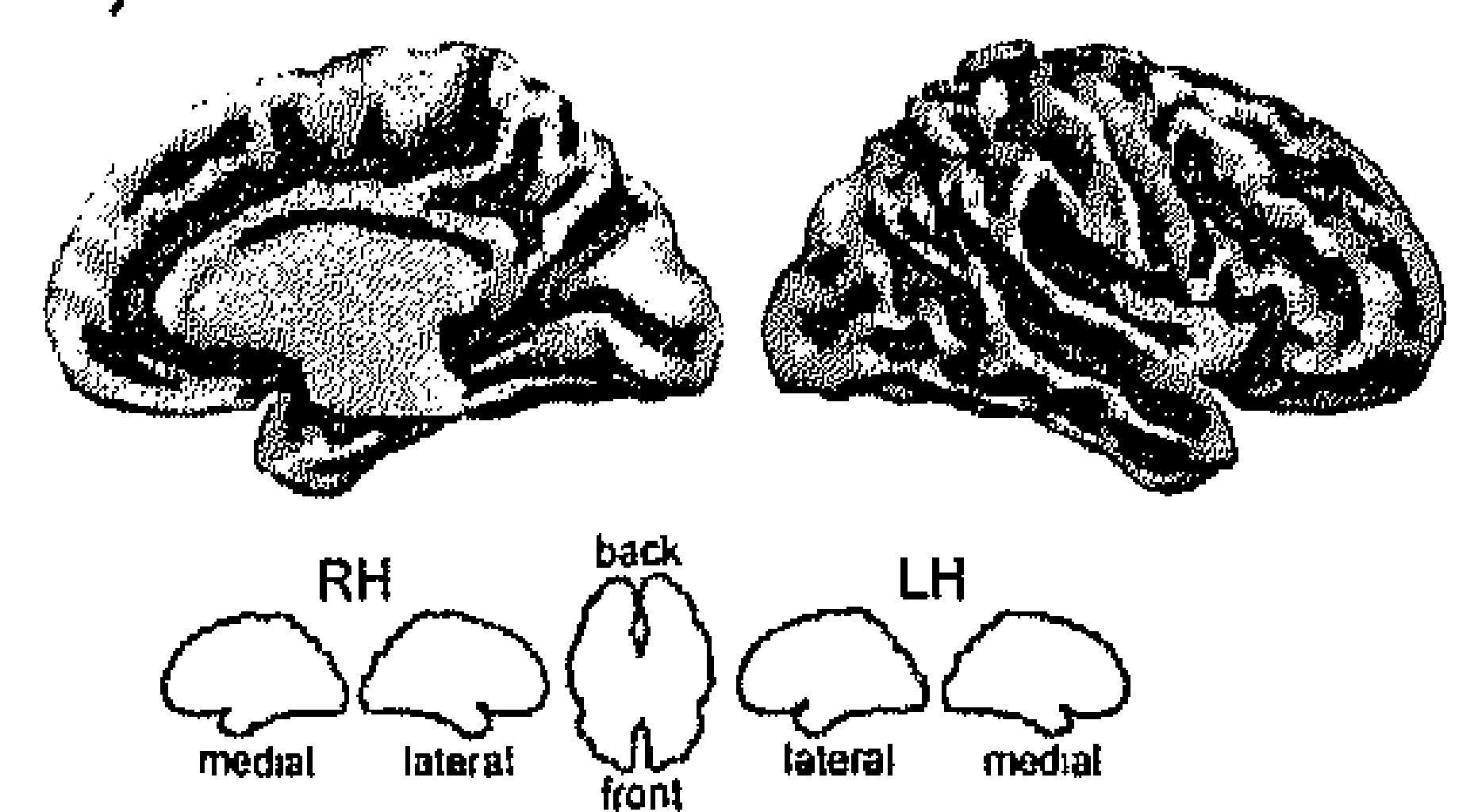
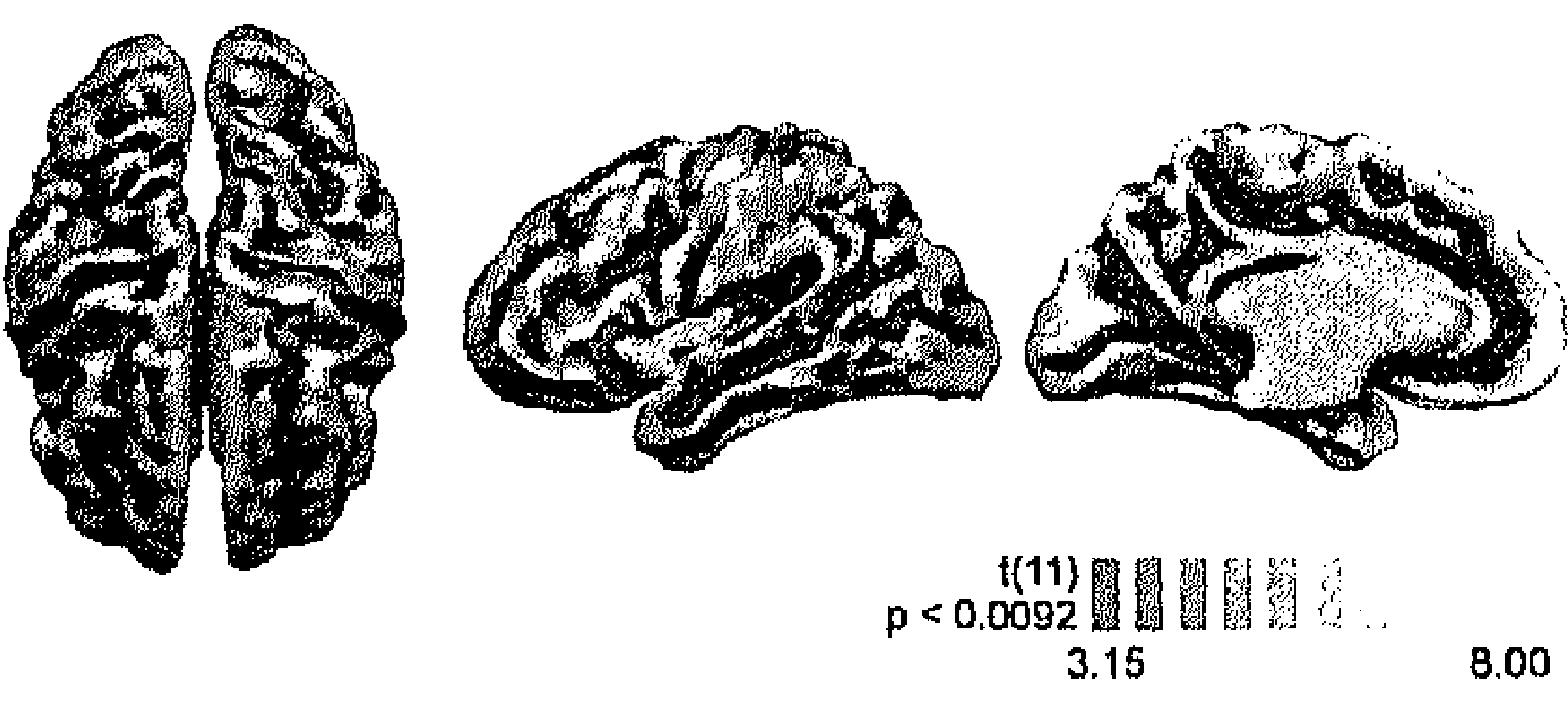

Figure 6. Group activation maps from session 2 for all three tracing conditions (pooled across 12 trials). a) Activation pattern found when contrasting the tracing of the completely novel maze with the resting baseline. b) Areas involved in the tracing of the maze which was encountered in session 1 but was not trained afterwards. c) Areas that respond while performing the overlearned movement sequence. Note how similar the identified networks are, and that the regions showing learning-specific responses in session 1 still seem to be involved in all conditions (except for the right posterior parietal area in the overlearned condition).

Abbreviations: $\mathrm{RH}$, right hemisphere; $\mathrm{LH}$, left hemisphere.

To assess the continuing involvement of areas that were previously designated to show learning-related activation changes, separate random effects contrasts were computed for the ROIs defined in the first session. However, due to the rapid changes in activation levels that can occur during learning (as was demonstrated above for scanning session 1), the effects of training were not examined by contrasting all trials of the different tracing conditions integrally. Instead, the behavioral data were again used to separate early from late stages of (within-session) learning. As shown in Table 2, all ROIs showed a significant decrease in activation during the tracing of the novel sequence as learning progressed. 


\begin{tabular}{|c|c|c|c|c|c|c|c|c|c|}
\hline \multirow{2}{*}{$\begin{array}{c}\text { Region } \\
\text { of } \\
\text { Interest }\end{array}$} & \multirow[t]{2}{*}{$\begin{array}{l}\text { Hemi- } \\
\text { sphere }\end{array}$} & \multicolumn{8}{|c|}{$\begin{array}{c}\text { Significance of Activation Changes } \\
\text { t-values and probabilitics for contrasts (RFX ROI-GLM; } n=12 \text { ) }\end{array}$} \\
\hline & & $\begin{array}{l}\text { NEW } \\
\text { Early }\end{array}$ & $>\underset{\text { Late }}{\text { NEW }}$ & $\begin{array}{l}\text { OLD } \\
\text { Early } \\
+\end{array}$ & $>\underset{\text { Late }}{\text { OLD }}$ & $\begin{array}{l}\text { OVER } \\
\text { Early }\end{array}$ & $>\underset{\text { Late }}{\text { OVER }}$ & $\begin{array}{c}\text { NEW } \\
\text { Early } \\
t\end{array}$ & $>>\begin{array}{c}\text { OVER } \\
\text { Early } \\
\text { (n) }\end{array}$ \\
\hline PMd & $\begin{array}{l}\mathrm{LH} \\
\mathrm{RH}\end{array}$ & $\begin{array}{l}3.73 \\
2.91\end{array}$ & $\begin{array}{l}(0.003)^{* *} \\
(0.014)^{*}\end{array}$ & $\begin{array}{l}2.54 \\
3.31\end{array}$ & $\begin{array}{l}(0.028)^{*} \\
(0.007)^{*}\end{array}$ & $\begin{array}{l}0.06 \\
0.76\end{array}$ & $\begin{array}{l}(0.951) \\
(0.463)\end{array}$ & $\begin{array}{l}2.21 \\
1.41\end{array}$ & $\begin{array}{l}(0.049)^{*} \\
(0.186)\end{array}$ \\
\hline PMv & $\begin{array}{l}\mathrm{LH} \\
\mathrm{RH}\end{array}$ & $\begin{array}{l}5.22 \\
5.81\end{array}$ & $(0.000)^{* *}$ & $\begin{array}{l}3.52 \\
3.56\end{array}$ & $\begin{array}{l}(0.005)^{*} \\
(0.004)^{* *}\end{array}$ & $\begin{array}{l}0.21 \\
1.88\end{array}$ & $\begin{array}{l}(0.842) \\
(0.088)\end{array}$ & $\begin{array}{l}2.39 \\
1.92\end{array}$ & $\begin{array}{l}(0.036)^{*} \\
(0.081)\end{array}$ \\
\hline InfPCS & $\begin{array}{l}\mathrm{LH} \\
\mathrm{RH}\end{array}$ & $\begin{array}{l}6.17 \\
5.90\end{array}$ & $\begin{array}{l}(0.000)^{* *} \\
(0.000)^{* *}\end{array}$ & $\begin{array}{l}3.03 \\
3.01\end{array}$ & $\begin{array}{l}(0.012)^{*} \\
(0.012)^{*}\end{array}$ & $\begin{array}{l}2.51 \\
2.04\end{array}$ & $\begin{array}{l}(0.029)^{*} \\
(0.067)\end{array}$ & $\begin{array}{l}3.20 \\
2.32\end{array}$ & $\begin{array}{l}(0.008)^{*} \\
(0.041)^{*}\end{array}$ \\
\hline alPS & $\begin{array}{l}\mathrm{LH} \\
\mathrm{RH}\end{array}$ & $\begin{array}{l}5.85 \\
6.49\end{array}$ & $\begin{array}{l}(0.000)^{* *} \\
(0.000)^{* *}\end{array}$ & $\begin{array}{l}3.09 \\
3.13\end{array}$ & $\begin{array}{l}(0.010)^{*} \\
(0.010)^{*}\end{array}$ & $\begin{array}{l}1.51 \\
1.91\end{array}$ & $\begin{array}{l}(0.160) \\
(0.083)\end{array}$ & $\begin{array}{l}2.86 \\
2.42\end{array}$ & $\begin{array}{l}(0.016)^{*} \\
(0.034)^{*}\end{array}$ \\
\hline mIPS & $\begin{array}{l}\mathrm{LH} \\
\mathrm{RH}\end{array}$ & 4.04 & $(0.002)^{* *}$ & 2.70 & $(0.021)^{*}$ & 0.31 & $-(0.760)$ & 2.81 & $(0.017)^{*}$ \\
\hline SPL & $\begin{array}{l}\text { LH } \\
\text { RH }\end{array}$ & $\begin{array}{l}3.89 \\
5.29\end{array}$ & $\begin{array}{l}(0.003)^{* *} \\
(0.000)^{* *}\end{array}$ & $\begin{array}{l}2.96 \\
2.70\end{array}$ & $\begin{array}{l}(0.013)^{*} \\
(0.021)^{*}\end{array}$ & $\begin{array}{r}-1.56 \\
0.16\end{array}$ & $\begin{array}{l}(0.146) \\
(0.876)\end{array}$ & $\begin{array}{l}5.30 \\
3.94\end{array}$ & $\begin{array}{l}(0.000)^{* *} \\
(0.002)^{* *}\end{array}$ \\
\hline
\end{tabular}

Table 2. ROI-GLM results for scanning session 2 . The table shows that learning-related decreases occurred for both the 'NEW' and the previously encountered ('OLD') sequence in all implicated regions, whereas no additional decreases were observed for the extensively trained sequence ('OVER'; except in the left InfPCS). Directly comparing the early stages of the novel and overlearned sequence conditions revealed a significantly higher activation for the novel sequence in almost all ROIs.

${ }^{*} \mathrm{p}<0.05$ (uncorrected); ${ }^{* *} \mathrm{p}<0.0045$ (Bonferroni-corrected for the number of included ROIs). Abbreviations as in Table 1.

As depicted in the event-related average plots in Figure 7 (first column), ensuing practice of the novel sequence led to a drop in activation to the level which was associated with the execution of a well-learned action pattern. Moreover, a similar learning-related decrease was observed throughout the entire network for the sequence which was previously encountered in session 1, but was not practiced any further afterwards (second column of Figure 7; Table 2). As expected if this previous experience would still have an effect, the overall difference between the early and late stage of learning was less pronounced compared to the completely novel case. Importantly, the learning-related ROIs generally did not show a significant change in activation level for the overlearned sequence, presumably because no additional learning took place (column three in Figure 7). Apparently, the completely novel sequence was learned relatively fast, a finding which both neural and behavioral probes of learning converged on. Consequently, the strongest divergence in activation 
levels between the novel and overlearned sequence would be expected in the early stages of task execution. The final column in Table 2 shows that a large majority of ROIs (80\%) indeed responded more strongly during the initial stage of learning the new movement sequence than during the first trials of executing the overlearned sequence. Similar to what was found for the second maze in the first scanning session, additional 'whole brain'-contrasts revealed that the amplitude of the bilateral SMA response (Talairach coordinates: $-5,-11,56$ [left]; 7, -7, 54 [right]) decreased when comparing early versus late performance on both the 'NEW' and 'OLD' sequence. No further decrease was observed for the overlearned movement pattern, consistent with the general response profile of the other areas as described above. The only region which showed an increased activation level during the early phase of executing the overlearned compared to the completely novel sequence was located in the left posterior putamen (Talairach coordinates: $-27,-9,-8$ ).

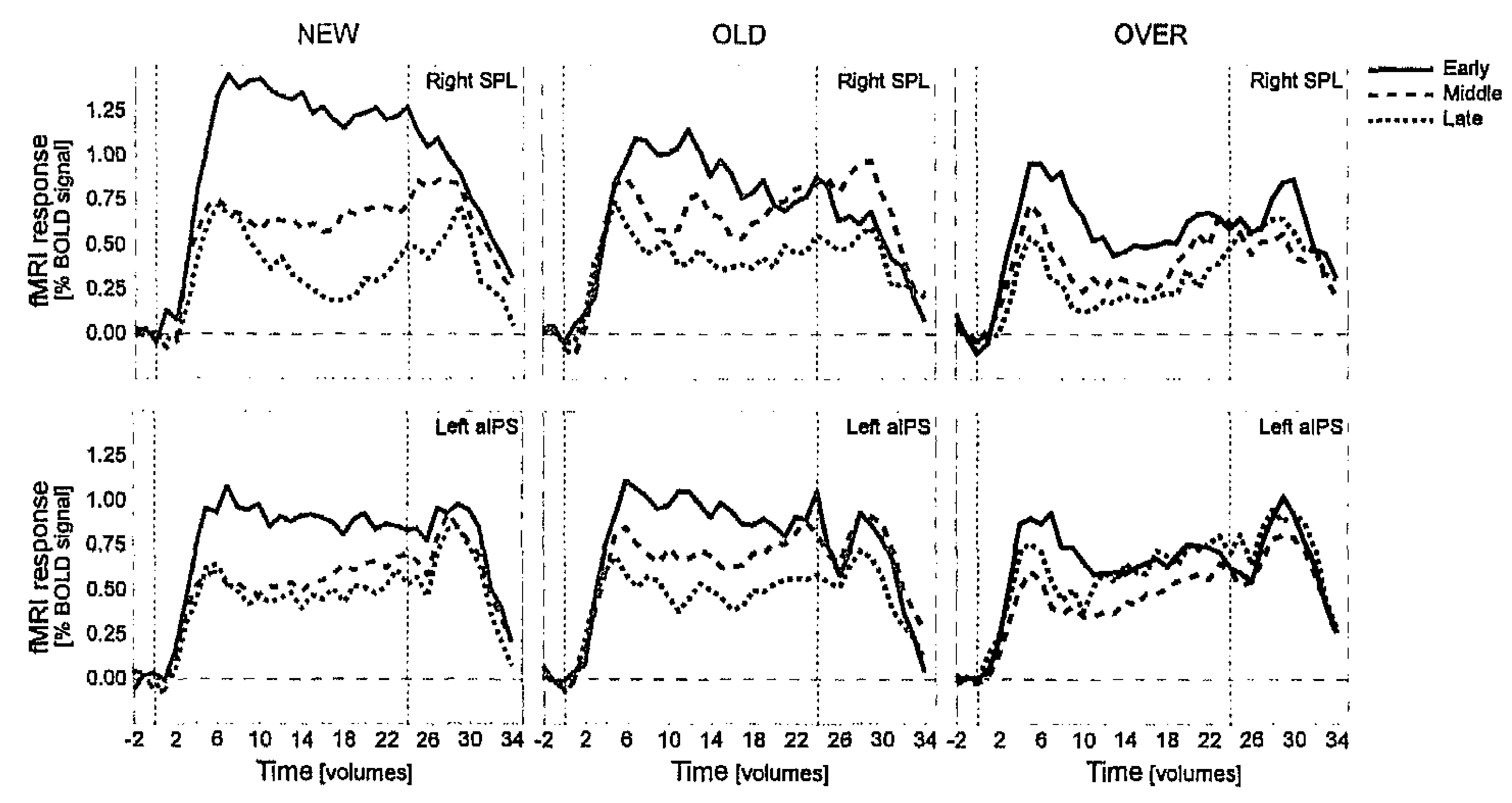

Figure 7. Event-related responses to the different tracing conditions in scanning session 2 (averaged across subjects). The top and bottom rows show the activation pattern for the right SPL and left aIPS ROIs, respectively. The columns represent the three tracing conditions, and the separate lines reflect the different intra-session performance stages. Note that the activation level decreases for both the 'NEW' and 'OLD' sequence, whereas the response's amplitude for the overlearned sequence ('OVER') is already weaker at the early stage and does not significantly decrease any further across the remaining trials. 
Motor sequence learning

\section{Discussion}

The current study aimed to provide insights into the neural dynamics associated with the nonvisual motor learning of 2-dimensional continuous movement sequences. Learning-related changes in brain activity were examined on two different time scales: both within the time frame of a single scanning session and across a time window of several days involving multiple training sessions.

\subsection{Identified learning-related activations}

The presented data show that the execution of the required pen tracing movements was accompanied by activations in a distributed network of (sub)cortical regions, replicating earlier PET results (van Mier et al., 1998; van Mier et al., 2004). Within this network, only the contralateral M1/S1 and ipsilateral cerebellar foci displayed clear velocity-dependent responses, in accordance with previous studies (e.g., Turner et al., 1998; Riecker et al., 2003). In contrast, several regions were more strongly engaged during the execution and learning of the new movement sequences. Since their activation level was significantly higher during the tracing of the mazes compared to either of the control conditions, and these regions did not differentiate between the fast and slow square tracing conditions per se, the observed responses truly reflected the ongoing learning process and were free from confounds related to concomitant increases in movement velocity. The regions involved in learning were generally activated bilaterally, showing a high degree of symmetry across both hemispheres. Frontal learning-related activity was restricted to the dorsal and ventral premotor cortices, known to be involved in the preparation and execution of voluntary actions (Wise et al., 1997; Rizzolatti and Luppino, 2001), as well as the production and learning of specific movement sequences (e.g., Jueptner et al., 1997; Harrington et al., 2000). The fact that learningrelated activations in the current study were defined by comparing the maze learning with the tracing of the geometrically less complex squares might seem problematic in the light of reported PMd modulations related to sequence complexity (Haaland et al., 2004). However, the identified changes in activation patterns due to practice suggest this region is not driven by the complexity of the required movements per se. Another concern that might be raised with respect to the observed PMd activation is the vicinity of the frontal eye fields (FEF), which could suggest that the learning-related effects are confounded by non-specific changes in eye movement patterns, even though the task was performed while keeping the eyes closed. Although there has been considerable debate on the exact location of the FEF in humans (Tehovnik et al., 2000), Amiez and colleagues (2006) have recently demonstrated a clear distinction between the PMd and FEF based on intra-subject comparisons using fMRI. Taking their distinct localization as a guideline, and noting that a previous PET 
study using the same maze tracing task did not find any task-related changes in recorded eye movement patterns (van Mier et al., 1998), it is most likely that the activations reported here truly reflect PMd activity.

The remaining learning-related activations were all located in the parietal lobe, comprising areas which constitute the main sources of input to the premotor areas described above (Rizzolatti and Luppino, 2001). The most laterally located activation site was found in the bilateral inferior postcentral sulcus, which in humans has recently been classified as the most rostral part of the inferior parietal lobule based on cytoarchitectonic data (Caspers et al., 2006) and which is primarily connected to nearby somatosensory and parietal areas as shown in monkeys (Rozzi et al., 2006). Another lateral region was identified bilaterally in the anterior IPS, corresponding to the human homologue of the macaque AIP (anterior intra-parietal) sulcus (Culham and Valyear, 2006), which has been shown to be involved in visually-guided grasping (Castiello, 2005) and visuo-tactile integration (Grefkes et al., 2002). The more posterior activation in the left medial IPS/middle SPL constitutes another putative human homologue to a well-known area in the monkey, namely medial intraparietal area MIP (Grefkes and Fink, 2005). Medial IPS activity was previously reported in humans during goal-directed joystick movements towards visually presented targets, even in the absence of direct visual feedback (Grefkes et al., 2004). The final activation site showing learning-related responses in the current study was located in the posterior part of the SPL, adjacent to the medial IPS activity. Together these regions form the so-called parietal reach region (PRR) in the macaque, which has been associated with the control of reaching movements (e.g., Snyder et al., 1998). This region is particularly interesting because it is believed to code what has been termed 'motor intentions', relatively abstract movement plans specifying both the goal of a movement and the type of movement required to achieve this goal (Andersen and Buneo, 2002; Quian Quiroga et al., 2006). However, similar posterior parietal activations have also been reported in the context of (both spatial and non-spatial) attention switches (Behrmann et al., 2004). Even though a detailed functional description of the identified areas can not be derived based on the current experiment, and interspecies homologies need to be generally stated with caution (Culham et al., 2006), the message conveyed by the foregoing discussion is that areas which have been predominantly implicated in the organization of sequential behavior and visuomotor transformations in the context of goal-directed movements, are shown here to be additionally involved in the learning of novel continuous movement sequences based on somatosensory and proprioceptive input alone.

Interestingly, no tracing- or learning-related activations were found in the dorso-lateral prefrontal cortex (DLPFC). Recruitment of the DLPFC might have been expected based on current models on motor sequence learning (Ashe et al., 2006) and reports on sequence-specific activation patterns identified using 
single-cell recordings (Averbeck et al., 2002, 2006). However, by varying the correspondence between behavioral goals and the movements required to achieve them, Mushiake and colleagues (2006) recently showed that the DLPFC does not necessarily code specific movement sequences, but rather their more abstract consequences in terms of behaviorally relevant subgoals (see also Saito et al., 2005). The lack of DLPFC involvement in the present experiment suggests that the task requirements were met at the motor system level, underlining the paradigm's focus on motor aspects of learning instead of its more cognitive and strategy-dependent characteristics.

\subsection{Brain activation changes over the course of learning}

Already within the first scanning session, the contributions of the areas listed above started to change while learning progressed. Although still being moderate initially, more pronounced learning-related decreases in activation were observed later on as performance improved. Interestingly, these widespread experience-dependent decreases in brain activation were replicated four days later, in the second scanning session. Here, decreases were found both when a completely novel sequence was presented, and when learning continued on the maze which was traced previously in session 1 but was not extensively trained afterwards. Conversely, no such additional intra-session learning was found for the overlearned sequence. These findings stress the specificity of the observed learning-related activations, and provide a clear illustration of practice-induced changes in neural processing. However, the obtained results additionally showed that even though the activation levels of the identified regions markedly decreased with practice, there was a substantial overlap in activation patterns across sessions and levels of proficiency. This consistency indicates that a similar network of regions is engaged throughout the course of skill acquisition, although the required processing is performed more efficiently as learning evolves. Such an interpretation is in line with recent results from studies investigating extended motor learning across multiple scanning sessions (de Weerd et al., 2003; Wu et al., 2004; Poldrack et al., 2005), as well as studies contrasting the neural correlates of learning in novices versus experts (Haslinger et al., 2004; Meister et al., 2005).

On the other hand, models on motor sequence learning have proposed dynamic shifts in activation patterns, leading to increased involvement of subcortical structures in later learning stages (Doyon \& Benali, 2005; Ashe et al., 2006). Although the subcortical activations identified in the first session of the current study were neither specifically involved in learning nor restricted to a particular time window, differential responses were found for the posterior putamen in the second scanning session. This structure was more strongly engaged during the early phase of tracing the overlearned compared to the novel maze, pointing to a function in the re-instatement of a previously learned movement set (Miyachi et 


\section{Chapter 3}

al., 2002; Lehericy et al., 2005). Nevertheless, the response profile in the posterior putamen does not warrant a description of the whole learning process in terms of a reorganization of functional activations (as defined following the classification scheme proposed by Kelly and Garavan, 2005), because the same structure was involved when tracing the novel maze (albeit to a lesser degree). Moreover, although practice-induced activation decreases could be observed throughout the cortical network of learning-related regions, the general layout of this network remained unchanged across different levels of expertise.

\section{Conclusion}

In the presented study, the acquisition of novel continuous movement patterns was examined across two different time windows. Using detailed behavioral recordings to discriminate between early and late stages of learning at the single subject level, and the application of advanced inter-subject alignment procedures, changes in brain activity specific to the learning of the new movement sequences could be identified in a distributed network of cortical regions. Most importantly, learning-related decreases in activity were already observed within the course of a single scanning session, and were replicated several days later in a second experiment. Furthermore, the same bilateral network of learning-related areas was implicated during task execution after extensive practice across several days, although its contribution became less pronounced. This indicates that learning in the current continuous sequence task mainly results in more efficient processing in the recruited regions and does not cause a substantial reorganization of functional activations. 


\section{References}

Alkadhi, H., Crelier, G.R., Boendermaker, S.H., Golay, X., Hepp-Reymond, M-C., \& Kollias, S.S. (2002). Reproducibility of primary motor cortex somatotopy under controlled conditions. American Journal of Neuroradiology, 23, 1524-1532.

Amiez, C., Kostopoulos, P., Champod, A-S., \& Petrides, M. (2006). Local morphology predicts functional organization of the dorsal premotor region in the human brain. Journal of Neuroscience, 26, 2724-2731.

Andersen, R.A., \& Buneo, C.A. (2002). Intentional maps in posterior parietal cortex. Annual Review of Neuroscience, 25, 189-220.

Ashe, J., Lungu, O.V., Basford, A.T., \& Lu, X. (2006). Cortical control of motor sequences. Current Opinion in Neurobiology, 16, 213-221.

Averbeck, B.B., Chafee, M.V., Crowe, D.A., \& Georgopoulos, A.P. (2002). Parallel processing of serial movements in prefrontal cortex. Proceedings of the National Academy of Sciences USA, 99, 13172-13177.

Averbeck, B.B., Sohn, J.W., \& Lee, D. (2006). Activity in prefrontal cortex during dynamic selection of action sequences. Nature Neuroscience, 9, 276-282.

Behrmann, M., Geng, J.J., \& Shomstein, S. (2004). Parietal cortex and attention. Current Opinion in Neurobiology, 14, 212-217.

Bischoff-Grethe, A., Goedert, K.M., Willingham, D.T., \& Grafton, S.T. (2004). Neural substrates of response-based sequence learning using AMRI. Journal of Cognitive Neuroscience, 16, 127-138.

Caspers, S., Geyer, S., Schleicher, A., Mohlberg, H., Amunts, K., \& Zilles, K. (2006). The human inferior parietal cortex: Cytoarchitectonic parcellation and interindividual variability. NeuroImage, 33, 430-448.

Castiello, U. (2005). The neuroscience of grasping. Nature Reviews Neuroscience, 6, 726-736.

Clegg, B.A., DiGirolamo, G.J., \& Keele, S.W. (1998). Sequence learning. Trends in Cognitive Sciences, 2, 275-281.

Culham, J.C., \& Valyear, K.F. (2006). Human parietal cortex in action. Current Opinion in Neurobiology, 16, 205-212.

Culham, J.C., Cavina-Pratesi, C., \& Singhal, A. (2006) The role of parietal cortex in visuomotor control: What have we learned from neuroimaging? Neuropsychologia, 44, 2668-2684. 
Chapter 3

Deichmann, R., Schwarzbauer, C., \& Turner, R. (2004). Optimisation of the 3D MDEFT sequence for anatomical brain imaging: technical implications at 1.5 and $3 \mathrm{~T}$. NeuroImage, 21, 757-767.

de Weerd, P., Reinke, K., Ryan, L., McIsaac, T., Perschler, P., Schnyer, D., Trouard, T., \& Gmitro, A. (2003). Cortical mechanisms for acquisition and performance of bimanual motor sequences. NeuroImage, 19, 1405-1416.

Doyon, J., \& Ungerleider, L.G. (2002). Functional anatomy of motor skill learning. In L.R. Squire, \& D.L. Schacter (Eds.), Neuropsychology of memory (pp. 225-238). New York (NY): Guilford Press.

Doyon, J., \& Benali, H. (2005). Reorganization and plasticity in the adult brain during learning of motor skills. Current Opinion in Neurobiology, 15, 161-167.

Floyer-Lea, A., \& Matthews, P.M. (2004). Changing brain networks for visuomotor control with increased movement automaticity. Journal of Neurophysiology, 92, 24052412.

Goebel, R., Esposito, F., \& Formisano, E. (2006). Analysis of functional image analysis contest (FIAC) data with BrainVoyager QX: From single-subject to cortically aligned group General Linear Model analysis and self-organizing group Independent Component Analysis. Human Brain Mapping, 27, 392-401.

Grefkes, C., Weiss, P.H., Zilles, K., \& Fink, G.R. (2002). Crossmodal processing of object features in human anterior intraparietal cortex: an fMRI study implies equivalencies between humans and monkeys. Neuron, 35, 173-184.

Grefkes, C., Ritzl, A., Zilles, K., \& Fink, G.R. (2004). Human medial intraparietal cortex subserves visuomotor coordinate transformation. NeuroImage, 23, 1494-1506.

Grefkes, C., \& Fink, G.R. (2005). The functional organization of the intraparietal sulcus in humans and monkeys. Journal of Anatomy, 207, 3-17.

Haaland, K.Y., Elsinger, C.L., Mayer, A.R., Durgerian, S., \& Rao, S.M. (2004). Motor sequence complexity and performing hand produce differential patterns of hemispheric lateralization. Journal of Cognitive Neuroscience, 16, 621-636.

Harrington, D.L., Rao, S.M., Haaland, K.Y., Bobholz, J.A., Mayer, A.R., Binder, J.R., \& Cox, R.W. (2000). Specialized neural systems underlying representations of sequential movements. Journal of Cognitive Neuroscience, 12, 56-77.

Haslinger, B., Erhard, P., Altenmueller, E., Hennenlotter, A., Schwaiger, M., Graefin von Einsiedel, H., Rummeny, E., Conrad, B., \& Ceballos-Baumann, A. (2004). Reduced recruitment of motor association areas during bimanual coordination in concert pianists. Human Brain Mapping, 22, 206-215. 
Jueptner, M., Stephan, K.M., Frith, C.D., Brooks, D.J., Frackowiak, R.S.J., \& Passingham, R.E. (1997). Anatomy of motor learning. I. Frontal cortex and attention to action. Journal of Neurophysiology, 77, 1313-1324.

Keele, S.W., Ivry, R., Mayr, U., Hazeltine, E., \& Heuer, H. (2003). The cognitive and neural architecture of sequence representation. Psychological Review, 110, 316-339.

Kelly, A.M.C., \& Garavan, H. (2005) Human functional neuroimaging of brain changes associated with practice. Cerebral Cortex, 15, 1089-1102.

Kriegeskorte, N., \& Goebel, R. (2001). An efficient algorithm for topologically correct segmentation of the cortical sheet in anatomical MR volumes. NeuroImage, 14, 329346.

Lehericy, S., Benali, H., van de Moortele, P-F., Pelegrini-Issac, M., Waechter, T., Ugurbil, K., \& Doyon, J. (2005). Distinct basal ganglia territories are engaged in early and advanced motor sequence learning. Proceedings of the National Academy of Sciences USA, 102, 12566-12571.

Lotze, M., Erb, M., Flor, H., Huelsmann, E., Godde, B., \& Grodd, W. (2000). fMRI evaluation of somatotopic representation in human primary cortex. NeuroImage, 11, $473-481$.

Meister, I., Krings, T., Foltys, H., Boroojerdi, B., Mueller, M., Toepper, R., \& Thron, A. (2005). Effects of long-term practice and task complexity in musicians and nonmusicians performing simple and complex motor tasks: implications for cortical motor organization. Human Brain Mapping, 25, 345-352.

Miyachi, S., Hikosaka, O., \& Lu, X. (2002). Differential activation of monkey striatal neurons in the early and late stages of procedural learning. Experimental Brain Research, 146, 122-126.

Mushiake, H., Saito, N., Sakamoto, K., Itoyama, Y., \& Tanji, J. (2006). Activity in the lateral prefrontal cortex reflects multiple steps of future events in action plans. Neuron, $50,631-641$.

Nissen, M.J., \& Bullemer, P. (1987). Attentional requirements of learning: Evidence from performance measures. Cognitive Psychology, 19, 1-32.

Oldfield, R.C. (1971). The assessment and analysis of handedness: the Edinburgh Inventory. Neuropsychologia, 9, 97-113.

Poldrack, R.A. (2000). Imaging brain plasticity: conceptual and methodological issues a theoretical review. NeuroImage, 12, 1-13. 
Chapter 3

Poldrack, R.A., Sabb, F.W., Foerde, K., Tom, S.M., Asarnow, R.F., Bookheimer, S.Y., \& Knowlton, B.J. (2005). The neural correlates of motor skill automaticity. Journal of Neuroscience, 25, 5356-5364.

Quian Quiroga, R., Snyder, L.H., Batista, A.P., Cui, H., \& Andersen, R.A. (2006). Movement intention is better predicted than attention in the posterior parietal cortex. Journal of Neuroscience, 26, 3615-3620.

Reithler, J., Reithler, H., van den Boogert, E., Goebel, R., \& van Mier, H. (2006). Resistance-based high resolution recording of predefined 2-dimensional pen trajectories in an fMRI setting. Journal of Neuroscience Methods, 152, 10-17.

Riecker, A., Wildgruber, D., Mathiak, K., Grodd, W., \& Ackermann, H. (2003). Parametric analysis of rate-dependent hemodynamic response functions of cortical and subcortical brain structures during auditorily cued finger tapping: a fMRI study. NeuroImage, 18, 731-739.

Rizzolatti, G., \& Luppino, G. (2001). The cortical motor system. Neuron, 31, 889-901.

Rozzi, S., Calzavara, R., Belmalih, A., Borra, E., Gregoriou, G.G., Matelli, M., \& Luppino, G. (2006). Cortical connections of the inferior parietal cortical convexity of the macaque monkey. Cerebral Cortex, 16, 1389-1417.

Saito, N., Mushiake, H., Sakamoto, K., Itoyama, Y., \& Tanji, J. (2005). Representation of immediate and final behavioral goals in the monkey prefrontal cortex during an instructed delay period. Cerebral Cortex, 15, 1535-1546.

Sanes, J.N. (2003). Neocortical mechanisms in motor learning. Current Opinion in Neurobiology, 13, 225-231.

Snyder, L.H., Batista, A.P., \& Andersen, R.A. (1998). Change in motor plan, without a change in the spatial locus of attention, modulates activity in posterior parietal cortex. Journal of Neurophysiology, 79, 2814-2819.

Talairach, J., \& Tournoux, P. (1988). Co-planar stereotaxic atlas of the human brain. Stuttgart: G. Thieme.

Tanji, J. (2001). Sequential organization of multiple movements: involvement of cortical motor areas. Annual Review of Neuroscience, 24, 631-651.

Tehovnik, E.J., Sommer, M.A., Chou, I.H., Slocum, W.M., \& Schiller, P.H. (2000). Eye fields in the frontal lobes of primates. Brain Research Reviews, 32, 413-448.

Toni, I., Krams, M., Turner, R., \& Passingham, R.E. (1998). The time course of changes during motor sequence learning: a whole-brain fMRI study. Neurolmage, 8, 50-61. 
Turner, R.S., Grafton, S.T., Votaw, J.R., Delong, M.R., \& Hoffman, J.M. (1998). Motor subcircuits mediating the control of movement velocity: a PET study. Journal of Neurophysiology, 80, 2162-2176.

van Mier, H., Hulstijn, W., \& Petersen, S.E. (1993). Changes in motor planning during the acquisition of movement patterns in a continuous task. Acta Psychologica, 82, 291312.

van Mier, H., Tempel, L.W., Perlmutter, J.S., Raichle, M.E., \& Petersen, S.E. (1998). Changes in brain activity during motor learning measured with PET: effects of hand of performance and practice. Journal of Neurophysiology, 80, 2177-2199.

van Mier, H.I., Perlmutter, J.S., \& Petersen, S.E. (2004). Functional changes in brain activity during acquisition and practice of movement sequences. Motor Control, 8, 500520 .

Vaughan, J.T., Garwood, M., Collins, C.M., Liu, W., DelaBarre, L., Adrainy, G., Andersen, P., Merkle, H., Goebel, R., Smith, M.B., \& Ugurbil, K. (2001). 7T vs. 4T: RF power, homogeneity, and signal-to-noise comparison in head images. Magnetic Resonance in Medicine, 46, 24-30.

Wise, S.P., Boussaoud, D., Johnson, P.B., \& Caminiti, R. (1997). Premotor and parietal cortex: corticocortical connectivity and combinatorial computations. Annual Review of Neuroscience, 20, 25-42.

Wu, T., Kansaku, K., \& Hallett, M. (2004). How self-initiated memorized movements become automatic: a functional MRI study. Journal of Neurophysiology, 91, 1690-1698. 


\section{Chapter 4}

\section{Nonvisual motor learning influences abstract action observation}

Based on: Reithler, J., van Mier, H., Peters, J.C., \& Goebel, R. (2007).

Nonvisual motor learning influences abstract action observation. Current Biology, 17, 1201-1207. 
Chapter 4

\begin{abstract}
Neuroimaging studies have recently provided support for the existence of a human equivalent of the 'mirror neuron' system as first described in monkeys [1], involved in both the execution of movements, as well as the observation and imitation of actions performed by others (e.g., [2-6]). A widely held conception concerning this system is that the understanding of observed actions is mediated by a covert simulation process [7]. In the present AMRI experiment, this simulation process was probed by asking subjects to discriminate between visually presented trajectories which either did or did not match previously performed, yet unseen continuous movement sequences. A specific network of learning-related premotor and parietal areas was found to be reactivated when participants were confronted with their movements' visual counterpart. Moreover, the strength of these reactivations was dependent on the observers' experience with executing the corresponding movement sequence. These findings provide further support to the emerging view that embodied simulations during action observation engage widespread activations in cortical motor regions beyond the classically defined mirror neuron system. Furthermore, the obtained results extend previous work by showing experience-dependent perceptual modulations at the neural systems level based on nonvisual motor learning.
\end{abstract}




\section{Results}

In the current event-related functional Magnetic Resonance Imaging (IMRI) experiment, subjects were confronted with motion stimuli which constituted visual representations of the resulting paths of previously trained, yet unseen movement sequences. The presented study had two main goals. First, we wanted to investigate whether the actions implied by the observed dynamically drawn paths would be 'matched' to the neural representation of the corresponding (yet unseen) actions executed earlier. Secondly, we wanted to assess the relation between the amount of physical practice which had been invested to learn each of the movement sequences and the activation levels of the involved brain regions during their observation. Such an experiencedependent modulation would suggest that the observer's own motor repertoire influences the way in which (the perceptual consequences of) observed actions are perceived. The displayed motion paths were based on movement sequences which the subjects had previously learned by continuously tracing a set of predefined 2-dimensional maze trajectories (Figure 1A; [8-10]). In a first scanning session, two maze trajectories were introduced. Afterwards, one of them was extensively trained over three days in order to allow a comparison between both trajectories in terms of invested training during a second scanning session four days later. Importantly, the required tracing movements were performed with closed eyes and without having seen the trajectories beforehand. Consequently, subjects could only rely on somatosensory cues and proprioceptive feedback while learning the movement sequences, by tracing through a groove to which their pen movements were restricted. Subjects were subsequently asked to discriminate between the traced trajectories and similar non-encountered trajectories in a visually presented matching task (Figure $1 \mathrm{~B} / \mathrm{C})$. By including both the actual execution and the observation of the same actions, the applied design allowed to assess the specificity of the recorded neural responses. Additionally, any effects of visual familiarity on observationrelated activations could be ruled out because the paradigm only involved nonvisual motor learning conditions.

\section{Motor Learning}

A set of Regions of Interest (ROIs) was functionally defined based on the maze tracing data from the first scanning session, by contrasting motor learning conditions (in which novel maze trajectories were traced) with appropriate control conditions (in which a familiar square-shaped trajectory was traced either fast or slowly and no additional learning occurred). The identified ROIs included: the bilateral dorsal (PMd) and ventral (PMv) premotor cortex, the inferior postcentral sulcus (InfPCS), the anterior intraparietal sulcus (aIPS), the 


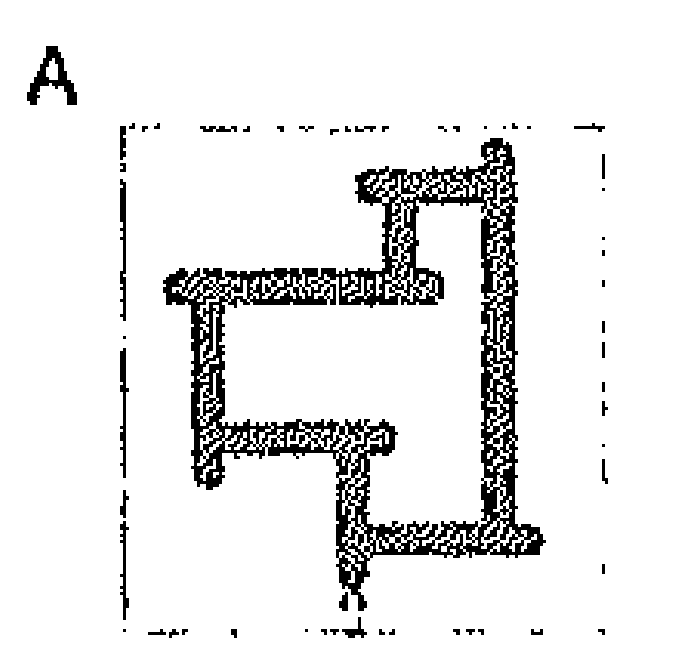

$\mathrm{B}$
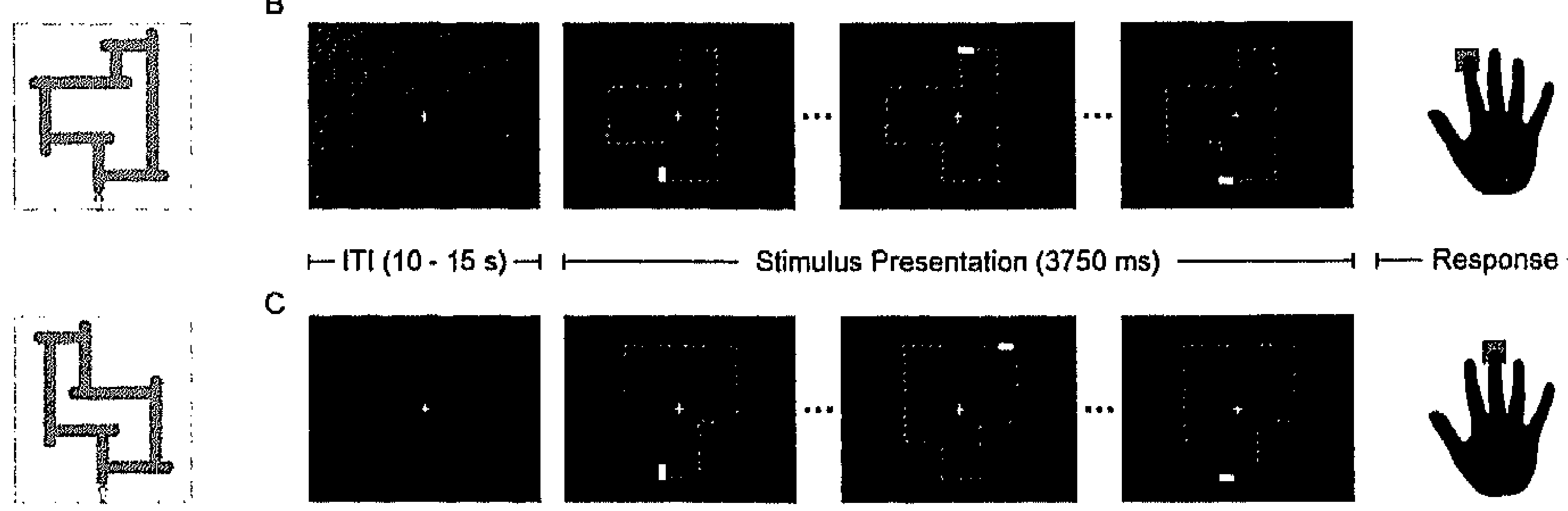

Figure 1. Traced trajectories and visual matching design

(A) One of the movement sequences represented by the mazes shown here was extensively trained over the course of several days in between two scanning sessions. The white arrows at the bottom indicate the starting points, and in both cases the total trajectory was $260 \mathrm{~mm}$ long. Note that the correct movement sequences needed to be learned by trial and error, in the absence of any visual feedback. (B) An example of a trial as presented during the visual matching task, in which a matching trajectory was displayed. After a variable Inter-Trial Interval (ITI), a small white rectangle started to move along an invisible path (depicted by the green dashed line). Subjects were instructed to covertly follow the movements of the rectangle (while fixating the fixation cross), and online compare its trajectory with the movement sequences they had previously performed themselves. Once the rectangle arrived at its starting point again, subjects should indicate whether the observed trajectory matched with one of the performed movement sequences or not (via a forced-choice button press). (C) A similar example, but now showing a nonmatching trajectory and the response which should be given correspondingly.

posterior superior parietal lobule (SPL), and a region in the left medial IPS/posterior SPL (see Figure 2A). Note that these ROIs did not differentiate between the fast and slow square tracing conditions when contrasting these control conditions directly, implying that the observed activations truly reflected learning (i.e. instead of the accompanying increase in tracing velocity). This was furthermore corroborated by the finding that the specified ROIs changed their activation levels as learning progressed, showing learning-related decreases both within and across sessions. 

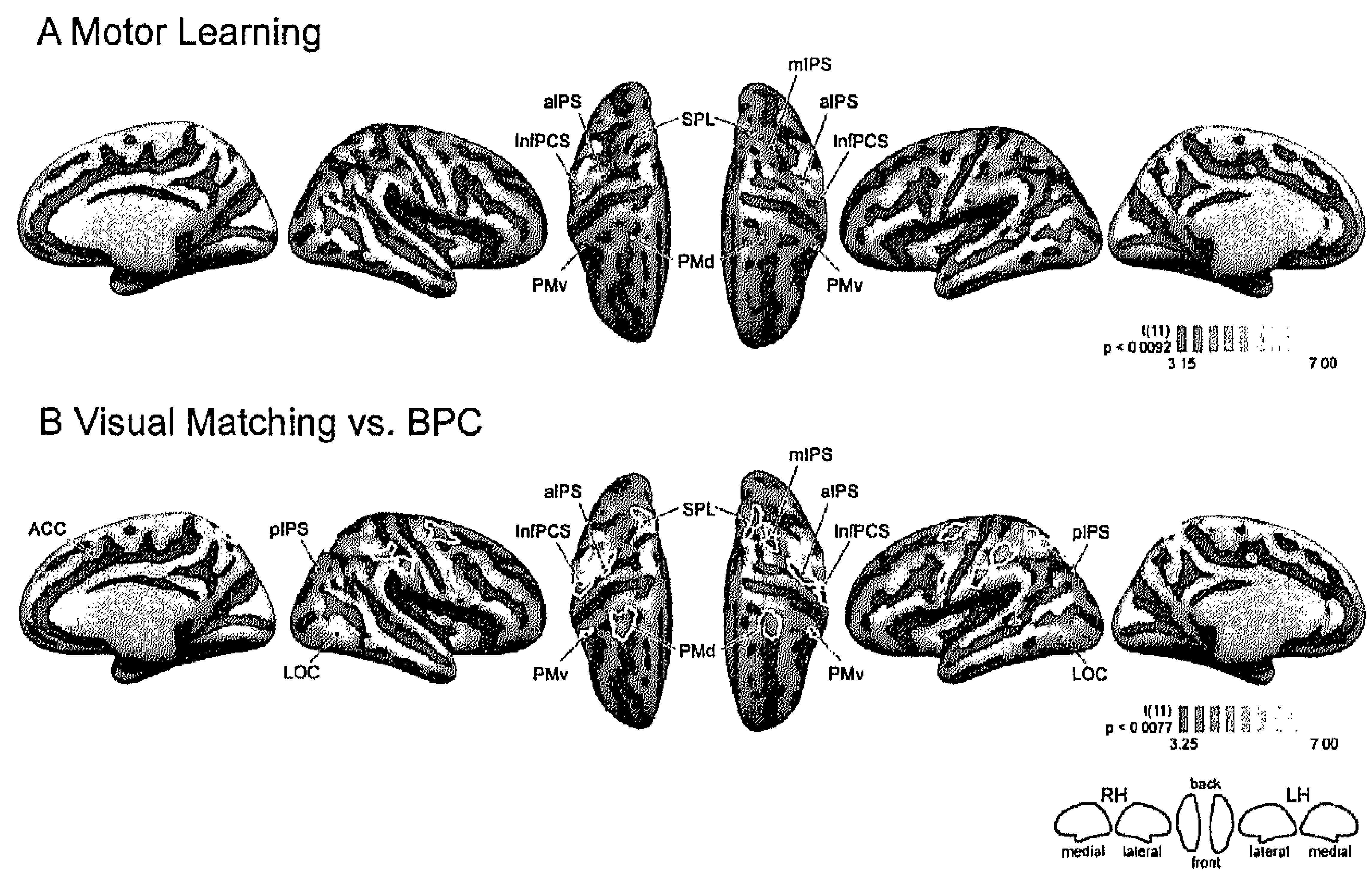

Figure 2. Group activation maps showing regions implicated in motor learning and their reactivation during visual matching

(A) The network of learning-related areas as identified during the maze tracing task in scanning session 1, projected on the inflated version of the average group cortex mesh (random effects; $\mathrm{t}(11)=3.15 ; \mathrm{p}$ (uncorr) $<0.0092$ ). The implicated areas showed stronger responses when subjects learned to execute the novel movement sequences, compared to the control conditions in which a known square was traced either fast or slowly. (B) The displayed map shows the regions which were significantly more active during the visual matching task compared to the button press control (BPC) task. Importantly, all learning-related ROIs (depicted by white contours) which were defined during the physical execution of the corresponding movement sequences were reactivated again. Note that the currently described analyses focused on the data from session two, because of the relatively large fraction of incorrectly classified trajectories in session one (see Figure S1). Abbreviations: PMd, dorsal premotor cortex; PMv, ventral premotor cortex; InfPCS, inferior postcentral sulcus; aIPS, anterior intraparietal sulcus; mIPS; medial intraparietal sulcus; SPL, superior parietal lobule; ACC, anterior cingulate cortex; pIPS, posterior intraparietal sulcus; LOC, lateral occipital complex; $\mathrm{RH}$, right hemisphere; $\mathrm{LH}$, left hemisphere.

\section{Visual Matching}

For the visual matching task, a conjunction of the different trial types separately contrasted with the inter-trial baseline revealed activations in a widely distributed network of areas, which was highly replicable across sessions (Figure S2). Interestingly, performing the visual matching task reactivated the brain areas specifically implicated in learning the novel motor routines, even though subjects did not execute any overt movement sequences at that point. To rule out that the observed reactivations were simply related to the production of 
the required button presses, the data from the 'button press control' task (BPC; see Experimental Procedures) were contrasted with the responses obtained during the matching task. The resulting activation map is depicted in Figure 2B and still clearly includes all learning-related areas (marked by white contours). The role of the learning-related areas in matching was also confirmed by additional random effects analyses at the ROI level indicating that the responses during the matching task exceeded the responses in the BPC task in all considered ROIs (as exemplified in Figure 3).
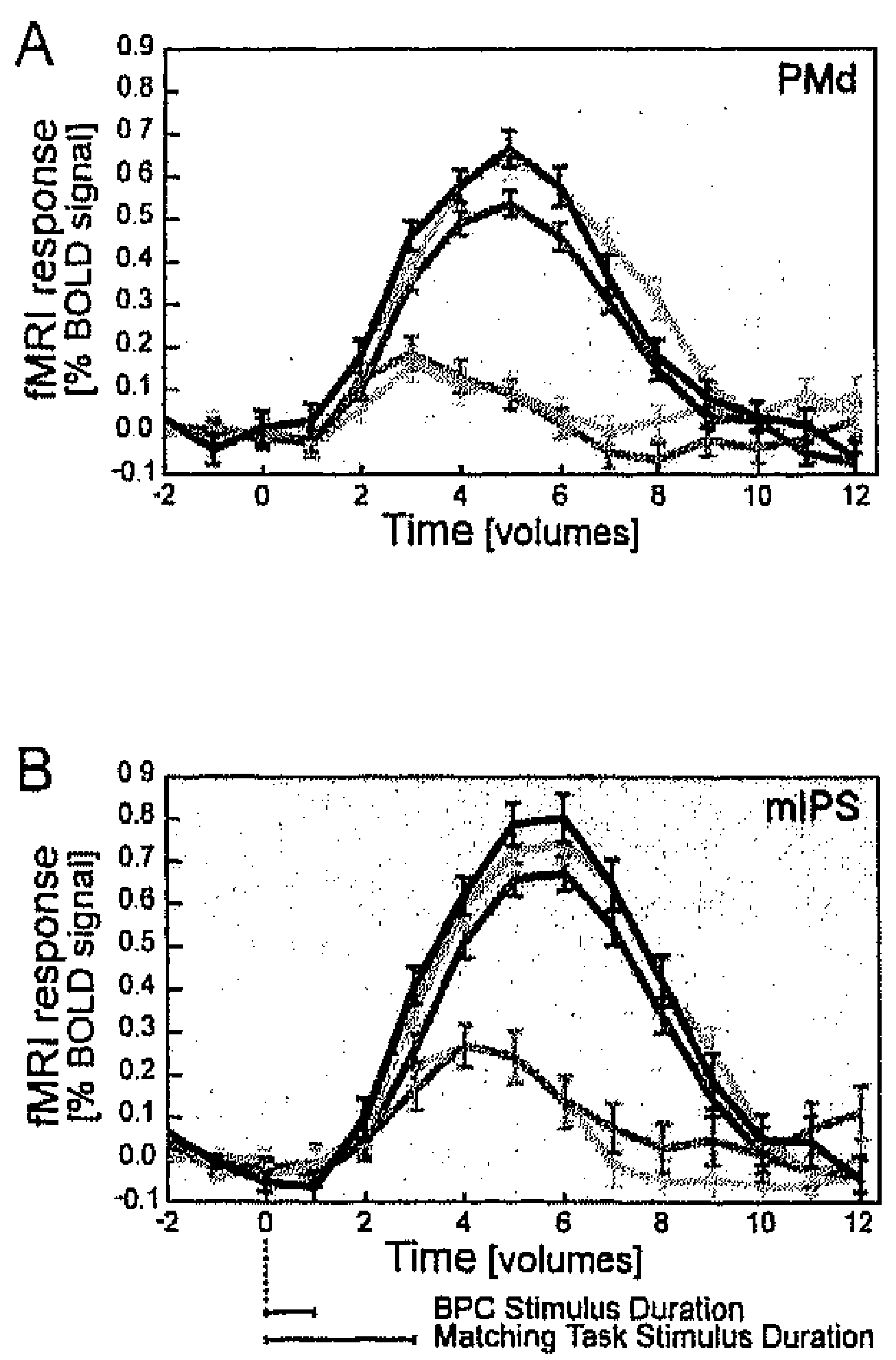
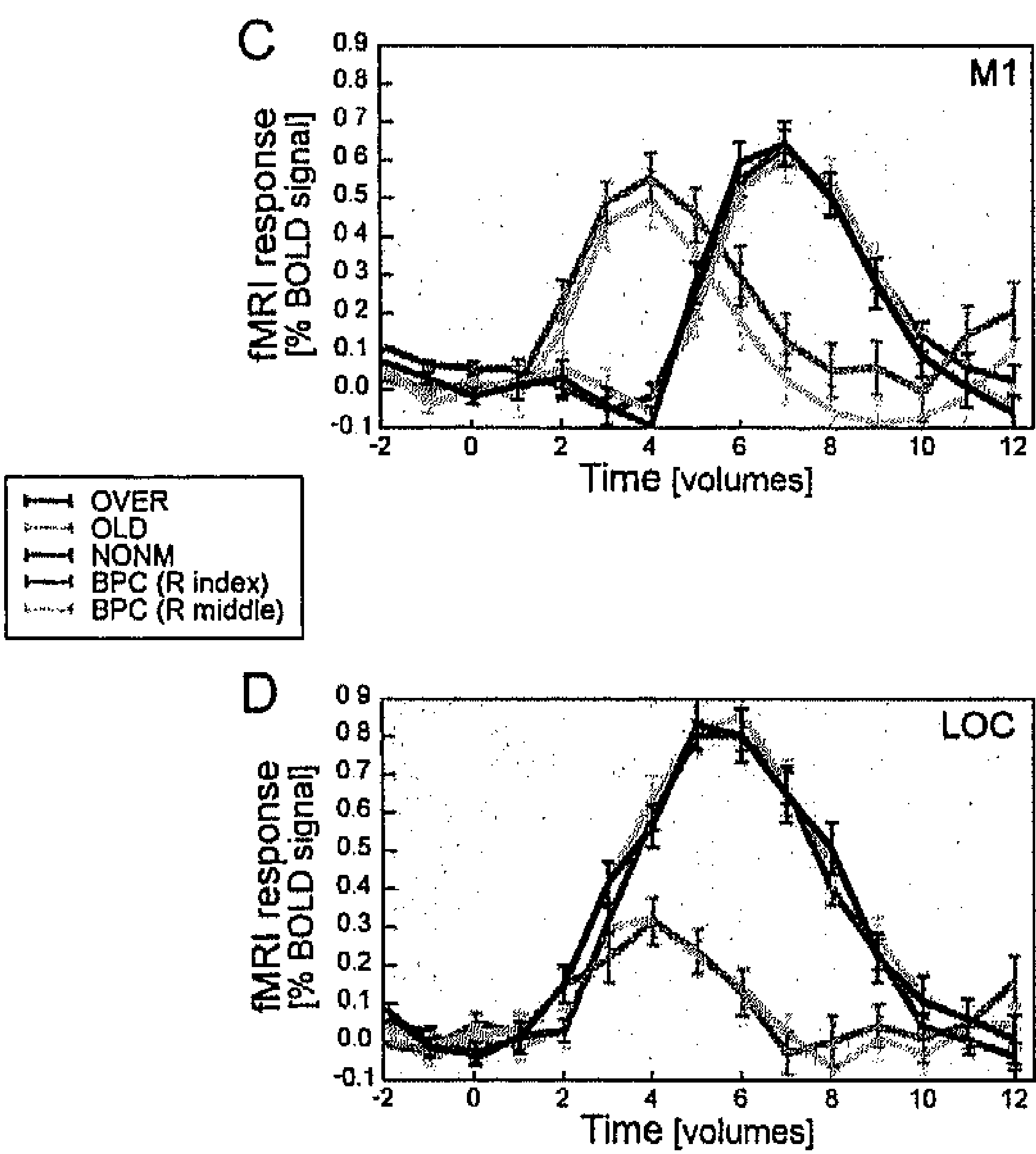

Figure 3. Representative event-related responses during visual matching

This figure shows event-related responses averaged across subjects for (A) the left $\mathrm{PMd},(\mathrm{B})$ the left mIPS, (C) the left primary motor cortex (M1), and (D) the left Lateral Occipital Complex (LOC). Error bars represent standard errors of the mean. Note that in (A), (B) and (D), the responses during the matching task by far exceed the activation amplitude associated with the BPC task. In contrast, the responses shown in (C) are similar in both conditions and the shift in onset during the visual matching task clearly indicates their relation to the required button press, Furthermore, only the PMd and mIPS ROIs show an enhanced response during the observation of matching compared to non-matching trajectories. Finally, the (non-differential) responses observed in the LOC are depicted to illustrate that the divergent response pattern in the learningrelated ROIs is not confounded by differences in the general amount of attention deployed to matching and non-matching trajectories. Abbreviations: 'OVER', overlearned sequence; 'OLD', previously encountered but not extensively trained sequence; 'NONM', non-matching trajectory; 'BPC', button press control task; ' $R$ ', right hand. 
Separate random effects ROI GLMs on the data from the second scanning session revealed that a large majority of the learning-related areas differentiated between matching and non-matching trajectories, by responding more strongly to the displayed trajectories matching the actively learned and repeatedly performed sequences (Table 1 and Figure 3A/B).

\begin{tabular}{|c|c|c|c|c|c|c|c|c|c|}
\hline \multirow{3}{*}{$\begin{array}{l}\text { Region } \\
\text { of } \\
\text { Interest }\end{array}$} & \multirow[t]{3}{*}{$\begin{array}{l}\text { Hemi- } \\
\text { sphere }\end{array}$} & \multirow{2}{*}{\multicolumn{3}{|c|}{$\begin{array}{l}\text { Talairach } \\
\text { Coordinates } \\
\qquad \mathrm{mm} \mid\end{array}$}} & \multicolumn{5}{|c|}{$\begin{array}{l}\text { Significance of Differential Responses } \\
\text { t"values and probabilities for contrasts (RFX ROI-(LLM; } n=12)\end{array}$} \\
\hline & & & & & $\begin{array}{l}\text { OVFR } \\
\text { OLD }=\text { BPC } \\
\text { NONM }\end{array}$ & OVER $=$ NONM & OVER $>$ NONN & OVER $\therefore$ OLD & OLD $>$ NONM \\
\hline & & $\mathrm{x}$ & $y$ & $\mathrm{z}$ & $t \quad(p)$ & (p) & $(p)$ & (p) & (p) \\
\hline PMd & $\begin{array}{l}\text { LH } \\
\text { RH }\end{array}$ & $\begin{aligned}-21 \\
25\end{aligned}$ & $\begin{array}{l}-9 \\
-8\end{array}$ & $\begin{array}{l}52 \\
48\end{array}$ & $\begin{array}{l}6.92(0.000)^{* *} \\
6.94(0.000)^{* *}\end{array}$ & $\begin{array}{l}3.33(0.003)^{* *} \\
2.04(0.033)^{*}\end{array}$ & $\frac{2.84(0.008)^{*}}{2.35(0.019)^{*}}$ & $\begin{array}{l}0.72(0.242) \\
1.60(0.069)\end{array}$ & $\begin{array}{l}2.57(0.013)^{\prime} \\
0.9+(0.185)\end{array}$ \\
\hline PMV & $\begin{array}{l}\mathrm{LH} \\
\mathrm{RH}\end{array}$ & -52 & $\begin{array}{r}-1 \\
2\end{array}$ & $\begin{array}{l}34 \\
34\end{array}$ & $\begin{array}{l}6.30(0.000)^{* *} \\
6.31(0.000)^{* *}\end{array}$ & $\begin{array}{l}2.19(0.026)^{*} \\
1.53(0.078)\end{array}$ & $\begin{array}{l}2.98(0.006)^{*} \\
1.70(0.058)\end{array}$ & $\begin{array}{l}1.62(0.067) \\
0.76(0.233)\end{array}$ & $\begin{array}{l}0.44(0.335) \\
0.22(0.415)\end{array}$ \\
\hline InfPCS & $\begin{array}{l}\text { LH } \\
\text { RH }\end{array}$ & $\begin{array}{r}-54 \\
49\end{array}$ & & 32 & $\begin{array}{l}5.15(0.000)^{* *} \\
6.88(0.000)^{* *}\end{array}$ & $\begin{array}{l}3.13(0.005)^{*} \\
3.93(0.001)^{* *}\end{array}$ & $\begin{array}{l}3.86(0.001)^{* *} \\
4.91(0.000)^{* *}\end{array}$ & $\begin{array}{l}1.91(0.041)^{*} \\
1.17(0.134)\end{array}$ & $\begin{array}{l}0.84(0.209) \\
1.54(0.076)\end{array}$ \\
\hline aIPS & $\begin{array}{l}\text { LH } \\
\text { RHI }\end{array}$ & $\begin{array}{r}-39 \\
36\end{array}$ & & $\begin{array}{l}42 \\
39\end{array}$ & $\begin{array}{l}6.57(0.000)^{* *} \\
8.74(0.000)^{* *}\end{array}$ & $\begin{array}{l}2.71(0.010)^{*} \\
3.55(0.002)^{* *}\end{array}$ & $\begin{array}{l}3.44(0.003)^{* *} \\
2.55(0.014)^{*}\end{array}$ & $\begin{array}{l}1.61(0.068) \\
0.09(0.467)\end{array}$ & $\begin{array}{l}1.02(0.165) \\
2.26(0.023)^{*}\end{array}$ \\
\hline mIPS & $\begin{array}{l}\mathrm{LH} \\
\mathrm{RH}\end{array}$ & -26 & -53 & $5 !$ & $8.03(0.000)^{k *}$ & $5.53(0.000)^{* *}$ & $4.97(0.000)^{* *}$ & $\begin{array}{c}1.06(0.156) \\
-\end{array}$ & $\begin{array}{c}1.75(0.054) \\
-\end{array}$ \\
\hline SPL & $\begin{array}{l}\mathrm{LH} \\
\mathrm{RH}\end{array}$ & $\begin{array}{r}-17 \\
13\end{array}$ & $\begin{array}{l}-66 \\
-59\end{array}$ & $\begin{array}{l}52 \\
53\end{array}$ & $\begin{array}{l}5.37(0.000)^{* *} \\
6.92(0.000)^{* *}\end{array}$ & $\begin{array}{l}3.79(0.002)^{* *} \\
2.56(0.013)^{*}\end{array}$ & $\begin{array}{l}4.10(0.001))^{* *} \\
2.90(0.007)^{*}\end{array}$ & $\begin{array}{l}0.83(0.213) \\
1.00(0.170)\end{array}$ & $\begin{array}{l}1.78(0.051) \\
1.09(0.149)\end{array}$ \\
\hline
\end{tabular}

Table 1. Results from the random effects ROI analyses related to the visual matching task data from session 2

The table lists the relevant contrasts related to the visual matching task for all included ROIs. Note that all ROIs were functionally defined in the first scanning session based on their involvement in learning to perform the novel movement sequences. The results indicate that: (1) all ROIs showed enhanced responses during the matching task as compared to the "button press control' task; (2) a consistently stronger response when observing the overlearned sequence (compared to non-matching trajectories) was found across all ROIs (except right PMv). Even though the 'OVER>OLD' contrast was only on the verge of significance in a subset of the studied ROIs, a clear activation pattern became evident in which the response to the OLD sequence reached an intermediate position between the OVER and NONM responses, as depicted in Figure 4. Note that the listed p-values are based on one-tailed t-tests.

* $\mathrm{p}<0.05$ (uncorrected); ${ }^{* *} \mathrm{p}<0.0045$ (Bonferroni-corrected for the number of included ROIs). Abbreviations as in Figures 2/3.

Moreover, as additional analyses in which the overlearned ('OVER') and less extensively trained ('OLD') sequences were separately contrasted with the nonmatching stimuli established, this effect was mainly caused by a stronger response to the overlearned sequence (Table 1; third contrast). Except for the right $\mathrm{PMv}$, all areas implicated in learning tended to respond more vigorously whenever a trajectory was presented which matched the extensively trained sequence (Figure 4). Because the sequence which was overlearned was 
counterbalanced across subjects, these enhanced responses cannot be related to a specific set of sequential movements per se, but really reflect the overlearned status of the corresponding movement pattern. Finally, the observation that differential response patterns for matching and non-matching trajectories were restricted to the learning-related motor areas and were not found within a wider set of regions (e.g., the Lateral Occipital Complex (LOC), see Figure 3D), suggests that this divergence is not related to a general 'de-allocation' of attention in the case of the non-matching stimuli.
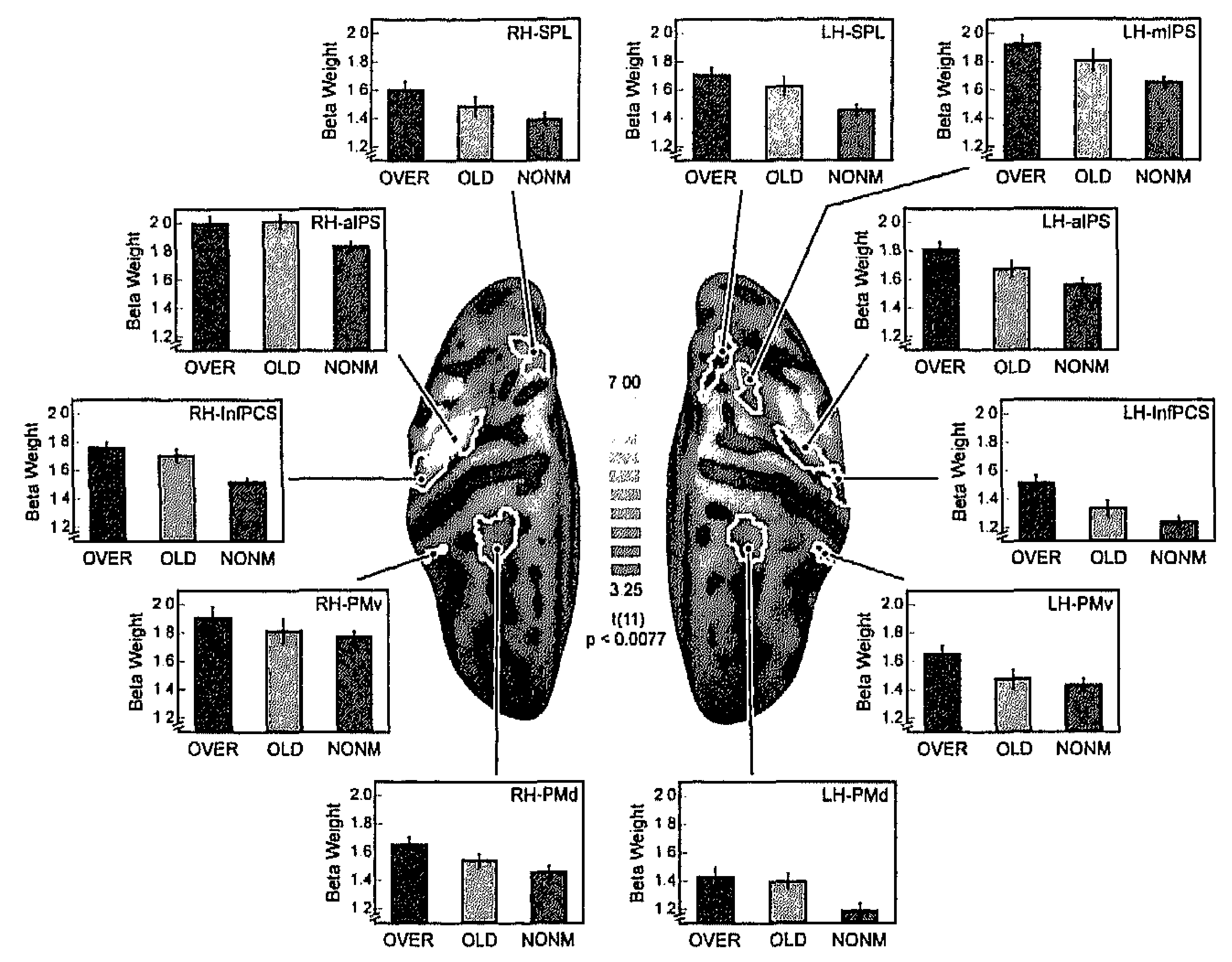

Figure 4. Overview of the responses to the different visual matching conditions for all identified ROIs

This figure gives an overview of the activation levels across the different visual matching conditions for all functionally defined ROIs, based on the estimated beta-weights. Note that in nearly all ROIs, a similar pattern emerges in which a path matching the overlearned sequence gives rise to the strongest response, whereas a non-matching trajectory produces the weakest activations. Error bars indicate the standard error of the mean following a normalization procedure in which the grand mean across subjects and conditions was first subtracted from each subject's own mean beta across conditions, and subsequently this resulting value was subtracted from the subject-specific beta's per condition. Abbreviations as in Figures 2/3. 
Action observation

\section{Discussion}

In the current study, subjects were confronted with the visual counterpart of previously performed, but unseen movement sequences. The dynamic nature of the presented visual stimuli and the feature that previous positions along the path were not highlighted (i.e., no trace was visible), probably reinforced the use of the already stored motor representations to accomplish the outlined task. Interestingly, the areas which were specifically involved in learning to execute the trained sequences were recruited again while performing the visual matching task, as part of a more extended network including areas previously shown to be implicated in for example the multimodal processing of object shapes (LOC; [11]) and covert spatial attention (e.g., pIPS; [12]). In part, the observed reactivations comprised regions which have been typically associated with mirror system functions, such as the PMv $[13,14]$ and the InfPCS, which following [15] might at the cytoarchitectonic level be considered part of the anterior inferior parietal lobule [16]. On the other hand, several other regions (PMd, aIPS, mIPS and SPL) were additionally involved in the matching task, supporting the emerging view that a more widespread network of regions is implicated during action observation (see also [17-19]). These findings were highly replicable both across subjects and across sessions. Moreover, it was shown that the potential confounding effect of having to respond via button presses (which by itself might explain the involvement of motor-related areas), could not account for the observed reactivations. Rather, the involvement of the learning-related motor areas suggests that observing a novel visual representation of the previously performed movements goes beyond passive sensory processing and involves an active simulation of the corresponding motor act.

This interpretation is in accordance with the general notion that nonvisual motor training can enhance visual perception capabilities (e.g., [20]) and previous neurophysiological data showing that neurons in the monkey PMd discharge both when actually performing a reaching task and while only observing the visual events associated with the task when performed by another unseen party [21]. Additionally, these PMd neurons started to discharge even before any visual motion could be detected once enough information was provided regarding which event could be expected next. This preparatory activity highlights that the responses recorded during the observation condition were not purely 'reactive', but part of an active simulation process. Similarly, such proactive responses have been reported in humans using eye movement recordings [22] and electroencephalogram measurements [23]. Another interesting aspect of the reported study is related to the nature of the used stimuli. Although there is still an ongoing debate concerning the question whether the human mirror system is 'biologically tuned' in the sense that it 
preferentially responds to natural movements performed by other human beings [e.g., 19,24-26], the present results suggest that even rather abstract visual stimuli conveying minimal information concerning the underlying movement can trigger the corresponding motor representations.

The obtained results additionally are in line with the hypothesis that the extent to which a given stimulus succeeds in activating the mirror system depends on the existing motor repertoire of the observer (e.g., see [27]). Two interesting fMRI studies involving professional dancers recently showed that an observer's proficiency at performing certain movements influences the neural responses elicited by the sight of the same movements being performed by somebody else $[28,29]$. There are several commonalities between these imaging experiments and the study presented here. First, as pointed out in [29], earlier studies on action simulation required subjects to imagine a given action without any external guidance. Instead, the provided visual stimuli in the studies discussed here were intended to guide and constrain the ongoing simulation, leading to a more homogeneous sampling of the process of interest. Additionally, the simulation was behaviorally controlled in the present study, by including a forced-choice matching task which enabled the evaluation of the subjects' simulation performance post hoc. Secondly, all three studies lend further support to the notion that the observation-guided simulation of motor sequences relies on some of the same areas which are also active during the overt execution of the corresponding movements. However, in contrast to both dance observation studies, this equivalence was explicitly established in the present study by including appropriate motor learning conditions. Third, and in line with other imaging results [e.g., 18, 19], all three studies suggest that simulating perceived actions involves areas for which the assessment of mirror responses at the single-cell level is still lacking to date. Finally, the listed findings are in agreement with the hypothesis that the neural activity which is related to the simulation of an observed action is modulated by the experience of the observer, in the sense that formerly trained (i.e., embodied) action sequences lead to stronger activations. The current study extends previous findings regarding this latter issue in two ways. Firstly, the level of expertise of the subjects in the current study was objectively assessed and quantified by monitoring their performance during the actual production of the movement sequences. Moreover, it could be shown that the very same regions were recruited in both the early and late stages of learning, ensuring that the equivalence between the networks observed during motor execution and visual matching was preserved throughout the learning process. The second point is related to the visual familiarity of the presented stimuli. Complementary to the findings from an elegant follow-up study by Calvo-Merino et al. [30], the current results show that visual familiarity cannot constitute a confounding 
factor when interpreting the observed experience-dependent modulations, given that the displayed stimuli corresponded to previously unseen movements.

In sum, the presented results indicate that when perception guides the internal simulation of previously executed (yet unseen) movement sequences, a distributed network of areas specifically involved in learning to overtly execute these movements will be recruited again. Furthermore, the degree to which these learning-related regions are reactivated is dependent on how well the observed information matches the observer's direct experience in performing the corresponding movement. Since the used matching task necessitates the continuous online simulation of an acquired movement sequence, the processes it taps into might be more tightly coupled to the matching of sensory events to stored motor representations than the more end-goal driven coding as observed in the classically defined mirror neuron system in monkeys.

\section{Experimental Procedures}

\section{Participants}

Twelve healthy volunteers with normal visual acuity ( 6 males; mean age $=25$ years, standard deviation $=1.4$ years) participated in the current study. All subjects gave written informed consent following procedures approved by the local research ethics committee. Right-handedness was used as an inclusion criterion and was assessed using the Edinburgh Handedness Inventory [31] (mean score $=94$, standard deviation $=9$ ).

\section{Experimental Paradigm}

All subjects participated in two separate scanning sessions, both consisting of two main parts: a motor learning task, in which different movement sequences were learned and repeatedly executed with closed eyes (see Supplemental Data for details), and a subsequent visual matching task, in which subjects were asked to discriminate between the actually traced trajectories and other nonmatching trajectories based on a visually presented representation of the corresponding movements. In both scanning sessions, the visual matching task immediately followed the motor learning task within the same functional run. Throughout the matching task, a fixation cross was shown in the middle of the display. In each trial, a small white rectangle appeared and started to continuously move along an invisible trajectory until ending up at its starting position again. There were two types of trajectories: the path that the white rectangle followed either coincided with one of the movement sequences previously executed by the subject (Figure 1B), or represented one of four 
Chapter 4

different sequences which were not encountered before (Figure 1C). Subjects were instructed to pay attention to the entire trajectory that the white rectangle traversed, without moving their eyes away from the fixation cross. Their task was to decide whether the presented trajectory constituted a match or a nonmatch with one of the previously performed movement sequences. In order not to contaminate the neural responses to the visually presented trajectory with the activations related to the button presses, subjects were furthermore instructed to wait until the white rectangle reached its starting position again, before responding.

Finally, an additional button press control (BPC) task including visually-cued button presses was performed in the second scanning session to rule out that potential activations in areas related to motor learning might be present due to the use of button presses as behavioral responses (see Supplemental Data).

\section{Data Acquisition \& Analysis}

The (f)MRI data were acquired on a 3T Siemens Magnetom Allegra headscanner (Siemens Medical Systems, Erlangen, Germany) and analyzed using BrainVoyager QX (v1.7; Brain Innovation, Maastricht, The Netherlands). See Supplemental Data for details on scan parameters, preprocessing, the applied cortex-based alignment procedures and subsequent random-effects statistical analyses.

\section{Acknowledgements}

The authors are grateful to Bettina Sorger for her help throughout the different stages of this project. 


\section{References}

1. Rizzolatti, G., \& Craighero, L. (2004). The mirror-neuron system. Annual Review of Neuroscience, 27, 169-192.

2. Rizzolatti, G., Fadiga, L., Matelli, M., Bettinardi, V., Paulesu, E., Perani, D., \& Fazio, F. (1996). Localization of grasp representations in humans by PET: 1. Observation versus execution. Experimental Brain Research, 111, 246-252.

3. Iacoboni, M., Woods, R.P., Brass, M., Bekkering, H., Mazziotta, J.C., \& Rizzolatti, G. (1999). Cortical mechanisms of human imitation. Science, 286, 2526-2528.

4. Buccino, G., Binkofski, F., Fink, G.R., Fadiga, L., Fogassi, L., Gallese, V., Seitz, R.J., Zilles, K., Rizzolatti, G., \& Freund, H-J. (2001). Action observation activates premotor and parietal areas in a somatotopic manner: an AMRI study. European Journal of Neuroscience, 13, 400-404.

5. Grezes, J., Armony, J.L., Rowe, J., \& Passingham, R.E. (2003). Activations related to "mirror" and "canonical" neurons in the human brain: an fMRI study. NeuroImage, 18, 928-937.

6. Iacoboni, M., Molnar-Szakacs, I., Gallese, V., Buccino, G., Mazziotta, J.C., \& Rizzolatti, G. (2005). Grasping the intentions of others with one's own mirror neuron system. PLoS Biology, 3, 529-535.

7. Rizzolatti, G., Fogassi, L., \& Gallese, V. (2001). Neurophysiological mechanisms underlying the understanding and imitation of action. Nature Reviews Neuroscience, 2 , 661-670.

8. van Mier, H., Tempel, L.W., Perlmutter, J.S., Raichle, M.E., \& Petersen, S.E. (1998). Changes in brain activity during motor learning measured with PET: effects of hand of performance and practice. Journal of Neurophysiology, 80, 2177-2199.

9. van Mier, H.I., Perlmutter, J.S., \& Petersen, S.E. (2004). Functional changes in brain activity during acquisition and practice of movement sequences. Motor Control, 8, 500520.

10. Reithler, J., Reithler, H., van den Boogert, E., Goebel, R., \& van Mier, H. (2006). Resistance-based high resolution recording of predefined 2-dimensional pen trajectories in an fMRI setting. Journal of Neuroscience Methods, 152, 10-17.

11. Amedi, A., Malach, R., Hendler, T., Peled, S., \& Zohary, E. (2001). Visuo-haptic object-related activation in the ventral visual pathway. Nature Neuroscience, 4, 324330. 


\section{Chapter 4}

12. Jovicich, J., Peters, R.J., Koch, C., Braun, J., Chang, L., \& Ernst, T. (2001). Brain areas specific for attentional load in a motion-tracking task. Journal of Cognitive Neuroscience, 13, 1048-1058.

13. Johnson-Frey, S.H., Maloof, F.R., Newman-Norlund, R., Farrer, C., Inati, S., \& Grafton, S.T. (2003). Actions or hand-object interactions? Human inferior frontal cortex and action observation. Neuron, 39, 1053-1058.

14. Binkofski, F., \& Buccino, G. (2006). The role of ventral premotor cortex in action execution and action understanding. Journal of Physiology Paris, 99, 396-405.

15. Caspers, S., Geyer, S., Schleicher, A., Mohlberg, H., Amunts, K., \& Zilles, K. (2006). The human inferior parietal cortex: Cytoarchitectonic parcellation and interindividual variability. NeuroImage, 33, 430-448.

16. Gallese, V., Fadiga, L., Fogassi, L., \& Rizzolatti, G. (2002). Action representation and the inferior parietal lobule. In W. Prinz \& B. Hommel (Eds.), Common mechanisms in perception and action (pp. 334-355). New York, NY: Oxford University Press.

17. Shmuelof, L., \& Zohary E. (2006). A mirror representation of others' actions in the human anterior parietal cortex. Journal of Neuroscience, 26, 9736-9742.

18. Frey, S.H., \& Gerry, V.E. (2006). Modulation of neural activity during observational learning of actions and their sequential orders. Journal of Neuroscience, $26,13194-13201$.

19. Gazzola, V., Rizzolatti, G., Wicker, B., \& Keysers, C. (2007). The anthropomorphic brain: The mirror neuron system responds to human and robotic actions. Neurolmage, $35,1674-1684$.

20. Casile, A., \& Giese, M.A. (2006). Nonvisual motor training influences biological motion perception. Current Biology, 16, 69-74.

21. Cisek, P., \& Kalaska, J.F. (2004). Neural correlates of mental rehearsal in dorsal premotor cortex. Nature, 431, 993-996.

22. Flanagan, J.R., \& Johansson, R.S. (2003). Action plans used in action observation. Nature, 424, 769-771.

23. Kilner, J.M., Vargas, C., Duval, S., Blakemore, S-J., \& Sirigu, A. (2004). Motor activation prior to observation of a predicted movement. Nature Neuroscience, 7, 12991301.

24. Kilner, J.M., Paulignan, Y., \& Blakemore, S.J. (2003). An interference effect of observed biological movement on action. Current Biology, 13, 522-525. 
25. Tai, Y.F., Scherfler, C., Brooks, D.J., Sawamoto, N., \& Castiello, U. (2004). The human premotor cortex is 'mirror' only for biological actions. Current Biology, 14, 117 120.

26. Press, C., Bird, G., Flach, R., \& Heyes, C. (2005). Robotic movement elicits automatic imitation. Cognitive Brain Research, 25, 632-640.

27. Buccino, G., Lui, F., Canessa, N., Patteri, I., Lagravinese, G., Benuzzi, F., Porro, C.A., \& Rizzolatti, G. (2004). Neural circuits involved in the recognition of actions performed by non-conspecifics: an AMRI study. Journal of Cognitive Neuroscience, 16, $1-14$.

28. Calvo-Merino, B., Glaser, D.E., Grezes, J., Passingham, R.E., \& Haggard, P. (2005). Action observation and acquired motor skills: An AMRI study with expert dancers. Cerebral Cortex, 15, 1243-1249.

29. Cross, E.S., Hamilton, A.F.C., \& Grafton, S.T. (2006). Building a motor simulation de novo: Observation of dance by dancers. Neurolmage, 31, 1257-1267.

30. Calvo-Merino, B., Grezes, J., Glaser, D.E., Passingham, R.E., \& Haggard, P. (2006). Seeing or doing? Influence of visual and motor familiarity in action observation. Current Biology, 16, 1905-1910.

31. Oldfield, R.C. (1971). The assessment and analysis of handedness: the Edinburgh Inventory. Neuropsychologia, 9, 97-113. 


\section{Supplemental Data}

\section{Supplemental Results}

\section{Behavioral Matching Performance}

In the first scanning session, the overall percentage of correctly classified trials was $77.1 \% \pm 3.4 \%$ (mean \pm standard error), when pooling across all stimulus types. However, when specified according to the different stimulus types, the following pattern emerged: whereas $87.5 \pm 3.9 \%$ of the non-matching trials were correctly identified as such, the two matching trajectories were correctly classified in $60.0 \pm 6.7 \%$ and $65.8 \pm 7.2 \%$ of the cases, respectively. This is also depicted on the left side of the bar graph in Figure S1. Note that the labeling of the matching trajectories in the first session was based on whether a specific sequence would be extensively trained during the following days ('OVER' for 'overlearned') or not ('OLD'), even though both sequences were encountered for the first time in the initial fMRI session. A one-way repeated measures ANOVA with stimulus type as within-subjects factor showed that the three reported means diverged significantly $\left(\mathrm{F}_{(2,22)}=6.2 ; \mathrm{p}=0.007\right)$, and subsequent pair-wise comparisons revealed that the percentage of correct responses for the non-matching trials was significantly higher compared to the percentage for both matching trajectories $(\mathrm{p}<0.05$ after Bonferroni correction for multiple comparisons).

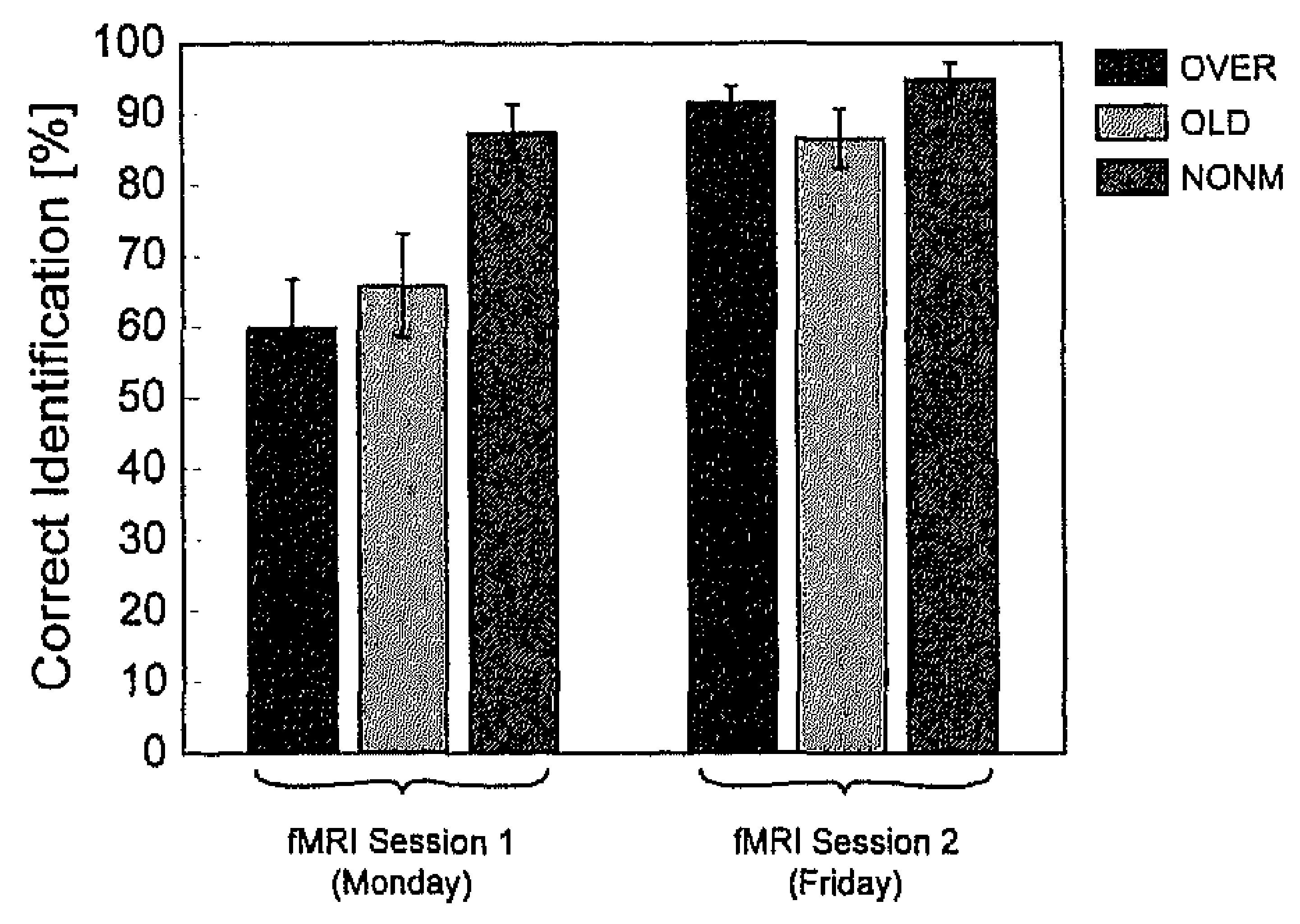

Figure S1. Behavioral results for the visual matching task

The bar graph shows the mean percentage of correctly identified trajectories with the corresponding standard error across subjects $(n=12)$, split according to trial type and scanning session. Note that although both performed sequences were new to the subjects in the first fMRI session, their labels are based on the description of their functional role in the second scanning session ('OVER' for the overlearned sequence which was extensively trained on the days between 
both AMRI sessions, and 'OLD' for the sequence which was already encountered in the first scanning session, but was not practiced afterwards). The 'NONM' label collectively refers to the four non-matching trajectories which were correctly identified as such. The data show that subjects performed better for the non-matching than for the matching trials in the first session, whereas no difference between the trial types was observed in the second scanning session.

The overall matching performance during the second scanning session was characterized by a total of $92.3 \pm 2.0 \%$ correct responses. The overlearned sequence was correctly identified in $91.7 \pm 2.4 \%$ of the cases, a match between the 'OLD' sequence and the presented trajectory was correctly indicated in 86.7 $\pm 4.1 \%$ of the trials, and $95.0 \pm 2.3 \%$ of the non-matching trajectories were correctly classified as such. The results from an one-way repeated measures ANOVA with stimulus type as within-subjects factor indicated that the performance was at a similar level for all three trial types $\left(\mathrm{F}_{(2,22)}=2.2 ; \mathrm{p}=\right.$ 0.138). In the 'button press control' task (BPC, see Experimental Procedures), which was additionally performed to rule out that any potentially observed reactivations in motor regions could be explained by the necessity of a button press in the matching task, the correct button was pressed $98.4 \pm 1.6 \%$ of the time.

\section{Imaging Data}

The results of the conjunction analyses in which all the different matching trial types were separately contrasted to the inter-trial baseline are displayed in Figure S2. The obtained maps show the regions which were involved in the matching task, irrespective of the type of trajectory that was presented. Note that all identified motor learning regions are reactivated during the visual matching task, and that the identified network is highly consistent across both scanning sessions. Subsequent random-effects ROI analyses confirmed these results (resulting in highly significant $t$-values ranging from $t=6.7$ to $t=14.7$ ). The most prominent additional responses were found bilaterally in the lateral occipital complex (LOC), the posterior intraparietal sulcus (pIPS), the supplementary motor area (SMA), the anterior cingulate cortex (ACC), the lateral prefrontal cortex (IPFC), and the anterior insula. Subcortical activations were identified in the thalamus, caudate nucleus and cerebellum. 

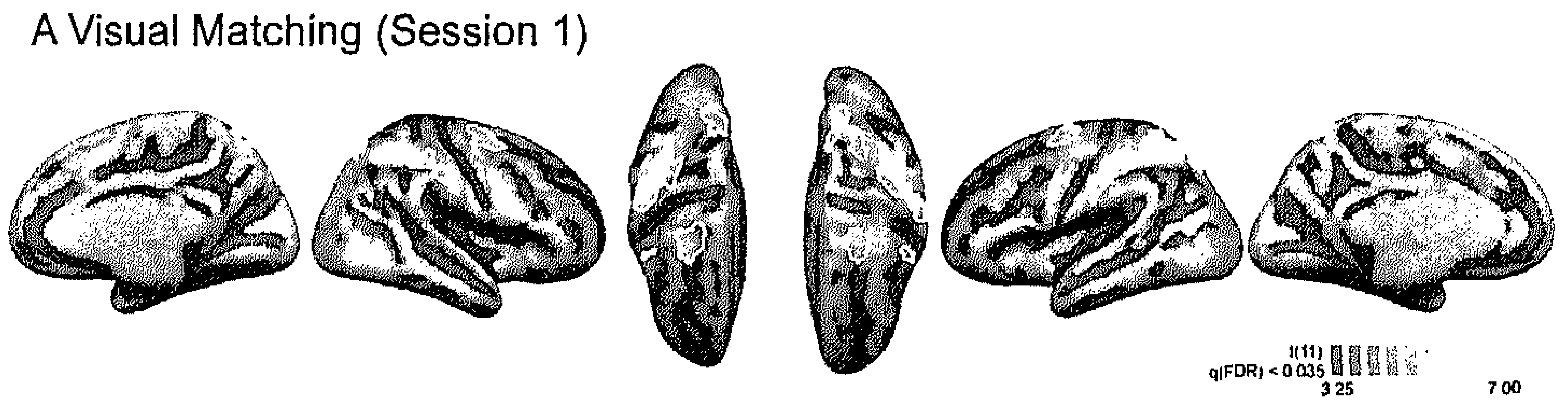

B Visual Matching (Session 2)

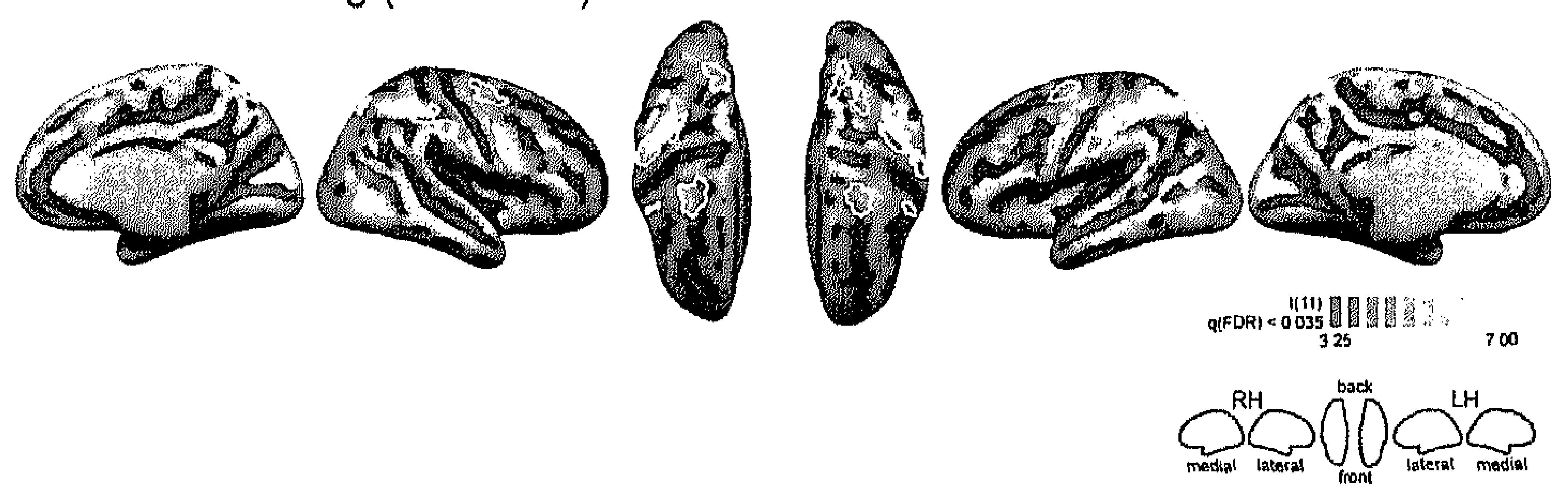

Figure S2. Group activation maps showing regions implicated in the visual matching task (A) Activation map showing the brain areas which were activated in all three visual matching conditions in scanning session 1 (based on a conjunction analysis against baseline), projected on the inflated average group cortex mesh (random effects; $t(11)=3.25 ; \mathrm{q}(\mathrm{FDR})<0.035$ ). Note that all of the learning-related areas which were identified during the actual execution of the movement sequences (marked by white contours) are now reactivated again (as part of a more extended network), although subjects are not engaged in any physical motion. (B) When performing the same contrast on the visual matching task data collected four days later in the second scanning session, a remarkably similar activation pattern was observed, suggesting that the task was performed in a similar manner on both occasions. Abbreviations: $\mathrm{RH}$, right hemisphere; $\mathrm{LH}$, left hemisphere.

\section{Supplemental Discussion}

From a critical point of view, one might argue that the observed activation patterns in the current study were caused by differences in the global attention level across conditions. Because the non-matching trajectories started to diverge from the performed sequences at one point or another, subjects might have been tempted to no longer pay attention to the presented stimuli once this first divergence occurred (as they could be sure of what to respond at the end of the trial, without requiring any further information). This would result in a lower activation level when compared to the matching stimuli which needed to be attended until completion if a match had to be reliably established. There are two arguments which can be raised against this alternative explanation. First, if a genetal 'de-allocation' of attention would take place, such an effect would be 
expected throughout a wider set of regions. However, no differential response patterns were observed in for example the LOC (Figure 3D), a region which is not implicated in motor learning but whose responses are known to be strongly modulated by attention (e.g., [S1]). Secondly, the divergent results with respect to the two matching stimulus types seem incompatible with such an account. If the described effect would be caused by a general attentional effect, the responses to both matching trajectories should be enhanced compared to the non-match trials. However, this was not the case in the majority of the tested Regions of Interest (ROIs). In addition, if the matching trajectories would be expected to evoke different responses compared to the non-match events based on such arguments, one would expect that the not extensively trained 'OLD' sequence would require more attention to be correctly identified and therefore cause stronger (attentionally enhanced) responses (a response profile which actually was observed for the right ACC). This however is not supported by the presented data. On the contrary, the visual representation of the overlearned sequence gave rise to the strongest responses in the identified ROIs, suggesting that the response amplitude was more strongly linked to the level of skill consolidation than to the amount of allocated attentional resources.

\section{Supplemental Experimental Procedures}

\section{Motor Learning Task}

During the motor learning part of the first scanning session, subjects learned two novel movement sequences by tracing predefined maze trajectories while keeping their eyes closed. Both mazes were matched with respect to their basic characteristics: they had the same total length of $260 \mathrm{~mm}$, consisted of eight segments which were connected by right-angled corners and included a 'deadend' at each intersection (see Figure 1A). Furthermore, the straight-line segments constituted a closed loop, so that the corresponding movement sequence could be continuously repeated. Because no visual feedback on the required movements was provided throughout the experiment, subjects had to online process the somatosensory and proprioceptive cues they could extract while performing the movement sequences, and therefore had to literally 'learn by doing'. Their instructions were to continuously trace the maze path as fast and as accurately as possible, and to complete the sequences in a clockwise fashion. Because learning to execute the required sequences would result in faster performance, a simple square trajectory was traced either in a fast or slow fashion in two separate control conditions to be able to distinguish performancerelated from true learning-related changes in neural activity [S2]. Tracing was performed in twelve continuous blocks of $30 \mathrm{~s}$, interleaved with resting periods 
lasting $20 \mathrm{~s}$. The mazes were fixed to a specifically designed table covering the subjects' abdomen, and the required movements mainly had to be performed by moving the wrist joint of the right hand. The arm, shoulder and head were fixated with foam padding to minimize task-related motion. The pen tracing movements were recorded using a high-resolution and MR-compatible resistance-based recording method [S3].

During the following three days, subjects extensively trained one of the sequences they started to learn in the first scanning session. Therefore, when being re-scanned four days later, a comparison between the extensively trained and the only briefly practiced sequence could be performed. The order in which the different tracing conditions were presented and the sequence which was extensively trained were counter-balanced across subjects.

\section{Visual Matching Task}

In both scanning sessions, the motor learning task and the visual matching task were part of the same functional run. After signaling to the subject that the eyes could be opened, a short introductory text was presented as a reminder on the task instructions. All images were projected onto a frosted screen (which was positioned at the rear end of the MR scanner bore and could be viewed via a mirror mounted onto the head coil) using a liquid crystal display (LCD) projector (PLC-XT11-16, Sanyo North America Corporation, San Diego, USA). The presented stimuli were generated with the 'Presentation' software package (v9.2, Neurobehavioral Systems, San Francisco, USA). 'Match' and 'nonMatch' decisions were recorded via button presses with the right index or middle finger, respectively (LUMItouch keypads, Photon Control, Burnaby, Canada). Because no visual feedback was provided throughout the entire session, subjects most likely reverted to the information they were able to retrieve on the motor aspects of the learned movements. Importantly, the white rectangle did not leave a trace (rendering the matching to a holistic visual template of the movement almost impossible), which presumably led subjects to rely even more on the stored motor representation of the performed routines to guide their judgment. All displayed trajectories had the same total length, consisted of eight straight-line segments which were interconnected by $90^{\circ}$ angles, and started with a vertical segment represented by an upward movement. The starting point of each trajectory was slightly jittered across trials to prevent any regularity between the type of stimulus and its starting position, which might have made the task trivial once discovered. The white rectangle moved at a constant velocity (roughly matching the velocity at which the sequences were actually executed before), always reaching its starting position again after three imaging volumes were acquired (corresponding to $3750 \mathrm{~ms}$ ). 
The inter-stimulus interval was either $10,12.5$ or $15 \mathrm{~s}$. A total of 20 matching and 20 non-matching trajectories were randomly presented.

During the additional button press control (BPC) task which was performed at the end of the second scanning session, a white rectangle was displayed either right or left from the fixation cross for $1250 \mathrm{~ms}$ in a total of 16 trials presented in a slow event-related fashion similar to the main task. Subjects were instructed to press the button corresponding to the location of the presented rectangle (again, only the right index and middle finger were used). The control task was not intermixed with the experimental task to prevent any ambiguity with respect to the trial type. The order in which the experimental and control task were presented was not balanced across subjects to ensure that the time between the actual execution of the sequences and the visual matching task was similar for all subjects.

\section{Image Acquisition}

Images were acquired on a 3T Siemens Magnetom Allegra head-scanner (Siemens Medical Systems, Erlangen, Germany), using a standard volume coil. Anatomical 3D datasets consisting of 176 slices were collected at the beginning of each scanning session (MDEFT sequence [S4]; Repetition Time (TR) $=7.92$ $\mathrm{ms}$; Echo Time $(\mathrm{TE})=2.4 \mathrm{~ms}$; Flip Angle $=15^{\circ}$, voxel dimensions $=1 \times 1 \times 1$ $\mathrm{mm}^{3}$; Field of View $(\mathrm{FoV})=256 \times 224 \mathrm{~mm}^{2}$ ). The subsequent functional measurements were all collected within a single continuous run, to minimize scanner-related changes in the overall signal level across the different conditions and tasks. A gradient-echo echo-planar imaging sequence was used to record 2116 functional volumes in the first, and 2244 functional volumes in the second scanning session, covering the whole brain by including 21 transversal slices $\left(\mathrm{TR}=1250 \mathrm{~ms}\right.$; $\mathrm{TE}=28 \mathrm{~ms}$; Flip Angle $=67^{\circ}$; voxel dimensions $=3.5 \times 3.5 \times 5 \mathrm{~mm}^{3}$; gap $=1 \mathrm{~mm} ; \mathrm{FoV}=224 \times 224 \mathrm{~mm}^{2}$ ).

\section{Data Analysis}

Preprocessing and analyses of the acquired imaging data were performed using the routines implemented in BrainVoyager QX (v1.7; Brain Innovation, Maastricht, The Netherlands). After correcting for intensity inhomogeneities, the anatomical datasets from both scanning sessions were aligned to each other and transformed into Talairach space [S5]. Next, all transformations were reapplied in a single step, and both datasets were averaged. In addition, a semiautomatic segmentation of the grey/white matter boundary was performed [S6]. Based on the individual brains' curvature information, a cortex-based intersubject alignment was run, applying a 'moving target' group averaging 
approach [S7]. Finally, using the information from the alignment procedure, a folded average cortex representation was constructed, in order to present the data in a space which on average suits the measured subject sample best.

Although the data from the motor learning and the visual matching task were part of the same functional run, they were separately analyzed to allow the use of optimized preprocessing settings as well as to independently estimate the resting baseline for conditions in which subjects had their eyes closed (motor learning task) or open (matching task). Only the analysis steps with respect to the visual matching task data will be described here. After slice scan time correction, 3D motion correction was performed by aligning all acquired volumes to a single volume in the middle of the motor learning part of the first scanning session via rigid-body transformations. Temporal filtering included linear trend removal and high-pass filtering at 13 (session 1) or 18 (session 2) cycles per timecourse (corresponding to 0.019 and $0.020 \mathrm{~Hz}$, respectively). No spatial smoothing was performed. Finally, the functional data were interpolated to a $3 \times 3 \times 3 \mathrm{~mm}^{3}$ voxel resolution, and were sampled along the individuals' white/grey matter boundary to allow the alignment of functional data sets across subjects based on the aforementioned cortex-based alignment procedure. Activation maps were obtained by applying a standard voxel-wise General Linear Model (GLM) at the single subject level. The 3D motion parameters (as estimated during preprocessing) were included in the design matrices, together with two predictors representing a correctly identified match (1 for each sequence), a predictor for correctly identified non-matches, and finally a compound predictor to describe all incorrect or omitted responses. For the second scanning session, two additional predictors were included, representing the two types of button presses from the control condition. To directly compare the matching and the button press control task, an additional GLM was run in which equal event durations were specified for all included conditions. This approach was chosen because the required button presses immediately followed the events we were mainly interested in and therefore would be hard to model in isolation. By specifying time-intervals with equal durations which included the required button presses for both tasks, we at least were able to assure that the identified reactivations of the motor learning regions in the visual matching task were not solely related to the performed button presses, even though the contrasted conditions were sub-optimally balanced with respect to ongoing visual stimulation. All boxcar predictors were convolved with a theoretical Two Gamma hemodynamic response function. The reported results focused on the outcomes at the group level, obtained by random effects analyses. Unless stated otherwise, statistical maps on the group level were thresholded at $t=3.25$ with a corresponding probability of $\mathrm{p}$ (uncorr) $<0.008$. Additional random effects analyses were carried out at the level of single ROIs, which were functionally defined in the motor learning part of the first scanning session. 


\section{Supplemental References}

S1. Murray, S.O., \& Wojciulik, E. (2004). Attention increases neural selectivity in the human lateral occipital complex. Nature Neuroscience, 7, 70-74.

S2. van Mier, H., Tempel, L.W., Perlmutter, J.S., Raichle, M.E., \& Petersen, S.E. (1998). Changes in brain activity during motor learning measured with PET: effects of hand of performance and practice. Journal of Neurophysiology, 80, 2177-2199.

S3. Reithler, J., Reithler, H., van den Boogert, E., Goebel, R., \& van Mier, H. (2006). Resistance-based high resolution recording of predefined 2-dimensional pen trajectories in an AMRI setting. Journal of Neuroscience Methods, 152, 10-17.

S4. Deichmann, R., Schwarzbauer, C., \& Turner, R. (2004). Optimisation of the 3D MDEFT sequence for anatomical brain imaging: technical implications at 1.5 and $3 \mathrm{~T}$. Neurolmage, 21, 757-767.

S5. Talairach, J. \& Tournoux, P. (1988). Co-planar stereotaxic atlas of the human brain, (Stuttgart: G. Thieme).

S6. Kriegeskorte, N., \& Goebel, R. (2001). An efficient algorithm for topologically correct segmentation of the cortical sheet in anatomical MR volumes. NeuroImage, 14, 329-346.

S7. Goebel, R., Esposito, F., \& Formisano, E. (2006). Analysis of functional image analysis contest (FIAC) data with BrainVoyager QX: From single-subject to cortically aligned group General Linear Model analysis and self-organizing group Independent Component Analysis. Human Brain Mapping, 27, 392-401. 


\section{Chapter 5:}

General discussion 
The work presented in the current thesis focused on the neural representations of continuous motor sequences. One the one hand, the way these representations might change throughout the course of learning was investigated. On the other hand, the processes involved in accessing information on stored movement sequences were probed in the context of an action observation paradigm in which purely visual representations of the previously learned (yet unseen) actions were used. In this chapter, the results of the described studies will be discussed within a broader framework.

One of the main characteristics of the motor learning task employed in the conducted experiments was that it involved continuous pen tracing movements, instead of discrete and relatively simple button presses as used in the vast majority of neuroimaging studies on sequence learning. In addition, the required movements had to be performed in the absence of any visual guidance, allowing to more directly focus on motor aspects of learning per se (i.e., in contrast to visuo-motor processing during which the specific contributions of visual and motor components might be harder to disentangle). Consequently, a novel setup needed to be developed which allowed the recording of the subjects' pen tracing movements in an $\mathrm{MRI}$ environment. In addition, in-house software needed to be written to efficiently acquire, pre-process and analyze the pen tracing data. As described in Chapter 2, the method which was ultimately used to allow such detailed behavioral recordings was based on a measurement scheme in which the traversed path was reconstructed from multi-facetted resistance measurements. Following extensive testing and fine-tuning, the calibration and validation results unequivocally established that robust and sufficiently detailed behavioral data could be obtained while simultaneously collecting functional brain images. The applied method was shown to adequately handle adverse fluctuations in the contact resistance between the stylus and the maze path, turned out to provide sufficient flexibility and was proven to be fully MRcompatible.

Even though the developed setup met all necessary requirements for the current application, several useful extensions are conceivable. Foremost, it might be possible to adapt the introduced recording method in such a way that general unrestricted pen tracing movements in two dimensions could be supported. This would for example allow the measurement of brain activations during free recall conditions (in the absence of a predefined maze path), or the inclusion of learning conditions exclusively based on information provided through other modalities (e.g., verbal descriptions, visual cues). Although alternative solutions to record less restricted, two dimensional stylus-based movement kinematics in an fMRI setting have recently been proposed (Hauptmann et al., 2004; Zakzanis et al., 2005), no dedicated reports on these alternative methods have been published to date, hampering any quantitative comparisons with respect to the 
Chapter 5

spatial and temporal resolution each of the applied methods can offer. Secondly, an interesting possibility to extend the setup's functionality would be to implement the pre-processing and analysis of the recorded tracing movements in real-time. Such a more versatile implementation would allow the application of more flexible imaging protocols. For example, by monitoring the subject's performance in real time, an event-related fMRI design could be used in which each completed loop along the maze path could function as an isolated event. By analyzing the tracing movements online, the completion of each loop could be (auditorily) signaled to the subject. This information would be crucial during the early learning stages, because it would otherwise be impossible for the subjects to determine whether the initial starting position was reached again (marking the completion of a loop), as they would not yet be familiar with the maze's geometry. Similarly, instead of generally linking the amount of practice to the time spend on the task, behavioral criteria related to the subject's level of proficiency could be used to individually adjust the scanning protocol in order to equalize the achieved progress across subjects in terms of performance.

The introduced resistance-based tracing setup was employed in the AMRI study on continuous motor sequence learning described in Chapter 3. Mainly based on the observation that relatively few studies have taken into account extended practice conditions (Doyon, Penhune, \& Ungerleider, 2003), and further motivated by the largely diverging results reported on practice-induced neurovascular activation changes (van Mier, 2000; Kelly \& Garavan, 2005), this experiment was designed to investigate the effects of learning at two different time scales. By including two different scanning sessions separated by several days of extensive training, gains in performance levels as well as changes in neural activation patterns could be closely monitored throughout the course of learning.

The first important step in the analysis of the neuroimaging data was the identification of areas showing learning-related response profiles. Based on a contrast between the maze tracing conditions and the appropriate control conditions, a strongly symmetrical bilateral network of cortical regions was demonstrated to be implicated in learning the novel movement sequences. Their involvement was shown to be independent of any increases in movement velocity or general motor output which could be expected to accompany the transition to more advanced levels of proficiency at executing the required tracing sequences. Next, the ongoing involvement of the identified Regions of Interest (ROIs) was examined by contrasting the initial stage of learning with a similar time interval at the end of the learning period. The exact intervals under investigation were selected at the single subject level, based on the concurrently measured behavioral performance. Interestingly, changes in activation strength could already be observed within the first scanning session, after a few minutes 
of training. The revealed inverse relationship between activation levels and behavioral improvements was generally restricted to the defined learningrelated ROIs, further underlining their role in skill acquisition. This finding was nicely replicated in the second scanning session (i.e., four days later), both when a completely novel maze had to be learned and when training continued on a previously encountered, yet not extensively trained maze. Importantly, no such additional learning-related activation decreases were found when subjects performed the overlearned sequence, which they had executed hundreds of times on the days in between the two AMRI sessions.

These results have a number of implications. On a practical level, they show that defining a 'learning baseline' to which more advanced levels of task execution can be contrasted is not a trivial matter. Even though pooling the successive tracing trials of the first scanning session was an appropriate strategy in terms of detection power when identifying the brain areas associated with learning, contrasting the early and late learning phases within the first scanning session already revealed decreases in activation levels during learning. This implies that it is worthwhile to directly examine the dynamic nature of the process under investigation right from the start, instead of assuming to have established a valid static baseline for further comparisons by simply averaging the data collected within an initial fMRI measurement session. And although incorporating a subject-specific early $v s$. late contrast can already be seen as an improvement in this respect, slightly more sophisticated approaches might have rendered the analyses even more sensitive to the ongoing changes in neural representation. For example, one might imagine that instead of using the two extremes of the performance continuum (early $v s$. late as defined by the trials at which the subject's performance was between $0-25$ and $75-100 \%$ of his or her maximum performance), the complete subject-specific behavioral performance time course could be used as a predictor during the analysis of the brain's responses in the future. Additionally, one might incorporate specific performance variables (i.e., the occurrence of tracing errors or stops) as separate predictors in order to isolate the associated transient activations which are now potentially missed by analyzing the data in a blocked fashion (although of course, one potential problem one faces here is that these additional behavioral variables are probably highly correlated with the tracing velocity, which was used as the principal parameter to describe the subjects' learning curve). Similarly, it might be interesting to try and capture the involvement of different brain regions within a single 30 seconds trial. Because subjects repeatedly performed the same movement sequence within a given trial, it is conceivable that some areas were only relevant during the first few loops through the maze in order to recall the appropriate movement sequence from memory and verify its correct re-enactment by other neural structures. Again, such transient activations might have been too weak to be detected by the analysis approach 
chosen in the reported studies (see also Duff et al., 2007). Nevertheless, the activation patterns which were readily observable by the performed analyses provided several interesting insights. Besides showing that a widespread network of bilaterally activated brain areas was implicated in learning the novel movement sequences, the findings also established that the basic constellation of this learning-related network was rather stable throughout the entire learning process. This suggests that learning is not necessarily related to dramatic functional reorganizations at the neural systems level, in the sense that completely different regions come into play depending on the learning phase.

Interestingly, Kelly \& Garavan (2005) have recently presented a classification scheme in which they attempted to categorize the different practice-induced changes in neural activation patterns reported in the neuroimaging literature. In this scheme, one possible outcome of practice was described as a 'redistribution' of functional activations, in which the relative contribution of the involved areas changes with practice, although the general layout of the implicated network remains unchanged (in contrast to a real 'reorganization' of activations in which different areas are recruited depending on the stage of learning). At first glance, the results from the motor learning study described above seem to be in accordance with such a functional redistribution. However, a closer look suggests that the data do not completely match the proposed definition. For example, an important criterion put forward for an emergent redistribution due to practice is the absence of activation in so-called scaffolding attentional/control areas during automatic performance. Even though one of the areas in which we found strong activation decreases with practice, namely the posterior parietal cortex (PPC), is regularly seen as one of these domain-general control regions (e.g., Chein \& Schneider, 2005), this might not be sufficient to characterize the neural correlate of the observed learning process in terms of a redistribution of functional activations. Instead, the role of the PPC might be specific for the task at hand, for example related to the generation of abstract motor intentions or the construction of a template of the traced trajectory through visuomotor imagery. Although this task-specific function of the PPC might become less pronounced during later stages of learning, the PPC does seem to show an enhanced activation at the beginning of each trial, even during automatic execution. The fact that this more transient response is not detected as a significant activation is more indicative of the lack of detail which can be captured by the used analysis model than the role of the PPC itself (and underlines the importance of inspecting the time courses which can be extracted for each given ROI). Together with the complete lack of involvement of other typical scaffolding regions (such as the prefrontal and anterior cingulate cortex) throughout the entire course of learning, and the observation that not a single ROI showed enhanced response amplitudes when contrasting the late $v s$. early stages of learning, the continued involvement of 
the PPC seems to suggest that we can not speak of a true redistribution of functional activations for the current tracing task according to the criteria listed by Kelly \& Garavan (2005). In contrast, the reported results seem more in line with a neural implementation of learning in which a more proficient performance level is linked to more efficient processing within a consistently recruited network of cortical regions. Even though significant decreases in activation could be observed in the identified ROIs, there was a substantial overlap in activation patterns across sessions and levels of proficiency, in accordance with recent results from other experiments in which motor learning was studied across multiple scanning sessions (de Weerd et al., 2003; Wu et al., 2004; Poldrack et al., 2005), as well as studies in which the neural activation patterns of novices were compared to those of experts (Haslinger et al., 2004; Meister et al., 2005).

However, this representation still is in need of some refinement. Even though none of the identified learning-related ROIs showed enhanced activation levels accompanying improvements in performance, the posterior section of the left putamen, which is part of the subcortical basal ganglia system, did show increased activation levels when the overlearned compared to the novel sequence needed to be executed in the second scanning session. Interestingly, Doyon and colleagues have recently proposed a model describing the contribution of cortico-striatal and cortico-cerebellar networks during several stages of skill acquisition (Doyon \& Ungerleider, 2002; Doyon, Penhune, \& Ungerleider, 2003; Doyon \& Benali, 2005). In their view, both these systems are initially recruited when learning a new movement sequence, whereas only the cortico-striatal network remains active in later stages due to its role in the consolidation and long-term storage of the acquired motor routine. Our results are only partially consistent with this hypothesis. Although cerebellar and striatal activations were found next to several cortical activations, these subcortical structures did not seem to be specifically involved in learning, and their involvement was generally not restricted to a particular time window. On the other hand, the enhanced posterior putamen responses while tracing the extensively trained maze path are in agreement with the cited model, and suggest that this structure is engaged in the re-instatement of previously learned movement sets (Miyachi et al., 2002; Lehericy et al., 2005). However, because the main focus of the current study was to investigate the changes in representation that occur at the neural systems level throughout the time course of learning, determining the exact role of each of the identified network's nodes still awaits further research. In addition, an alternative way in which learning mechanisms could be expressed might involve changes in the connectivity between the involved brain regions, forming a potentially fruitful line of research which should more actively be pursued (Doyon \& Benali, 2005). 
The study described in Chapter 4 was designed to answer the following questions: 1) will the same brain regions that were involved in the nonvisual learning of a set of motor sequences be recruited when information on the stored motor routines needs to be retrieved based on an abstract visual representation of the traversed trajectories? 2) if so, will these brain areas display graded responses in the sense that the observed level of activation is dependent on how familiar subjects are with executing a particular movement sequence? In the reported experiment, subjects were confronted with a visual representation of the movement sequences they previously learned to perform in the absence of any visual cues. In the context of a forced-choice matching task, they were instructed to indicate whether the presented trajectories matched one of the learned movement sequences or not. The obtained results revealed that a distributed network of bilateral premotor and parietal areas, which had earlier shown activation changes specifically related to learning the required movement sequences, was now reactivated during the perceptual matching task. This finding nicely corroborates recent reports on the existence of a human equivalent of the 'mirror neuron' system as first described in the monkey (Rizzolatti \& Craighero, 2004). This mirror neuron system is thought to play a pivotal role in 'action understanding', as put forward in the so-called 'direct matching' hypothesis (Rizzolatti, Fogassi, \& Gallese, 2001). This hypothesis states that the observation of an action activates corresponding motor representations in neural circuits of the observer's brain, which are also engaged in the actual execution of the same action when performed by the observer himself. Because the outcome and the meaning of the observed action become evident by this internal simulation, the intentions of the observed person can ultimately be inferred. This direct matching of sensory input to covertly reenacted motor responses would therefore allow what has been described as the transfer from "visual information to knowledge" (Rizzolatti \& Craighero, 2004, p. 172). In accordance with earlier neurophysiological work in the monkey, the human ventral premotor area (PMv; encompassing both Brodmann's area (BA) 44and the inferior part of BA 6), and the anterior part of the inferior parietal lobule have been assigned a prominent role within this system (Buccino et al., 2001; Binkofski \& Buccino, 2006). These areas are generally considered as the human homologues of monkey areas $\mathrm{F} 5, \mathrm{~F} 4$, and $\mathrm{PF} / \mathrm{PFG}$, respectively (following the nomenclature introduced by Matelli, Luppino, \& Rizzolatti, 1985). The present study, however, supports the results from a number of recent fMRI studies showing that similar mirror responses can be found in a larger set of regions for which no single cell recording data regarding potential mirror properties have been collected to date (e.g., Frey \& Gerry, 2006; Gazzola et al., 2007). In addition, the current findings suggest that the human mirror system is not strictly tuned to so-called biological motion. In other words, the observed 
actions do not necessarily have to be performed by another human being in order to elicit a mirroring response in the brain of the observer (see also Press et al., 2005). What is interesting in this respect, is that we indeed did not find any activation in the posterior superior temporal sulcus (pSTS), which has been characterized as a source of information for the mirror neuron areas (Iacoboni \& Dapretto, 2006), and is believed to be selective for stimuli portraying biological agents (Allison, Puce \& McCarthy, 2000). Furthermore, the current findings are in agreement with recent neuroimaging data which suggest that the human mirror neuron system might be more broadly tuned in general. For example, several areas in the human brain (both within and outside the network of areas which constitute the putative human equivalent of the monkey mirror neuron system) apparently do react to mimicked movement (Grezes et al., 2003), both when observing object-oriented (Buccino et al., 2001) as well as intransitive actions (Sakreida et al., 2005), whereas no such responses to mimicked actions were observed using single cell recordings in the monkey (e.g., Umilta et al., 2001). In sum, mirror responses in humans can be found in a more widespread network of task-specific cortical areas than included in the classically defined mirror neuron system, and relatively abstract visual stimuli can be sufficient to trigger the corresponding motor representations in the observer's brain.

Another important point related to the functionality of the mirror neuron system was addressed by examining whether the implicated regions would not only be recruited when trying to match the presented visual input to an already available motor representation, but would additionally respond more vigorously when such a one to one match could actually be established. Evidence that this would be the case came from an action observation study by Buccino and colleagues (2004) in which the brain's responses while observing actions performed by other humans and non-conspecifics (e.g., monkeys, dogs) were contrasted. The results suggested that the extent to which a given stimulus succeeds in activating the resonance system depends on the existing motor repertoire of the observer. Or, put differently, the level of engagement of the mirror neuron system is determined by whether the observer is capable to produce the observed movement at all, and if so, the level of experience the observer has with executing the particular action. Similarly, two other recent $\mathrm{AMRI}$ studies have pointed in the same direction. Calvo-Merino et al. (2005) reasoned that if action observation causes the observer to internally simulate the observed action, the related brain processes would be modulated by the expertise of the observer. To test this hypothesis, they let three groups of subjects (dance experts in one of two styles and a control group) watch videos in which stylespecific dance sequences were performed. Their findings showed that the activated brain regions showed stronger responses in both expert groups when the observed movements were part of their own area of expertise, while the control group responded equally to movements belonging to either dance style. 
Likewise, Cross et al. (2006) contrasted observing trained versus unpracticed dance sequences over a training period of five weeks in a within-subjects design. Besides replicating the contribution of a distributed network of areas in action simulation and observation, they showed that the activity in the inferior parietal lobule and the ventral premotor cortex was modulated by the dancers' experience and ratings of their own ability to perform the observed movements. Consistent with these reports, the results from our own fMRI experiment show that the previously defined motor learning ROIs displayed differentiated responses during the visual matching task. On the one hand, the visually presented trajectories which matched the previously executed (but unseen) movements elicited stronger responses compared to the non-matching trajectories. On the other hand, a consistently observable trend was revealed when looking at the two matching trajectory types. Generally, the involved brain regions tended to respond more strongly when perceiving the trajectory which corresponded to the overlearned movement sequence, whereas a previously encountered, but not extensively trained movement trajectory caused slightly weaker responses. However, in contrast to both dance studies mentioned previously, the current study included the appropriate motor learning conditions to actually establish that the same brain areas were involved while learning to execute the corresponding movements, and ruled out the possibility that the stronger responses could be explained by a purely perceptual effect, namely a more extensive visual familiarity for the well-learned action, by only incorporating nonvisual motor learning conditions (see also Calvo-Merino et al., 2006).

Although the exploration of the mirror neuron system's properties represents a very active line of research, there still are several issues in need of further clarification. One interesting aspect in this regard is that recent neuroimaging findings from fMRI experiments in humans could currently constitute the basis for further electrophysiological research in non-human primates (in which mirror neurons were first discovered). For example, as mentioned above, several fMRI studies have shown that a widespread network of cortical regions beyond the classically defined mirror neuron system displays response profiles which are in line with the defining criteria used to characterize mirror neuron responses. These results provide additional regions of interest which should be examined more closely when trying to understand the mirror neuron system's workings in more detail. Similarly, several IMRI studies have shown that the human mirror neuron system strongly responds to video clips of recorded motor acts (e.g., Molnar-Szakacs et al., 2006; Gazzola et al, 2007), whereas earlier electrophysiological work suggested that a resonance response could only be evoked by a physically present actor. Probably inspired by these findings in humans, reports on similar responses to videotaped actions are now emerging based on single cell recordings in the monkey (Caggiano et al., 2007). There are 
also a number of additional conceptual issues which remain to be solved independent of the used research method. These include the question whether mirror neurons predominantly code the end-goal of an action (e.g., Hamilton \& Grafton, 2006) or whether (subsets of) these neurons also form representations of the means by which this end-goal was achieved (e.g., Borroni et al., 2005; Filimon et al., in press). Likewise, a debate is currently centering on the functional role of the recorded mirror neuron responses. Are they mainly implicated in the understanding (e.g., Fogassi et al., 2005; Iacoboni et al., 2005) or the prediction (Prinz, 2006; Schubotz, 2007) of observed actions? And how can these responses be linked to the proposed forward models the brain seems to use during action generation (Miall, 2003)? Moreover, it would be interesting to know more about the mechanisms which somehow prevent that internal simulations of observed movements do not more often lead to involuntary overt imitations of what an observer sees (Brass \& Heyes, 2005). Finally, it seems worthwhile to investigate whether all these gained insights could eventually play a role in the development of promising neurorehabilitation strategies (e.g., Buccino, Solodkin, \& Small, 2006; Ertelt et al., 2007). 


\section{Chapter 5}

\section{References}

Borroni, P., Montagna, M., Cerri, G., \& Baldissera, F. (2005). Cyclic time course of motor excitability modulation during the observation of a cyclic hand movement. Brain Research, 1065, 115-124.

Brass, M., \& Heyes, C. (2005). Imitation: Is cognitive neuroscience solving the correspondence problem? Trends in Cognitive Sciences, 9, 489-495.

Buccino, G., Binkofski, F., Fink, G.R., Fadiga, L., Fogassi, L., Gallese, V., Seitz, R.J., Zilles, K., Rizzolatti, G., \& Freund, H-J. (2001). Action observation activates premotor and parietal areas in a somatotopic manner: an fMRI study. European Journal of Neuroscience, 13, 400-404.

Buccino, G., Lui, F., Canessa, N., Patteri, I., Lagravinese, G., Benuzzi, F., Porro, C.A., \& Rizzolatti, G. (2004). Neural circuits involved in the recognition of actions performed by non-conspecifics: an fMRI study. Journal of Cognitive Neuroscience, 16, 1-14.

Buccino, G., Solodkin, A., \& Small, S.L. (2006). Functions of the mirror neuron system: Implications for neurorehabilitation. Cognitive and Behavioral Neurology, 19, 55-63.

Binkosfki, F., \& Buccino, G. (2006). The role of the ventral premotor cortex in action execution and action understanding. Journal of Physiology - Paris, 99, 396-405.

Caggiano, V., Fogassi, L., Giese, M., Rizzolatti, G., Thier, P., \& Casile, A. (2007). Mirror neurons responding to filmed actions. $37^{\text {th }}$ annual meeting of the Society for Neuroscience, San Diego, USA.

Calvo-Merino, B., Glaser, D.E., Grezes, J., Passingham, R.E., \& Haggard, P. (2005). Action observation and acquired motor skills: An fMRI study with expert dancers. Cerebral Cortex, 15, 1243-1249.

Calvo-Merino, B., Grezes, J., Glaser, D.E., Passingham, R.E., \& Haggard, P. (2006). Seeing or doing? Influence of visual and motor familiarity in action observation. Current Biology, 16, 1905-1910.

Chein, J., \& Schneider, W. (2005). Neuroimaging studies of practice-related change: fMRI and meta-analytic evidence of a domain-general control network for learning. Cognitive Brain Research, 25, 607-623.

Cross, E.S., Hamilton, A.F.C., \& Grafton, S.T. (2006). Building a motor simulation de novo: Observation of dance by dancers. NeuroImage, 31, 1257-1267.

de Weerd, P., Reinke, K., Ryan, L., McIsaac, T., Perschler, P., Schnyer, D., Trouard, T., \& Gmitro, A. (2003). Cortical mechanisms for acquisition and performance of bimanual motor sequences. Neurolmage, 19, 1405-1416. 
Doyon, J., \& Ungerleider, L.G. (2002). Functional anatomy of motor skill learning. In: L.R. Squire, \& D.L. Schacter (Eds.), Neuropsychology of memory (pp. 225-238), New York (NY): Guilford Press.

Doyon, J., Penhune, V., \& Ungerleider, L.G. (2003). Distinct contribution of the cortico-striatal and cortico-cerebellar systems to motor skill learning. Neuropsychologia, 41, 252-262.

Doyon, J., \& Benali, H. (2005) Reorganization and plasticity in the adult brain during learning of motor skills. Current Opinion in Neurobiology, 15, 161-167.

Duff, E., Xiong, J., Wang, B., Cunnington, R., Fox, P., \& Egan, G. (2007). Complex spatio-temporal dynamics of fMRI BOLD: A study of motor learning. Neurolmage, 34, 156-168.

Ertelt, D., Small, S., Solodkin, A., Dettmers, C., McNamara, A., Binkosfki, F., \& Buccino, G. (2007). Action observation has a positive impact on rehabilitation of motor deficits after stroke. Neurolmage, 36, 164-173.

Filimon, F., Nelson, J.D., Hagler, D.J., \& Sereno, M.L. (in press). Human cortical representations for reaching: Mirror neurons for execution, observation, and imagery. NeuroImage.

Fogassi, L., Ferrari, P.F., Gesierich, B., Rozzi, S., Chersi, F., \& Rizzolatti, G. (2005). Parietal lobe: from action organization to intention understanding. Science, 308, 662667.

Frey, S.H., \& Gerry, V.E. (2006). Modulation of neural activity during observational learning of actions and their sequential orders. Journal of Neuroscience, 26, 1319413201 .

Gazzola, V., Rizzolatti, G., Wicker, B., \& Keysers, C. (2007). The anthropomorphic brain: The mirror neuron system responds to human and robotic actions. Neurolmage, $35,1674-1684$.

Grezes, J., Armony, J.L., Rowe, J., \& Passingham, R.E. (2003). Activations related to "mirror" and "canonical" neurons in the human brain: an AMRI study. NeuroImage, 18, 928-937.

Hamilton, A.F., \& Grafton, S.T. (2006). Goal representation in human anterior intraparietal sulcus. Journal of Neuroscience, 26, 1133-1137.

Haslinger, B., Erhard, P., Altenmueller, E., Hennenlotter, A., Schwaiger, M., Graefin von Einsiedel, H., Rummeny, E., Conrad, B., \& Ceballos-Baumann, A. (2004). 


\section{Chapter 5}

Reduced recruitment of motor association areas during bimanual coordination in concert pianists. Human Brain Mapping, 22, 206-215.

Hauptmann, B., Sosnik, R., Smikt, O., Okon, E., Flash, T., \& Karni, A. (2004). A new method to control for $2 \mathrm{D}$-movement kinematics during functional magnetic resonance imaging (fMRI). Program No. 533.18. 2004 Abstract Viewer/Itinerary Planner. Washington, DC: Society for Neuroscience.

Iacoboni, M., Molnar-Szakacs, I., Gallese, V., Buccino, G., Mazziotta, J.C., \& Rizzolatti, G. (2005). Grasping the intentions of others with one's own mirror neuron system. PLoS Biology, 3, e79.

Iacoboni, M., \& Dapretto, M. (2006). The mirror neuron system and the consequences of its dysfunction. Nature Reviews Neuroscience, 7, 942-951.

Kelly, A.M.C., \& Garavan, H. (2005) Human functional neuroimaging of brain changes associated with practice. Cerebral Cortex, 15, 1089-1102.

Lehericy, S., Benali, H., van de Moortele, P-F., Pelegrini-Issac, M., Waechter, T., Ugurbil, K., \& Doyon, J. (2005). Distinct basal ganglia territories are engaged in early and advanced motor sequence learning. Proceedings of the National Academy of Sciences USA, 102, 12566-12571.

Matelli, M., Luppino, G., \& Rizzolatti, G. (1985) Patterns of cytochrome oxidase activity in the frontal agranular cortex of the macaque monkey. Behavioral Brain Research, 18, 125-136.

Meister, I., Krings, T., Foltys, H., Boroojerdi, B., Mueller, M., Toepper, R., \& Thron, A. (2005). Effects of long-term practice and task complexity in musicians and nonmusicians performing simple and complex motor tasks: implications for cortical motor organization. Human Brain Mapping, 25, 345-352.

Miall, R.C. (2003). Connecting mirror neurons and forward models. Neuroreport, 14, 2135-2137.

Miyachi, S., Hikosaka, O., \& Lu, X. (2002). Differential activation of monkey striatal neurons in the early and late stages of procedural learning. Experimental Brain Research, 146, 122-126.

Molnar-Szakacs, I., Kaplan, J., Greenfield, P.M., \& Iacoboni, M. (2006). Observing complex action sequences: The role of the fronto-parietal mirror neuron system. NeuroImage, 33, 923-935.

Poldrack, R.A., Sabb, F.W., Foerde, K., Tom, S.M., Asarnow, R.F., Bookheimer, S.Y., $\&$ Knowlton, B.J. (2005). The neural correlates of motor skill automaticity. Journal of Neuroscience, 25, 5356-5364. 
Press, C., Bird, G., Flach, R., \& Heyes, C. (2005). Robotic movement elicits automatic imitation. Cognitive Brain Research, 25, 632-640.

Prinz, W. (2006). What re-enactment earns us. Cortex, 42, 515-517.

Rizzolatti, G., Fogassi, L., \& Gallese, V. (2001). Neurophysiological mechanisms underlying the understanding and imitation of action. Nature Reviews Neuroscience, 2, 661-670.

Rizzolatti, G., \& Craighero, L. (2004). The mirror-neuron system. Annual Review of Neuroscience, 27, 169-192.

Sakreida, K., Schubotz, R.I., Wolfensteller, U., \& von Cramon, D.Y. (2005). Motion class dependency in observers' motor areas revealed by functional magnetic resonance imaging. The Journal of Neuroscience, 25, 1335-1342.

Schubotz, R.I. (2007). Prediction of external events with our motor system: towards a new framework. Trends in Cognitive Sciences, 11, 211-218.

Umilta, M.A., Kohler, E., Gallese, V., Fogassi, L., Fadiga, L., Keysers, C., \& Rizzolatti, G. (2001). I know what you are doing: A neurophysiological study. Neuron, 31, 155165 .

van Mier, H. (2000). Human Learning. In A.W. Toga, \& J.C. Mazziotta (Eds.), Brain Mapping: The Systems (pp. 605-617), San Diego: Academic Press.

Wu, T., Kansaku, K., \& Hallett, M. (2004). How self-initiated memorized movements become automatic: a functional MRI study. Journal of Neurophysiology, 91, 16901698.

Zakzanis, K.K., Mraz, R., \& Graham, S.J. (2005). An fMRI study of the Trail Making Test. Neuropsychologia, 43, 1878-1886. 


\section{Summary}

The main goals of the research presented in this thesis were to deepen our understanding of how movement sequences are represented in the human brain, how these neural representations might change over the course of learning, and how information on the learned motor routines can be accessed in a different context once practice has ended. The described experiments were performed using functional Magnetic Resonance Imaging ( $\mathrm{AMRI}$ ), a non-invasive neuroimaging technique which allows to measure human brain activity in vivo and with great spatial detail, while subjects are actively engaged in a given task. The motor task which played a central role in the conducted experiments involved the learning of new movement sequences by tracing predefined 2dimensional maze trajectories. In contrast to the majority of AMRI studies in the motor domain, the chosen experimental paradigm required the execution of continuous movement sequences instead of discrete button presses. Furthermore, information on the correct trajectory could only be extracted from proprioceptive and somatosensory cues obtained during the actual execution of the movement sequence, since no visual guidance or feedback was provided.

To enable the registration of detailed behavioral data on the performed tracing movements while simultaneously collecting PMRI data on the associated brain activation patterns, a novel setup was developed as described in Chapter 2. The developed registration method made use of the fact that the mazes' geometries were predefined, constituting paths which could be represented by a onedimensional measure. The (changes in the) position of the used pen could be reconstructed by measuring the resistance between the current pen position and both ends of the maze path. This resistance-based reconstruction was performed with high spatial and temporal resolution, was independent of inevitable variations in the contact resistance between the pen and the resistive maze path, and was robust due to a redundant measurement scheme and built-in calibration features. Additionally, the setup was shown to be fully MR-compatible and provided extensive logging options to enable offline quality assurance and synchronization with the acquired fMRI data.

The resistive maze tracing setup was employed during the fMRI experiment reported in Chapter 3. In this study, subjects learned to perform a set of continuous movement sequences based on the experimental paradigm described above. By including two separate scanning sessions, the behavioral and neural manifestations of learning could be monitored at two different time scales: both within a time frame of several minutes (intra-session learning) and across several days (inter-session learning). Using a cortex-based alignment procedure for inter-subject normalization, a distributed network of bilaterally activated regions was identified during the performance of the movement sequences. 
Within this network, the activation in a set of premotor and parietal areas was shown to be specifically related to the ongoing learning process, independent of the potentially confounding effect of concomitant increases in movement velocity. Furthermore, the obtained behavioral data were used to individually determine two distinct stages (early vs. late) of the learning process in both sessions. Quite remarkably, consistent decreases in activation level throughout the network of learning-related areas could already be observed within the first scanning session, and were replicated four days later when either a completely novel maze or a previously encountered but not extensively trained maze was presented in the second scanning session. In contrast, no additional decreases in activation were found in the second scanning session for the sequence which had been extensively trained on the days between both measurements. Because the general layout of the involved network remained unchanged across different levels of proficiency, these data suggest that learning to execute the required movement sequences did not lead to a reorganization of functional activations but rather was accompanied by more efficient processing within the recruited regions as learning progressed.

The IMRI study described in Chapter 4 was conducted to investigate how information on the learned movement sequences could be accessed based on visual (i.e., non-motor) cues in the context of a matching task. During this experiment, dynamic visualizations of movement trajectories were presented which either did or did not match with one of the sequences the subjects had previously performed. Interestingly, when subjects were asked to discriminate between the matching and non-matching trajectories, the same brain areas which were involved in the actual learning of the movement sequences were reactivated, although no overt sequential movements were performed at that point and subjects had never seen what they had been doing before. This reactivation effect was robust in the sense that it was observed during two separate scanning sessions, indicating that a similar strategy to solve the task was used on both occasions. The reactivation of motor-related areas was interpreted as reflecting an internal simulation of the learned motor routines guided by the percept of their visual counterparts, and was shown to be independent of the button press which needed to be produced to indicate the classification of the stimuli. Furthermore, the strength of this reactivation was determined by the amount of previous experience with executing a specific sequence, as indicated by the finding that trajectories which could be performed most proficiently elicited the strongest responses. This experience-dependent modulation is in line with current theories which state that the meaning of an observed action can be extracted by an internal simulation of that action, a strategy which is most successful when the action is part of the observer's own motor repertoire. However, the reactivation of the complete learning-related network also suggests that if an internal simulation truly forms the mechanism 
through which the understanding of observed actions is achieved, a more widespread pattern of brain activity would be expected than is currently being assumed in the predominant theories on such phenomena.

Finally, the findings from the previous chapters are shortly summarized and discussed in Chapter 5. Besides providing a broader framework for the interpretation of the reported data and describing some of the performed studies' merits and shortcomings, potential future lines of research are presented in this chapter.

In sum, the work reported in this thesis demonstrates the feasibility of recording detailed behavioral data on continuous movement patterns in an fMRI setting, and illustrates how the obtained information can be successfully used to better interpret the accompanying imaging data at the single subject level. Furthermore, the presented results lend support to the concept of dynamic neural representations in motor learning in a twofold way. On the one hand, the neural representations of the studied movement sequences were shown to change systematically over the course of learning, even within a relatively short time window. On the other hand, the dynamic nature of the constructed representations was emphasized by the reactivation of the learning-related motor areas during a purely perceptual matching task. Instead of constituting a rigid and abstract reflection of what was learned, the neural representation of the stored motor sequences seemingly allowed a flexible internal re-enactment of the performed movements. Future efforts should be directed at further delineating the exact functional role of the nodes within the identified cortical network, and should incorporate more advanced analysis techniques (e.g., enabling the detection of transient responses or changes in connectivity patterns) to gain additional insights into the dynamic way in which the human brain represents information on voluntarily generated movement sequences. 


\section{Samenvatting}

De belangrijkste doelstellingen van het in dit proefschrift gepresenteerde onderzoek waren het verdiepen van onze kennis over hoe bewegingssequenties in het menselijke brein gerepresenteerd worden, hoe deze representaties veranderen gedurende het leertraject, en hoe informatie over reeds opgeslagen motorische routines opnieuw opgehaald kan worden in een andere context zodra het leerproces is beëindigd. Bij de beschreven experimenten werd gebruikt gemaakt van functional Magnetic Resonance Imaging (fMRI), een noninvasieve neuroimaging techniek waarmee de activiteit van het menselijk brein in vivo en met relatief hoge spatiele resolutie gemeten kan worden, terwijl een proefpersoon actief bezig is een willekeurige taak uit te voeren.

In de motorische taak die een centrale rol speelde in de uitgevoerde experimenten werden proefpersonen gevraagd nieuwe bewegingssequenties aan te leren door middel van het met een pen afleggen van vooraf gedefinieerde trajecten door 2-dimensionele doolhoven. In tegenstelling tot het grote merendeel van de eerder uitgevoerde $\mathrm{AMRI}$ studies, betrof het gekozen experimentele paradigma de uitvoering van continue bewegingssequenties in plaats van de discrete vinger bewegingen die normaalgesproken worden uitgevoerd bij het gebruik van een knoppenkast. Bovendien kon de benodigde informatie over het te volgen traject alleen aan hand van proprioceptische en somatosensorische cues gedurende de daadwerkelijke uitvoering van de bewegingen door de doolhoven achterhaald worden, omdat hierover verder geen enkele visuele feedback beschikbaar was.

Om gedetailleerde gedragsmaten met betrekking tot de afgelegde trajecten tegelijkertijd met de fMRI data over de daarbij optredende hersenactiviteit te kunnen meten, werd er een nieuwe meetopstelling ontwikkeld, zoals beschreven in Hoofdstuk 2. De ontwikkelde registratie methode maakt gebruik van het feit dat de af te leggen trajecten van te voren vastliggen (door de vorm van de verschillende doolhoven), en daardoor uiteindelijk door een 1-dimensionele maat gerepresenteerd kunnen worden. De veranderingen in de positie van de gebruikte pen kunnen daarom gereconstrueerd worden door het meten van de weerstand tussen een willekeurige pen positie en beide uiteinden van een doolhof. Deze reconstructie kon worden uitgevoerd met een hoge spatiele en temporele resolutie, was onafhankelijk van onvermijdelijke variaties in de contactweerstand tussen de pen en het onderliggende oppervlak, en bleek robust door een reeks van redundante metingen en ingebouwde calibratie mogelijkheden. Daarnaast werd gedemonstreerd dat de meetopstelling volledig MR-compatibel is en uitgebreide log-opties bezit die het mogelijk maken om offline de gedragsdata te synchroniseren met de fMRI metingen en de kwaliteit van de opgeslagen data te controleren. 
De op weerstandsmetingen gebaseerde meetopstelling werd vervolgens gebruikt tijdens het fMRI experiment dat wordt beschreven in Hoofdstuk 3. In dit experiment werden proefpersonen gevraagd een aantal nieuwe bewegingssequenties te leren op de manier die in de vorige alinea's is geïntroduceerd. Door twee afzonderlijke scan sessies in het experiment op te nemen konden de gedragsmatige en neurale manifestaties van leren gevolgd worden gedurende verschillende fases: allereerst binnen het tijdsbestek van enkele minuten, maar ook over een periode van meerdere dagen. De resultaten lieten zien dat een gedistribueerd netwerk van bilateraal oplichtende hersengebieden betrokken was bij het leren van de vereiste bewegingen. De gemeten activiteit binnen een set van premotorische en parietale gebieden kon specifiek gerelateerd worden aan het zich voltrekkende leerproces, los van mogelijk storende effecten die veroorzaakt zouden kunnen worden door het tegelijkertijd toenemen van de snelheid waarmee de bewegingen werden uitgevoerd. Bovendien werden voor beide scan sessies en voor elke proefpersoon afzonderlijk twee fases binnen het leerproces onderscheiden (vroeg vs. laat) aan hand van de gemeten gedragsdata. Hierdoor konden al tijdens de eerste scan sessie specifiek aan leren gerelateerde en consistente dalingen in activatie aangetoond worden voor de reeds geïdentificeerde hersengebieden, die vier dagen later in de tweede scan sessie bevestigd werden wanneer een compleet nieuw doolhof geleerd moest worden, of verder gegaan werd met het oefenen van een reeds eerder uitgevoerde sequentie. Tijdens het uitvoeren van de intussen geautomatiseerde bewegingssequentie (die de proefpersonen uitvoerig hadden geoefend gedurende de dagen tussen beide scan sessies) werden daarentegen echter geen verdere dalingen in activiteit gevonden. Omdat de constellatie van het corticale netwerk dat betrokken was bij de uitvoering van de vereiste bewegingen vrijwel ongewijzigd bleef, lijken deze data te suggereren dat het bestudeerde leerproces voornamelijk leidt tot een efficiëntere verwerking binnen een consistent geactiveerd neuraal netwerk, in plaats van eerder in de literatuur voorgestelde functionele reorganisaties waarbij verschillende hersengebieden betrokken zijn al naar gelang in welke leerfase de proefpersoon zich bevindt.

De $\mathrm{AMRI}$ studie uit Hoofdstuk 4 werd uitgevoerd om na te gaan hoe op hersenniveau toegang verkregen kan worden tot informatie over de aangeleerde sequenties van bewegingen, gebaseerd op visuele (i.e., non-motorische) cues in de context van een zogenaamde matching taak. In dit experiment werden dynamische visualisaties van verschillende bewegingstrajecten aan de proefpersonen aangeboden, die òf overeenkwamen, òf juist afweken van de bewegingen die de proefpersonen eerder al zelf hadden uitgevoerd. Uit de resultaten bleek dat dezelfde hersengebieden die eerder betrokken waren bij het aanleren van de daadwerkelijke bewegingen, nu gebruikt werden om deze verschillende stimulus klassen van elkaar te onderscheiden, ondanks dat er op 
dit moment geen sequentiële beweging uitgevoerd werd en proefpersonen nooit eerder hadden gezien wat ze deden. Dit reactivatie effect was robust in de zin dat het afzonderlijk in beide scan sessies kon worden waargenomen, wat erop duidt dat de taak in beide gevallen op eenzelfde wijze werd uitgevoerd. Deze reactivatie van de hersengebieden die oorspronkelijk betrokken waren bij het leren van de bewegingen zelf werd gezien als een aanwijzing voor een intern simulatie proces waarbij de neurale representaties van de eerder uitgevoerde bewegingen opnieuw geactiveerd werden door het waarnemen van de overeenkomstige visuele weergave. Bovendien werd aangetoond dat de gevonden reactivatie niet te wijten zou kunnen zijn aan de beweging die proefpersonen uitvoerden om hun classificatie (match of non-match) van de gepresenteerde stimuli duidelijk te maken. Tot slot werd ook nog vastgesteld dat de mate van reactivatie afhankelijk was van de hoeveelheid training die eerder was geïnvesteerd, doordat de trajecten die overeenkwamen met de meest intensief geoefende beweging ook de sterkste respons in de hersenen teweeg brachten. Het optreden van deze aan ervaring gerelateerde modulatie is in overeenstemming met recente theorieën waarin gesteld wordt dat de betekenis van geobserveerde acties door een interne simulatie achterhaald kan worden, en dat dit mechanisme het meest effectief is als de geobserveerde beweging overeenkomt met een beweging die deel uit maakt van het gedragsrepertoire van de toeschouwer. Aan de andere kant suggereren de verzamelde data echter ook dat een meer uitgestrekt netwerk van corticale hersengebieden hierbij betrokken is dan op dit moment in de meest gangbare theorieën wordt aangenomen.

In Hoofdstuk 5 ten slotte, worden de bevindingen uit de eerdere hoofdstukken nog eens kort samengevat en besproken. Hierbij worden naast enkele positieve aspecten en tekortkomingen van de uitgevoerde studies ook een aantal suggesties gedaan voor vervolgonderzoek, en worden de huidige resultaten in een bredere context uiteengezet.

Samenvattend kan gesteld worden dat de studies die in dit proefschrift zijn opgenomen laten zien dat het mogelijk is om gedetailleerde gedragsdata over continue bewegingssequenties te verzamelen in een fMRI omgeving, en illustreren dat de zo verkregen informatie op een succesvolle wijze toegepast kan worden om de bijbehorende fMRI data beter te kunnen interpreteren. Bovendien werd er op twee manieren verdere ondersteuning gevonden voor het concept van dynamische neurale representaties in de context van motorisch leren. Enerzijds werd aangetoond dat de neurale representaties van de bestudeerde bewegingen systematische veranderingen ondergingen gedurende het leerproces, zelfs binnen een vrij kort tijdsbestek. Anderzijds werd het dynamische karakter van de geconstrueerde representaties benadrukt doordat de hersengebieden die betrokken waren bij dit leerproces opnieuw geactiveerd 
werden tijdens een volledig op visuele informatie berustende matching taak. Dit geeft aan dat de neurale representaties van de opgeslagen bewegingsroutines geenszins een rigide en slechts abstracte reflectie van het geleerde vormen, maar blijkbaar flexibel ingezet kunnen worden om een interne simulatie van de eerder uitgevoerde bewegingen te verwezenlijken. Verder onderzoek zou vooral gericht moeten zijn op het duidelijker definiëren van de functie die de hier gerapporteerde hersengebieden afzonderlijk vervullen, en zou gebruik moeten maken van meer geavanceerde analyse methoden (b.v. om ook snelle, kort durende responsen of veranderingen in connectiviteits-patronen te kunnen detecteren) om zo nog meer inzicht te krijgen in de dynamische wijze waarop het menselijke brein informatie over willekeurig gegenereerde bewegingen representeert. 
Acknowledgements

\section{Acknowledgements}

Progress can be described as a succession of interrelated crises. At certain points in time, you will therefore inevitably feel lost in the maze which every scientific endeavor metaphorically constitutes. To eventually find your way in spite of any encountered hardships, it is essential to be able to rely on people who take you by the hand, either to guide you on or simply accompany you along the way. These are the people who I would like to thank here.

First of all, I'm grateful to my supervisors Hanneke van Mier and Rainer Goebel. Hanneke, besides granting me the opportunity to start this PhD-project in the first place, you (almost) unconditionally supported my (often timeconsuming) efforts, no matter how many additional calibration measurements I thought were necessary or how fast important deadlines were approaching. Your door was always open, and I've greatly appreciated your input. Rainer, your widely acknowledged enthusiasm (more full professors should add "really cool" to their vocabulary) and almost visionary scientific point of view are truly inspiring. Your contributions (in the form of 'life-saving' QX executables compiled in India and otherwise) have been invaluable and I'm looking forward to our continuing collaboration.

Bettina, it's great to have somebody around who wholeheartedly agrees that seemingly inconsequential details often do matter in the end. Amongst other things, you taught me that creating segmentations is an art, and I'm glad to have you around as a colleague and friend. Amanda, thank you for your sincere interest in my projects (on a professional as well as personal level), and all your cordial support and advice over the years. I do miss our occasional 'hallwaybrainstorms'.

I would also like to thank my former and present roomies: Jens (finishing that first 'co-authored' m-file was a real turning point), Vincent (thanks for the musical references; the chances that we might actually start this little project of ours do not seem too bad I'd say), Lourens (statistical benefactor and instigator of political discussions) and Francesco (our very own personification of Italian élan).

Furthermore, numerous colleagues/friends from Brain Innovation, the illustrious Vision Group, and the Cognitive Neuroscience department in general have contributed to a nice and creative atmosphere in which working hard and feeling at ease are by no means two mutually exclusive states. Although a general 'you know who you are' would apply here, I at least would like to specifically thank the following people for their contributions to the projects described in this thesis: Armin (for additional info on everything which is not explicitly stated in the BrainVoyager user guide), Bernadette (for somehow being there at crucial stages), Elia (for filter tweaking, general motivation and 
shared reluctances), Peter (for helpful comments at the right times), and both Annemie and Riny (for skillfully compensating some of my organizational flaws). I am also much obliged to all the subjects who participated in the described experiments.

In addition, I am still very grateful to all the people who helped during the development and fabrication of the MR-compatible maze tracing setup: Günther Hilgers and the other members of the RWTH Aachen University who kindly agreed to participate, Erik and Paul from the FC Donders Centre in Nijmegen, Erik from Siemens, and Huub together with all the other members of our faculty's technical staff (for tolerating me during my unofficial 'internship' at their department).

Although being involved in neuroscientific research can be fascinating and rewarding, it also requires a considerable amount of dedication and can be accompanied by a certain degree of so-called tunnel vision. Therefore, I would like to thank the following people for reminding me that life has so much more to offer. Foremost, my sincere gratitude goes out to Har, Renée, Eric, Miranda, Leon, Robert, Angelo and Wouter, who all have shaped me in one way or another. Although we don't get the chance to hang out together too often, I am convinced that the reassurance which comes with knowing you're there should not be underestimated. Similarly, I still cherish the weekends together with the friends I met while studying in Maastricht, of which I would like to thank Véronique and Marleen in particular (amongst other things for still believing in certain questionable songs which already came up in too many conversations).

It is also comforting to realize that I could count on my parents, my siblings and their partners, and the rest of the family for continuous support and compassion, things which I hopefully will never take for granted. Although I have numerous reasons to thank you all, in the context of this thesis I would particularly like to express my gratitude to my mother (for your down to earth attitude which makes it easier to put things into perspective), my father (for your invaluable help and insight, your curiosity and drive, your anecdotes and optimism, and of course all the weekends I claimed during our collaboration), my sister Dorette (for the shown interest and proofreading parts of the thesis), my brother Mirko (for the cover illustration(s), your CWS-inspired view on neuroscience, and the occasionally offered escapes from my daily routines), and my godmother Dorothé (for keeping me up to date on neuroscience issues covered in the media).

Finally, there is one more person left to thank. Judith, you firmly held my hand when I needed it the most and guided me on whenever the path was unclear...thank you for everything...I look forward to continue our journey, with my hand in yours, never letting go... 


\section{Curriculum vitae}

Joel Reithler was born on May $2^{\text {nd }} 1977$ in Heerlen, The Netherlands. In 1995 he completed his secondary school education at the Scholengemeenschap Sophianum in Gulpen, after which he started his Psychology study at Maastricht University. Following an internship at the University Hospital of Maastricht's department of Psychiatry \& Neuropsychology, he became a research assistant at the Psychology faculty's Cognitive Neuroscience department in 1999, and obtained his master's degree in Biological Psychology one year later. Subsequently, he worked on his $\mathrm{PhD}$-project from 2001-2006 at the Cognitive Neuroscience department in Maastricht, where he currently continues his work as a postdoctoral fellow. 